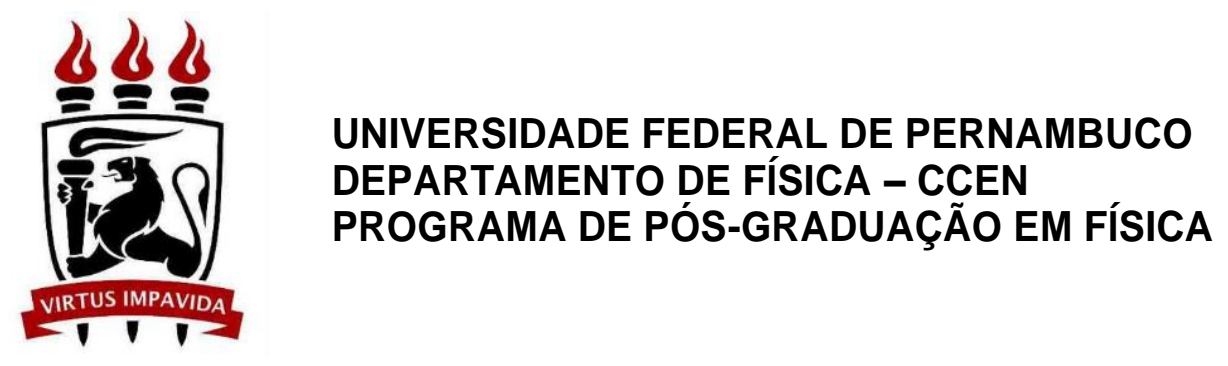

ARIDAY SAMIT MOSQUERA POLO

NONEQUILIBRIUM INTERBAND PHASE TEXTURES INDUCED BY VORTEX SPLITTING IN TWO-BAND SUPERCONDUCTORS 


\title{
NONEQUILIBRIUM INTERBAND PHASE TEXTURES INDUCED BY VORTEX SPLITTING IN TWO-BAND SUPERCONDUCTORS
}

\begin{abstract}
Tese apresentada ao Programa de Pós-Graduação em Física da Universidade Federal de Pernambuco, como requisito parcial para a obtenção do título de Doutor em Física.
\end{abstract}

Orientador:

Prof. Dr. José Albino Oliveira de Aguiar

Universidade Federal de Pernambuco 
Catalogação na fonte

Bibliotecário Jefferson Luiz Alves Nazareno CRB 4-1758

M912n Mosquera Polo, Ariday Samit.

Nonequilibrium interband phase textures induced by vortex splitting in two-band superconductors / Ariday Samit Mosquera Polo. - 2017.

101f.: fig., tab.

Orientador: José Albino Oliveira de Aguiar.

Tese (Doutorado) - Universidade Federal de Pernambuco. CCEN. Física, Recife, 2017.

Inclui referências.

1. Física da matéria condensada. 2. Supercondutividade. 3. Dinâmica de vórtices. I. Aguiar, José Albino Oliveira. (Orientador). II. Titulo. 


\section{NONEQUILIBRIUM INTERBAND PHASE TEXTURES INDUCED BY VORTEX SPLITTING IN TWO-BAND SUPERCONDUCTORS}

Tese apresentada ao Programa de Pós-Graduação em Física da Universidade Federal de Pernambuco, como requisito parcial para a obtenção do título de Doutor em Física.

Aprovada em: 20/10/2017.

\section{BANCA EXAMINADORA}

Prof. Dr. José Albino Oliveira de Aguiar Orientador

Universidade Federal de Pernambuco

Prof. Dr. Ernesto Carneiro Pessoa Raposo

Examinador Interno

Universidade Federal de Pernambuco

Prof. Dr. Maurício Domingues Coutinho Filho

Examinador Interno

Universidade Federal de Pernambuco

Prof. Dr. Andrey Chaves

Examinador Externo

Universidade Federal do Ceará

Prof. Dr. Mauro Melchiades Doria

Examinador Externo

Universidade Federal do Rio de Janeiro 
My family, my girlfrend Leidy Laura Diaz Dominguez and my cat Fliki 


\section{Acknowledgements}

Thank God for everything that allows us. Many people have helped and encouraged me throughout my candidature and I am deeply grateful to them. The guidance of my supervisor, José Albino Oliveira de Aguiar, has been invaluable. He has always been available to discuss in detail all of the problems, small and large, that I have faced along the way. Many thanks to Rogério Mendes da Silva for all his knowledge contributed. The collaboration of Arkady Shanenko and Alexei Vagov was very useful in the success of this project. 


\section{Resumo}

O modelo de Ginzburg-Landau com duas componentes foi aplicado a uma placa supercondutora com dois parâmetros de ordem, submetida a um campo magnético estático $(\mathbf{H})$ e a uma densidade de corrente $(\mathbf{j})$, para estudar as configurações e a dinâmica de vórtices. É demonstrado que, no limite de acoplamento fraco entre bandas, a interação entre vórtices penetrando na amostra com a superfície da mesma produz um fenômeno de separação de vórtices, dividindo os vórtices compósitos e criando vórtices fracionários. A interação entre vórtices, que é atrativa interbandas e repulsiva intrabanda e é controlada pela densida de corrente aplicada ao sistema, produz um ordenamento alternado de vórtices. Esse ordenamento cria texturas de fase de não-equilíbrio, ou domínios com sinais opostos da interação Josephson entre os condensados. Essa textura de fase possui efeitos significativos na dissipação causada pelo movimento de vórtices. Em particular, no regime de textura de fase, a corrente crítica, necessária para o inicio da dissipação, é deslocada para valores mais altos. É demonstrado também, que o aumento das dimensões da amostra dificulta o surgimento de vórtices separados, e que a introdução de barreiras lineares de ancoragem facilita o desacoplamento da rede de vórtices.

palavras-chave: Modelo de Ginzburg-Landau com duas componentes. Vórtices não compósitos. Barreiras lineares de ancoragem. 


\begin{abstract}
We demonstrate that in a weak-coupled two-band superconducting slab the interaction between vortices penetrating the sample and its boundaries leads to the phenomenon of vortex splitting, which divides composite vortices and creates fractional ones. The interaction between vortices, attractive for different bands and repulsive for the same band, which is controlled by the electric current density flowing through the system, leads to an ordered alternating arrangement of the vortices. This arrangement creates non-equilibrium interband phase textures or domains with different signs of the Josephson energy of the interaction between the band condensates. Such phase textures have a significant effect on the dissipation caused by the vortex motion. In particular, in the phase-texture regime the onset of the dissipation is shifted to higher current densities. It is also demonstrated that increasing the sample size hinders the emergence of separate vortices, and that the introduction of linear pinning barriers facilitates the decoupling of the vortex network.
\end{abstract}

Keywords: Two Component Ginzburg-Landau model. Non-composite vortices. Linear pinning barrier. 


\section{List of Figures}

1 A magnetic field $\mathbf{B}_{a}$ applied parallel to a plane vacuum-superconductor interface. The magnetic field decays to $\mathbf{B}_{a} / e$ at a distance $x=\lambda_{L}$ in the superconducting region. . . . . . . . . . . . . . . 2 20

2 A schematic picture of magnetization curves for bulk type $I$ and type $I I$ superconductors. For type $I$ the phase transition between the superconducting and normal states is of first order. We can observe a discontinuity between both states for a magnetic field $H_{c}$. For type $I I$ superconductor, the phase transition between both states is of second order and there is no discontinuity at a given $H_{c}$, instead, the superconductor enters a mixed

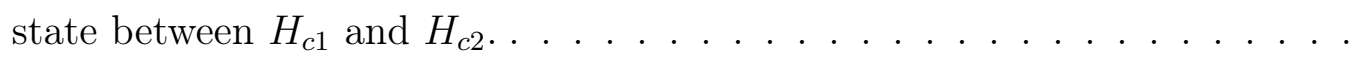

3 Configuration of the simulation. The current density $\mathbf{j}$ flows in at righ-hand side boundary and out at the left-hand side boundary of square superconductor. External fields $H_{+}$and $H_{-}$are applied outside the lower and upper boundaries, respectively. Pinning centers, where superconductivity is suppressed are represented by small squares. Taken from Ref. [1]. . . . . . . . 3

4 (a) A superconducting stripe of width $W$ with a central, time-dependent, pinning line of width comparable to the vortex size, and a 4-point probe applied $d c$ current (with density $J$ ), and measured voltage ( $V$ at contacts separated by distance $L$ ). A depletion region is simulated as sinusoidally oscillating local heating up to the critical temperature $T_{c}$ and back to working temperature, with frequency $\omega$. (b) Possible experimental setup corresponding to (a), by using laser light at the far field region passing through a metallic mask to create time-dependent and spatially modulated depletion of the superconducting condensate. Taken from Ref. [2]. . . . . . . . . . . 32

5 The dependence of the electric field (a) and of the velocities $v_{1}, v_{2}$ (b)on the current density $J \ldots \ldots \ldots \ldots$. . . . . . . . . . . . . . . 44

6 Sketch of cells defining the numbering of discrete variables. . . . . . . . . 46

$7 \quad$ Superconducting slab with infinite dimensions in $z$ and $x$ axis and finite in $y$ direction. A magnetic field $(H)$ is applied parallel at $z$ and current $(I)$

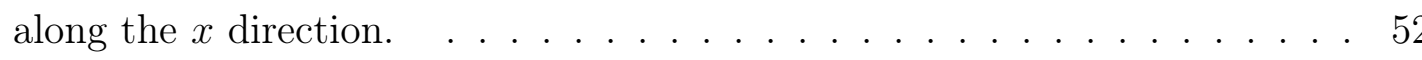


8 (a) Phase diagram $(\mathbf{H}-\mathbf{j})$ of vortex lattice states for $W=150, L=300$. The state of dissociated vortex is represented by phase $I I I$. (b)-(n) Vortex configurations corresponding to selected states $b, c, d, e, f, g, h, i, j, k, m$ and $n$, respectively from (a). The vortices in the first (second) band are represented by black (magenta) balls. The three vertical green dotted lines correspond to the fields, $H=0.24 H_{c}, H=0.73 H_{c}$ and $H=1.13 H_{c}$ respectively. For $H>H_{p}$ (and not comparable with $H_{p} \sim 0.65 H_{c}$ ) the phase of non-composite vortex (phase $I I I$ ) is absent, since the second band reaches the normal state before the composite vortices are dissociated, whereas the

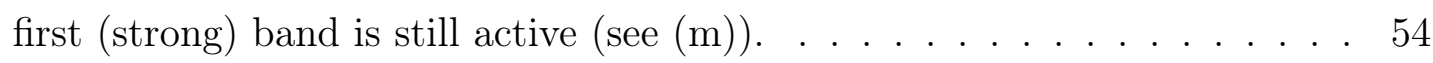

9 Ratio of the normal state to total area as a function of current for a) $H=0.24 H_{c}$ b) $H=0.73 H_{c}$ and c) $H=1.13 H_{c} \ldots \ldots \ldots$. . . . . . 57

10 The dependence of velocities of first (red) and second (blue) bands on the current density $j$ for $H=0.24 H_{c}$. These are the average velocities in the lower half of the slab. . . . . . . . . . . . . . . . . . . 58

11 The dependence of velocities of first (red) and second (blue) bands on the current $j$ for $H=0.73 H_{c}$. These are the average velocities in the lower half of the slab.

12 The dependence of velocities of first (red) and second (blue) bands on the current $j$ for $H=0.73 H_{c}$. These are the average velocities in the upper half of the slab.

13 Voltage (black) and correlation (blue) curves in function of current for $H=0,73 H_{c}, W=150, L=300$

14 a) Dissociation of vortex lattices in the instability point $p^{*}$ in Fig. 13 . b) The vortices reach the composite vortex state newly (point $p$ in Fig. 13) after the instability in $p^{*}$. $W=150, L=300$.

15 One possible scheme of non-composite vortex. The red vortex is in the first band and the red arrow indicating the direction to increase $\Psi_{1}$. The blue vortex is in the second band and the blue arrow indicating the direction to increase $\Psi_{2}$. Then $r$ is calculated in a direction that $\Psi_{1}$ increase and $\Psi_{2}$ decrease, so $r<0 . \ldots \ldots \ldots \ldots \ldots$

16 Voltage (black) and correlation (blue) curves for $H=0.24 H_{c}, W=150$, $L=300$ 
17 a) Vorticity of the first band $L_{1}$ (violet) and correlation $r$ (blue) in function of current density $j$ for the slab with $L=300, W=150$ and at $H=$ $0.73 \mathrm{Hc}$. The solid green lines separate the phases $I, I I$ and $I V$ explain in section 4.3. The vorticity curve (purple) is approximated by several linear behavior of different slopes (red, orange, yellow, and green lines). The magenta vertical lines point at particular sudden decreasing of $r(j)$ curve (blue) accompanied by high local changes (red lines) of vorticity curve (purple) . b)-e) Vortex configuration of states $b, c, d$ and $e$ selected respectively in a). The black (magenta) balls represent the vortices in the first (second) bands. The red arrows point to deformed vortices. . . . . . .

18 (a) Dependence of the correlation $(r)$ on the current density (j) for different strength of Josephson coupling $\lambda_{12}$ values. $\lambda_{12}=0.0001$ (black curve), $\lambda_{12}=0.0002$ (red), $\lambda_{12}=0.0005$ (blue), $\lambda_{12}=0.001$ (olive), $\lambda_{12}=0.001$ (orange). The phase $I I I$ is between two green dash lines and in this phase the correlation $r(\mathbf{j})$ increase with $\lambda_{12}$ increasing. (b)-(e) Vortex configurations corresponding to selected states $b, c, d$ and $e$, respectively from (a). The black (magenta) balls represent the vortices in the first (second) bands. The dimensions of slab and applied magnetic field are $W=300, L=150$ and $H=0.73 H_{c}$ respectively . . . . . . . . . . . . . . .

$19 \mathrm{~V}(\mathrm{j})$ curve for $H=0.73 H_{c}, L=300$ and $W=150$. In phase $I I I$ the curve present a non-linear increase due to the decoupling lattice and the LO instability. In phase $I V$ LO instability is the unique responsible for the non-linear increase. . . . . . . . . . . . . . . . .

$20 \mathrm{~V}(\mathrm{j})$ curve for $H=0,73 H_{c}, L=300$ and $W=150$. In phase $I I I$ the curve presents a non-linear behavior due to a strong influence of the LO instability ans small contribution of the decoupling lattice. . . . . . . . . . 67

$21 \mathrm{~V}(\mathrm{j})$ curve for $H=1,13 H_{c}, L=300$ and $W=150 \ldots \ldots \ldots$

22 Snapshots of the spatial distribution of the Josephson energy $g_{J}$, plotted as colour density plots, as defined in Eq. (2.9.1), calculated for the slab with the crossection $600 \times 150$ (in the $\xi_{1}$ units). Domains of $g_{J}<0$ and $g_{J}>0$ are marked by red and blue, respectively, colour intensity gives the absolute value $\left|g_{J}\right|$, white colour corresponds to $g_{J}=0$. Panels a), b), c), d) and e) are calculated, respectively, for the current $j=0.33,0.37,0.47,0.57,0.77 j_{0}$. Parameters of the system are discussed in the text. . . . . . . . . . . 72

23 Enlarged parts of the colour density plots of $g_{J}$ shown in Fig. 22, superimposed with the contour plots for the band condensate densities for the strong band $\left|\Psi_{1}\right|$ (black line) and for the weak band $\left|\Psi_{2}\right|$ (magenta line), which reflects the vortex structure in the band condensates. . . . . . . . . 
24 Dynamics of phase textures for a constant current $j=0.37 j_{0}$. The position of phase textures in four different and progressing times, $t=0,400,800$ and $1600 t_{0}$, reveal that this domains move toward the lower boundary $(\mathrm{y}=0)$.

25 Changes of phase textures for a settled current $j=0.47 j_{0}$. The spacial distribution of phase textures in four different and progressing times, $t=0,400,800$ and $1600 t_{0}$, reveal that the channels are almost static, but between them, some small phase textures regions travel from one edge to the other.

26 Snapshots of the Josephson energy distributions calculated for the slabs with $L \times w=600 \xi_{1} \times 100 \xi_{1}$ (panel a) and with $L \times w=600 \xi_{1} \times 300 \xi_{1}$ (panel b). The contours represent the condensate density $\left|\psi_{1}\right|$ (black lines) and $\left|\psi_{2}\right|$ (magenta lines). The current is $j=0.447 j_{0}$, other parameters are the same as in Fig. 22. . . . . . . . . . . . . . . . . . . . . 77

27 (Color online) Total length of the domain wall normalized by $w, L_{d w} / w$ as function of $j$ for some chosen values of $w$ and $H$. $L_{d w} / w$ measures the extent of vortex dissociation in the two-band superconducting sample because fractional vortices appear attached to such domain walls. . . . . . 78

$28 \mathrm{I}-\mathrm{V}$ characteristic for the set of the interband coupling $\lambda_{12}=0,0.0001,0.001$, and 0.005. Points a-e on the curve for $\lambda_{12}=0.0001$ correspond to Fig. 1 a) - e) and Fig. 2 a) - c), respectively. Notice that the I - V characteristic for $\lambda_{12}=0$, and 0.0001 are almost the same. . . . . . . . . . . . 78 
29 (a) Correlation, voltage and vorticity for the first band (stronger band) curves as a function of the applied current density, for a pinning free twoband superconducting slab, with $H=0.73 H_{c}, W=300$ and $L=300$. The solid green lines separate the phases $I, I I$ and $I V$ explain in section 4.3. The behavior of vorticity curve (purple) is approximated by four linear behavior (red, yellow, orange and dark cyan lines). Among this lines, the yellow line has the higher slope, which correspond with a particular decrease of $r(j)$, enclosed between magenta dotted lines (this is agree that we exposed in section 4.6). (b) $m^{*}$ state in $r(j)$ curve from (a): Domains of $g_{J}<0$ and $g_{J}>0$ are marked by red and blue, respectively. White colour corresponds to $g_{J}=0$. Here, $g_{J}$ is the Josephson energy defined in Eq. (2.9.1). The vortices in first (black line) and second (magenta line) bands are plotted. The domain walls do not cross the sample and the vortices are dissociated only in small regions of the sample. In the central region of slab the vortices are composite (c) $n^{*}$ state in $r(j)$ curve from (a): Although the current density of this state is higher than the current density of state $m^{*}$, there is a few dissociated vortices and the normal state in weaker band is more advanced. . . . . . . . . . . . . . . . . . . 82

30 Domain wall length as a function of the applied current density, for a free pinning system, with $H=0.73, W=300$ and $L=300$. . . . . . . . . . . 83

31 System with $W=300, L=300$ and $H=0.73 H_{c}$. (a) Length of domain walls for a free pinning system (black curve) and for a system with one linear barrier of length $D_{1}$ perpendicular to vortex move (red curve). (b) Length of domain walls for a systems with one (red curve) and two (olive curve) perpendicular linear barrier of length $D_{1}$. From (c) to (f):Domains of $g_{J}<0$ (red) and $g_{J}>0$ (blue). White colour corresponds to $g_{J}=0 . g_{J}$ is the Josephson energy defined in Eq. (2.9.1). The vortices in first (black line) and second (magenta line) bands are plotted. (c) Vortex configuration of state $m_{1}^{*}$ from $L(j)$ curve in (a). (d) Vortex configuration of state $n_{1}^{*}$ from $\mathrm{L}(\mathrm{j})$ curve in (a). (e) Vortex configuration of state $m_{2}^{*}$ from $\mathrm{L}(\mathrm{j})$ curve in (b). (f) Vortex configuration of state $n_{2}^{*}$ from L(j) curve in (b) . . . . . . . 85 
32 System with $W=300, L=300$ and $H=0.73 H_{c}(\mathrm{a})$ Length of domain walls for a systems with two (olive curve) perpendicular linear barrier of length $D_{1}$ and two parallel linear barrier of length $2 D_{1}$ (violet curve). (b) Domains of $g_{J}<0$ and $g_{J}>0$ are marked by red and blue, respectively. White colour corresponds to $g_{J}=0$. Here, $g_{J}$ is the Josephson energy defined in Eq. (2.9.1). The vortices in first (black line) and second (magenta line) bands are plotted. The domain walls cross the sample with some dissociate vortices attached them. Some vortices attached to domain walls are composite because the current is not sufficiently enough for dissociate them. (c) The domain walls cross the sample with dissociate vortices attached them. . . . . . . . . . . . . . . . . . . 87 


\section{Contents}

$\begin{array}{ll}\text { Introduction } & 15\end{array}$

State of art . . . . . . . . . . . . . . . 16

$1.2 \quad$ List of publications of this thesis . . . . . . . . . 18

Some topics of superconductivity $\quad 19$

London theory . . . . . . . . . . . . . . . . . 19

2.2 The Ginzburg-Landau equations . . . . . . . . . . . . 20

2.2.1 Boundary conditions . . . . . . . . . . . . 23

$2.3 \quad$ Type I and type II superconductors _ . . . . . . . . . . 23

$2.4 \quad$ Abrikosov vortice . . . . . . . . . . . . 24

2.4.1 Forces on Abrikosov vortice . . . . . . . . . . . . 25

2.4.2 Energy (E) of Abrikosov vortice . . . . . . . . . . . . . 25

2.4.3 Interaction energy of two Abrikosov vortices . . . . . . . . . . . 26

2.4.4 Quantization of magnetic flux and kinetic nature of driving force . 27

2.5 Flux pinning . . . . . . . . . . . . 30

$2.6 \quad$ Kosterlitz-Thouless transition . . . . . . . . . . . . 33

2.6.1 The two dimensional XY-model . . . . . . . . . . . 33

2.6.2 Vortex unbinding . . . . . . . . . . . 35

2.6.3 The spin wave stiffness . . . . . . . . . . . . . 35

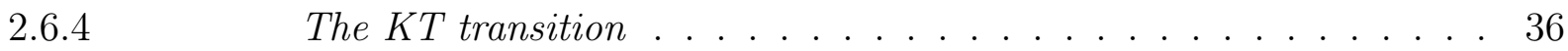

2.7 Multicomponent superconductivity based on multiband supercon-

ductors . . . . . . . . . . . . . . . . . 37

$2.8 \quad$ Two-component London superconductivity model . . . . . . . . . 39

2.9 Two-component Ginzburg-Landau (TCGL) model . . . . . . . . . . 39

$2.10 \quad$ Vortex dissociation in the London model . . . . . . . . . . . . 42

$\begin{array}{lll}3 & \text { Numerical method } & 45\end{array}$

3.1 $\psi$-U method . . . . . . . . . . . . . 45

3.1.1 Discretization of time dependent Ginzburg-Landau equations . . . . 45

3.1.2 Discretization of two-component Ginzburg-Landau (TCGL) equations 49

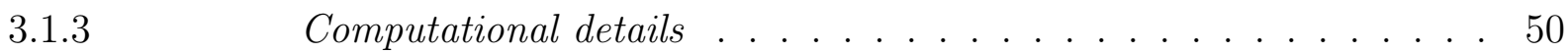


Introduction . . . . . . . . . . . . . . . . 51

Model and Method . . . . . . . . . . . . . . . . . . 52

Phases diagram $(\mathrm{H}-\mathrm{j})$ of vortex lattice configurations $\ldots . . . .53$

Interband vortex velocities . . . . . . . . . . . . . 57

Correlation as a measure to identify the non-composite vortex state 59

Rate of Entrying Vortices . . . . . . . . . . . . . . . . 62

The influence of Josephson coupling in the dissociation of vortices . 64

$\mathrm{I}-\mathrm{V}$ characteristics . . . . . . . . . . . . . . . 66

Summary . . . . . . . . . . . . . . . . . . . . 68

Interband phase textures induced by vortex splitting

Introduction . . . . . . . . . . . . . . . . . . . . 69

Model and Method . . . . . . . . . . . . . . . . . 70

Vortices near sample boundaries: general picture . . . . . . . . 71

Vortex dissociation and phase textures: numerical results . . . . 71

Dynamics of the phase textures . . . . . . . . . . . . . . 74

Size effects . . . . . . . . . . . . . . . . . . . 74

Current-voltage (I-V) characteristic . . . . . . . . . . . 76

Summary . . . . . . . . . . . . . . . . . . . . . 78

Creation of fractional vortices by linear pinning barriers $\quad 80$

Introduction . . . . . . . . . . . . . . . . . 80

Model and Method . . . . . . . . . . . . . . . . . . . . . 81

Two-band pinning free superconductor with large dimensions _ . . 81

Linear pinning barrier perpendicular to direction of vortex movement 83

Linear pinning barrier parallel to direction of vortex movement . . 86 


\section{Introduction}

Fundamental physical properties of superconductors were explained by the theory developed by John Bardeen, Leon Cooper, and Robert Schrieffer, the so called BCS theory of superconductivity [3]. The mean premise of this theory is that electrons near Fermi surface form Cooper pairs and condense into a macroscopic quantum state. John Bardeen, Leon Cooper and Robert Schrieffer received the Nobel Prize in 1972 for their achievment [3].

Many superconducting materials usually involve multiple Fermi surfaces. These superconductors can be described by a multiband BCS theory $[4,5]$, which was proposed shortly after the BCS theory. The discover of multiband superconductivity in $M g B_{2}$ [6] has attracted a lot of interest of the scientific community on multiband superconductors. Other materials of this type are the ferropnictides [7], heavy fermion compounds [8] and borocarbides [9].

The discovery of these types of superconductors brought a new dimension to the superconductivity research, revealing a variety of intriguing properties that are not found in their single-component counterparts. Among these properties we have the collective oscillation of numbers of Cooper pairs between different bands, known as the Leggett mode [10]. Multiband superconductors with interband Josephson coupling allow for the phase kink or phase soliton excitation due to the degenerate energy minima in the Josephson coupling. The phase soliton was first discussed by Tanaka in 2001 [11]. Other properties is the appearance of fractional vortices where vortex core can split between multiple bandspecific components of the superconducting condensate [12]. This type of vortices can be characterized by different winding numbers of the order parameter in the two superconducting components. Among such vortices one can distinguish a subclass of vortices when the phase winding exists only in one of the condensates. In general vortex in different condensates in the ground state is bounded and their normal cores are locked together to form a composite vortex.

It is an interesting question whether the composite vortex can dissociate. The answer to this question is the goal of this thesis. We present original results where the dissociation of composite vortices is analyzed as consequence of external magnetic field and external current density applied in a two component superconducting slab. The study is performed 
by using the two component Ginzburg Landau (TCGL) theory. We will analize two cases: A free pinning superconductor and a superconductor with a linear pinning barrier.

\subsection{State of art}

Now a days the research on multiband superconductors is inclined to discovery and explain its properties that are not shared by single-band superconductors. Among these properties, the behavior of a vortex is an interesting topological excitations for studying. When the phase difference between condensates change by $2 \pi$ inside superconductors, $a$ vortex excitation appears.

Alexei Abrikosov introduced the concepts of Abrikosov lattice [13] demonstrating that vortices in type II superconductor are arranged in a triangular lattice. After this discovery many works in single band superconductor have been published about the vortex dynamics in type II superconductors [14-21]. Vodolazov et al., [22] show that with increasing applied current, the moving vortex lattice changes its structure from a triangular one to a set of parallel vortex rows in a pinning free superconductor. Grimaldi et al., [23] elaborated a general phase diagram which includes all possible dynamic configurations of the Abrikosov lattice in a mesoscopic superconductor.

The calculation of interaction between vortices as a function of separation poses a challenge to theory since vortices are extended objects. Representative work in single band superconductor was realized by Jacob et al., [24] determining the interaction energy of two vortex configurations within the Ginzburg-Landau theory and showing that, when $\lambda / \xi<1 / \sqrt{2}$, the surface energy is positive and vortices attract each other (type $I$ superconductor). When $\lambda / \xi>1 / \sqrt{2}$, the surface energy is negative and vortices repel each other ( type $I I$ superconductor). Here $\lambda$ and $\xi$ are the penetration depth and coherence length respectively.

To date the understanding of competing vortex interactions in multiband superconductors has not been conclusively settled. In superconductors with multiple condensates the interaction between vortices present some interesting features. It is possible that vortices repel at short distant and attract at large separation [25]. Lin et al. [26] implemented and generalized the variational method for single-band superconductors [24] to calculate the inter-vortex interaction in a two-band superconductor.

The BCS correction to the Ginzburg-Landau theory for multiband superconductor lead to nonmonotonic vortex interaction and various works have been published in this matter. It was found in three-band superconductors with frustrated interband couplings [27]. The influence of the interband Josephson coupling was studied by Babaev et al. and [28] Chaves et al. [29] who studied the condition for the non-monotonic interaction.

The magnetic flux quantization in multiband superconductors deserves special attention. For a single band superconductor, the quantization of magnetic field is de- 
fined as follow: The magnetic flux $\Phi$ threading a superconducting loop or a hole in a bulk superconductor is quantized. It is a multiple integer of magnetic flux quantum $\Phi_{0}=h /(2 e) \approx 2.067833831(13) \times 10^{-15} \mathrm{~Wb}, \Phi=n \Phi_{0}$, with $n$ an integer, namely the winding number. Bogomolnyi [30] described the vortices carrying $n \Phi_{0}$ quantum flux with an integer $n>1$. These vortices with larger winding number $n$ are called giant vortices. The energy of this vortices is proportional to $n^{2}$ thus they are not energetically favorable in bulk superconductor, but in mesoscopic superconductors giant vortices may be stabilized by geometric confinement [31,32].

In multiband superconductors it is possible that the phase associated with the gap functions of condensates changes by different integer multiples of $2 \pi$ along a closed loop. Thus, the quantum flux associated with this vortex is not an integer multiple of $\Phi_{0}$. This type is called a fractional vortex. Using Ginzburg-Landau theory, Babaev et al. [12,33] demonstrated that in a two-band superconductor the flux is integer quantized $\Phi=n \Phi_{0}$ only when $n_{1}=n_{2}=n$. The other cases correspond with a fractional vortex and the most important are when $n_{1}=0, n_{2}=1$ or $n_{1}=1, n_{2}=0$ which are the cases with minimum energy. In bulk superconductors the fractional vortices are considered unstable because its energy diverges logarithmically with system size [34,35]. Therefore in the ground state these two fractional vortices lock together to form a composite vortex with equal winding number $n_{1}=n_{2}=1$. Rogerio et al. [36] showed that the resulting fractional vortex configurations leave distinct fingerprints in the static measurements of the magnetization, as well as in ac dynamic measurements of the magnetic susceptibility, both of which can be readily used for the detection of these fascinating vortex states in several existing multiband superconductors. Fractional vortices can also be stabilized in a mesoscopic two-band superconductor [37-40].

The dissociation of composite vortex lattice in the flux flow region due to the disparity of the vortex viscosity and flux of the vortex in different bands was predicted in [34]. This prediction was performed using the London free energy functional for two-band superconductors neglecting the Josephson interband coupling term. This dissociation has been discussed theoretically in multilayer superconductors too [41,42] and further observed experimentally $[43,44]$. The fractional vortices can be stabilized by pinning arrays [45] - When the external current is turned off suddenly in the decoupled phase where two fractional vortex lattices move with different velocities, it is possible that the fractional vortices get trapped by pinning centers if the density of pinning centers is higher than the vortex density.

In multicomponent superconductors, some superconducting states are degenerate in free energy. This leads to the appearance of a domain structure, this is, regions with different energies. Between these domains, domain walls appear as topological defects. When magnetic fields are applied to this domain structure, some of the vortices are trapped at the domain wall. The vortices at the domain wall form an exotic structure called 
a vortex sheet [46], where a conventional vortex splits into two vortices with half flux quanta $[12,47,48]$. Machida [1] investigated the flux flow and the pinning of the vortex sheet structure. He found that this moves with the flux flow. In the pinning case it is observed an emitting process of a conventional vortex from the vortex sheet by combining a pair of half flux-quantum vortices.

1.2 List of publications of this thesis

- Nonequilibrium interband phase textures induced by vortex splitting in two-band superconductors [49] . 


\section{Some topics of superconductivity}

\subsection{London theory}

The London theory was developed by the brothers Fritz and Heinz London. This phenomenological theory describe the specific electrodynamic properties of superconductors [50]. It is based on a two-fluid model [51]. We consider a superconductor composed of two current carrier (two-fluid) a normal and a superfluid one. The normal fluid carrier has density $n_{n}$, the superfluid carrier has density $n_{s}$, and the total fluid carrier density is $n=n_{n}+n_{s}$. The normal fluid obey the Ohm's law

$$
\mathbf{j}_{n}=\sigma_{n} \mathbf{E},
$$

with the normal conductivity $\sigma_{n}$ given by the Drude's law

$$
\sigma_{n}=\frac{e^{2} n_{n} \tau}{m}
$$

The supercurrent is given by

$$
\mathbf{j}_{s}=-e n_{s} \mathbf{v}_{s}
$$

with $\mathbf{v}_{s}$ the velocity of superfluid.

If we combine the Newton's equations

$$
\frac{d}{d t} \mathbf{v}_{s}=\frac{\mathbf{F}}{m}=-\frac{e \mathbf{E}}{m}
$$

with Eq. (2.1.3), we obtain

$$
\frac{\partial \mathbf{j}_{s}}{\partial t}=\frac{e \mathbf{v}_{s} n_{s}}{m} \mathbf{E}
$$

The Eq. (2.1.5) is the first London equation, which is valid only for $n_{n}$ and $n_{s}$ constants in the space and time. Taking curl of the 2.1.5 and integrating, we obtain the second London equation

$$
\nabla \times \mathbf{j}_{s}=-\frac{e^{2} n_{s}}{m c} \mathbf{B}
$$

By Ampere's law 


$$
\nabla \times \mathbf{B}=\frac{4 \pi}{c} \mathbf{j}_{s}+\frac{4 \pi}{c} \mathbf{j}_{n}
$$

Taking curl Eq. (2.1.7), using Eqs. (2.1.1) and (2.1.6), and employing some vectorial identities, finally we obtain

$$
\nabla^{2} \mathbf{B}=\frac{1}{\lambda_{L}^{2}} \mathbf{B}
$$

where $\lambda_{L}$ is known as London penetration depth

$$
\lambda_{L}=\sqrt{\frac{m c^{2}}{4 \pi e^{2} n_{s}}} .
$$

Let us consider a semi-infinite superconductor filling the half space $x>0$. If we apply a external magnetic field $\mathbf{B}_{a}$ parallel to the surface, the solution of Eq. 2.1.8 is

$$
\mathbf{B}(x)=\mathbf{B}_{a} e^{-x / \lambda_{L}} \text { for } x \geq 0
$$

The magnetic field thus decresases exponentially with the distance from the surface of the superconductor (Figure 1 ).

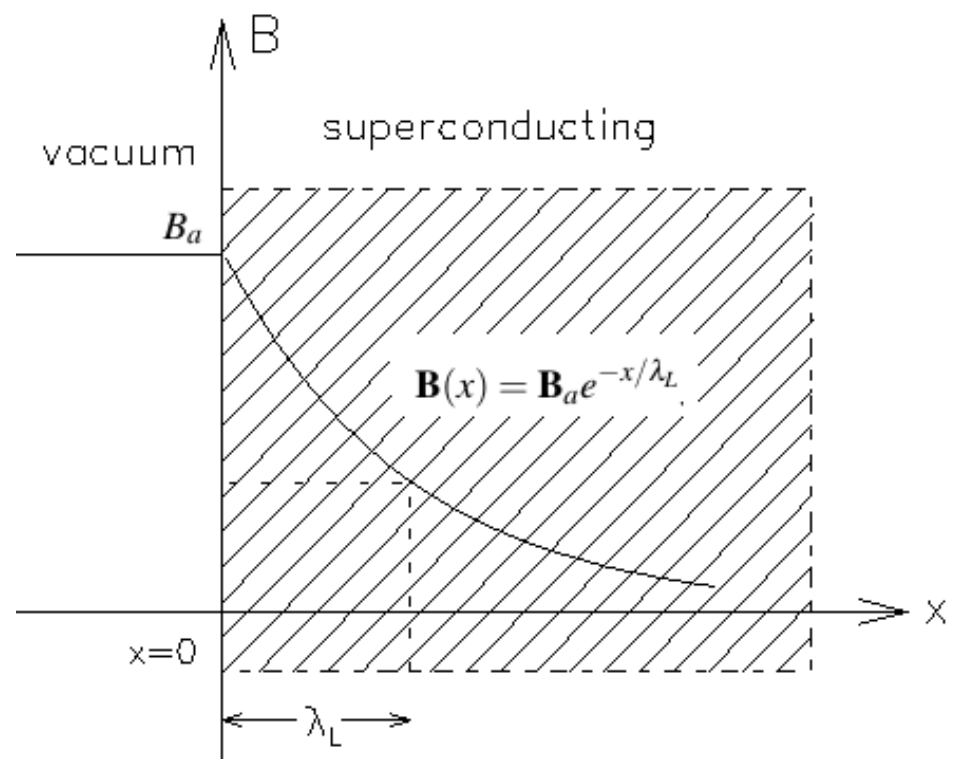

Figure 1: A magnetic field $\mathbf{B}_{a}$ applied parallel to a plane vacuum-superconductor interface. The magnetic field decays to $\mathbf{B}_{a} / e$ at a distance $x=\lambda_{L}$ in the superconducting region.

\subsection{The Ginzburg-Landau equations}

The complete fundamental equations for macroscopic superconductivity were written by Ginzburg and Landau in 1950 [52]. This classical mean field theory of continuous sec- 
ond order phase transitions introduce an order parameter that is zero above the phase transition and take values different of zero below of this transition. The phase transition occur at the critical temperature $T_{c}$. The order parameter is a complex function that conveniently is defined as

$$
\Psi(\mathbf{r})=|\Psi(\mathbf{r})| e^{i \theta(\mathbf{r})}=\sqrt{n_{s}(\mathbf{r})} e^{i \theta \mathbf{r}}
$$

where $n_{s}(\mathbf{r})$ is the superfluid density and $\theta$ is the phase that expresses the spontaneous breaking of the continuous (gauge) symmetry.

The Ginzburg-Landau (GL) total free energy has the form

$$
F_{t o t}(\Psi(\mathbf{r}), \mathbf{A}(\mathbf{r}))=\int d^{3} \mathbf{r} F_{G L}(\mathbf{r}),
$$

where $\mathbf{A}(\mathbf{r})$ is the vector potential and $F_{G L}(\mathbf{r})$ is the GL free energy density. Close to $T_{c}$ the order parameter is very small and the free energy density in the absence of an applied magnetic field can be expanded as follow

$$
F_{G L}=F_{N}+\alpha(T)|\Psi(\mathbf{r})|^{2}+\frac{1}{2} \beta|\Psi(\mathbf{r})|^{4}+\ldots
$$

$F_{N}$ is the density energy in the normal state and we have truncated the expansion at fourth order. For $T>T_{c}, \alpha>0$ and $\alpha<0$ for $T<T_{c}$, hence

$$
\alpha \cong \alpha\left(T-T_{c}\right)
$$

When the energy density of a magnetic field is accounted, the free energy density of the superconducting state in GL theory is

$$
F_{G L}=F_{N}+\frac{1}{2 m^{*}}\left|\left(-i \hbar \nabla+\frac{e^{*} \mathbf{A}(\mathbf{r})}{c} \Psi(\mathbf{r})\right)\right|^{2}+\alpha(T)|\Psi(\mathbf{r})|^{2}+\frac{1}{2} \beta|\Psi(\mathbf{r})|^{4}+\frac{\mathbf{B}(\mathbf{r})^{2}}{2 \pi} .
$$

$\mathbf{B}(\mathbf{r})$ is the magnetic induction and $m^{*}$ and $e^{*}$ are effective mass and charge of electron respectively.

Minimizing the free functional energy with respect $\Psi^{*}(\mathbf{r})$ and $\mathbf{A}(\mathbf{r})$, the GL equations are obtained

$$
\begin{gathered}
\frac{1}{2 m^{*}}\left(-i \hbar \nabla+\frac{e^{*} \mathbf{A}(\mathbf{r})}{c} \Psi(\mathbf{r})\right)^{2}+\alpha(T) \Psi(\mathbf{r})+\beta|\Psi(\mathbf{r})|^{2} \Psi(\mathbf{r})=0 \\
\frac{c}{4 \pi} \nabla \times \nabla \times \mathbf{A}(\mathbf{r})=\frac{i e^{*} \hbar}{2 m^{*}}\left(\Psi^{*}(\mathbf{r}) \nabla \Psi(\mathbf{r})-\Psi(\mathbf{r}) \nabla \Psi^{*}(\mathbf{r})\right)-\left.\frac{e^{* 2}}{m^{*} c} \Psi(\mathbf{r})\right|^{2} \mathbf{A}(\mathbf{r}) .
\end{gathered}
$$

Remembering the Ampere's law $\mathbf{j}(\mathbf{r})=\frac{c}{4 \pi} \nabla \times \nabla \times \mathbf{A}(\mathbf{r})$ the supercurrent can be identified as 


$$
\mathbf{j}(\mathbf{r})=\frac{i e^{*} \hbar}{2 m^{*}}\left(\Psi^{*}(\mathbf{r}) \nabla \Psi(\mathbf{r})-\Psi(\mathbf{r}) \nabla \Psi^{*}(\mathbf{r})\right)-\left.\frac{e^{* 2}}{m^{*} c} \Psi(\mathbf{r})\right|^{2} \mathbf{A}(\mathbf{r}) .
$$

In the limiting case of $\Psi(\mathbf{r}) \mid=$ const the Eq. (2.2.6) is reduced to the London equation Eq. (2.1.8). The GL theory has been applied to several critical phenomena [53,54], besides to the superconductivity.

For the case of zero applied field in Eq. (2.2.6) we obtain

$$
-\frac{\hbar^{2}}{2 m^{*}} \nabla^{2} \Psi(\mathbf{r})+\alpha(T) \Psi(\mathbf{r})+\beta|\Psi(\mathbf{r})|^{2} \Psi(\mathbf{r})=0
$$

Dividing the last equation by $\alpha(T)$, the gradient term becomes dimensionless and the quantity $\xi$ defined by

$$
\xi(T) \equiv\left(\frac{\hbar^{2}}{2 m^{*} \alpha(T)}\right)^{1 / 2}
$$

has dimension of a length. The quantity $\xi$ is called coherence length which determines the length over which the order parameter varies significantly.

Now we will find the characteristic length scale on which the magnetic field varies significantly. For this task we assume that the order parameter is uniform, so the gradient of $|\Psi(\mathbf{r})|$ in Eq. (2.2.7) can be neglected. We get the follow equation

$$
\frac{m^{*} c^{2} \beta}{4 \pi e^{* 2}|\alpha(T)|} \nabla \times \nabla \times \mathbf{A}(\mathbf{r})=-\mathbf{A}(\mathbf{r})
$$

and the penetration depth $\lambda$ is defined by

$$
\lambda(T) \equiv\left(\frac{m^{*} c^{2} \beta}{4 \pi e^{* 2}|\alpha(T)|}\right)^{1 / 2} .
$$

It is convenience to write the GL equations in dimensionless unit. For that we normalize the lengths by $\lambda$, the order parameter by $\left|\Psi_{0}(\mathbf{r})\right|=\sqrt{\frac{\beta}{|\alpha|}}$, the vector potential by $A_{0}=\frac{1}{\sqrt{2} H_{c} \lambda}$, where $H_{c}=\left(\frac{8 \pi \alpha^{2}}{2 \beta}\right)^{1 / 2}$ is the critical field. Finally we get the normalize GL equations

$$
\begin{gathered}
\left(-\frac{i}{k} \nabla+\mathbf{A}(\mathbf{r})\right)^{2} \Psi(\mathbf{r})-\Psi(\mathbf{r})+|\Psi(\mathbf{r})|^{2} \Psi(\mathbf{r})=0 \\
\nabla \times \nabla \times \mathbf{A}(\mathbf{r})+|\Psi(\mathbf{r})|^{2} \mathbf{A}(\mathbf{r})+\frac{i}{2 k}\left(\Psi^{*}(\mathbf{r}) \nabla \Psi(\mathbf{r})-\Psi(\mathbf{r}) \nabla \Psi^{*}(\mathbf{r})\right)=0
\end{gathered}
$$




$$
\mathbf{j}(\mathbf{r})=\frac{1}{2 i k}\left(\Psi^{*}(\mathbf{r}) \nabla \Psi(\mathbf{r})-\Psi(\mathbf{r}) \nabla \Psi^{*}(\mathbf{r})\right)-|\Psi(\mathbf{r})|^{2} \mathbf{A}(\mathbf{r})
$$

where $k=\frac{\lambda}{\xi}$ is defined as GL parameter.

\subsubsection{Boundary conditions}

\section{Boundary condition for the vector potential (A)}

For a superconductor of finite size a boundary condition for the vector potential requires that the magnetic field in the surface must be equal to external magnetic field applied $\left(\mathbf{H}_{a}\right)$.

$$
(\nabla \times \mathbf{A}) \times\left.\widehat{\mathbf{n}}\right|_{s}=\mathbf{H}_{a} \times\left.\widehat{\mathbf{n}}\right|_{s}
$$

where $\widehat{\mathbf{n}}$ is the unit normal vector of a surface.

\section{Boundary condition for the order parameter}

For the case of superconductor-vacuum surface

$$
\left.\widehat{\mathbf{n}} \cdot(-i \nabla-\mathbf{A}) \Psi\right|_{s}=0
$$

this implies that the normal current perpendicular to the boundary is zero.

A generalization of the condition was made by De Gennes [54]

$$
\left.\widehat{\mathbf{n}} \cdot(-i \nabla-\mathbf{A}) \Psi\right|_{s}=\left.\frac{i}{b} \Psi\right|_{s}
$$

- For superconductor-vacuum or superconductor surfaces $b \rightarrow \infty$.

- When the interface is superconductor-metal, $b>0$. This implies that the superconductivity is suppressed at the edges of the sample.

- If the interface is superconductor to superconductor of higher $T_{c}, b<0$. In this case the superconductivity is increased in the boundaries.

\subsection{Type I and type II superconductors}

Let us consider a superconductor in a presence of external magnetic field. The surface energy of a boundary between a normal and superconducting region of the superconductor is proportional to the difference $(\xi-\lambda)$. Consequently the sign of this surface energy depend on the value of GL parameter $k$. When $k<\frac{1}{\sqrt{2}}$, the surface energy is positive and the superconductor is named type I. If $k>\frac{1}{\sqrt{2}}$, the surface energy is negative and the superconductor is classified as type II. In the last type the magnetic field penetrates in small tubes, named vortices, each one carrying a magnetic flux quantum 


$$
\Phi=n \Phi_{0}=n \frac{h c}{2 e}
$$

For type I superconductor exist only one critical field $H_{c}$. Because the phase transition between the superconducting and normal states is of first order, that is, we can observe a discontinuity between both states for a magnetic field $H_{c}$ (see Fig. 2).

$$
H_{c}(T)=\frac{\Phi_{0}}{2 \sqrt{2} \pi \xi(T) \lambda(T)}
$$

For field lower than $H_{c}$ all magnetic field is expelled from the interior of the superconductor and above this the superconductivity is completely destroyed.

The type II superconductor have two critical fields $H_{c 1}$ and $H_{c 2}$. Because the phase transition between both states is of second order,thus, there is no discontinuity at a given $H_{c}$, instead, the superconductor enters a mixed state between $H_{c 1}$ and $H_{c 2}$ (see Fig. 2).

$$
\begin{gathered}
H_{c 1}(T)=\frac{\Phi_{0}}{4 \pi \lambda^{2}(T)} \ln k \\
H_{c 1}(T)=\frac{\Phi_{0}}{2 \pi \xi^{2}(T)} .
\end{gathered}
$$

For field lower than $H_{c 1}$ the superconductor is a perfect diamagnetic, but for field between $H_{c 1}$ and $H_{c 2}$ the superconductivity survive between vortices penetrating the sample

Above the $H_{c 2}$ the superconductivity is destroyed, in bulk samples. For thin samples, superconductivity is still present up to the third critical field $H_{c 3}, H_{c 3}>H_{c 2}$, in filamentary regions of the sample $[53,54]$.

Figure 2 show the magnetization $\mathbf{M}$ as the function of the applied field $\mathbf{H}$. The magnetization is defined by

$$
\mathbf{M}=\frac{\mathbf{B}-\mathbf{H}}{4 \pi}
$$

where $\mathbf{B}$ the magnetic induction. In the Meissner state $B=0$, so $M=-\frac{H}{4 \pi}$. The entrance of vortices cause a decrease in the magnetization until zero. For type $I$ this decrease is abrupt, but for type II superconductor it occurs gradually.

\subsection{Abrikosov vortice}

Alexei Abrikosov [55] explained some important characteristic of type II superconductor. In the region $H_{c 1}<H<H_{c 2}$ the vortex and superconducting states are in equilibrium. In the vortex state, the magnetic field is confined to form magnetic flux lines, the vortices, by the surrounding circulating superconducting current. With increasing applied field, these vortices are arranged in a triangular lattice, known as Abrikosov lattice. 


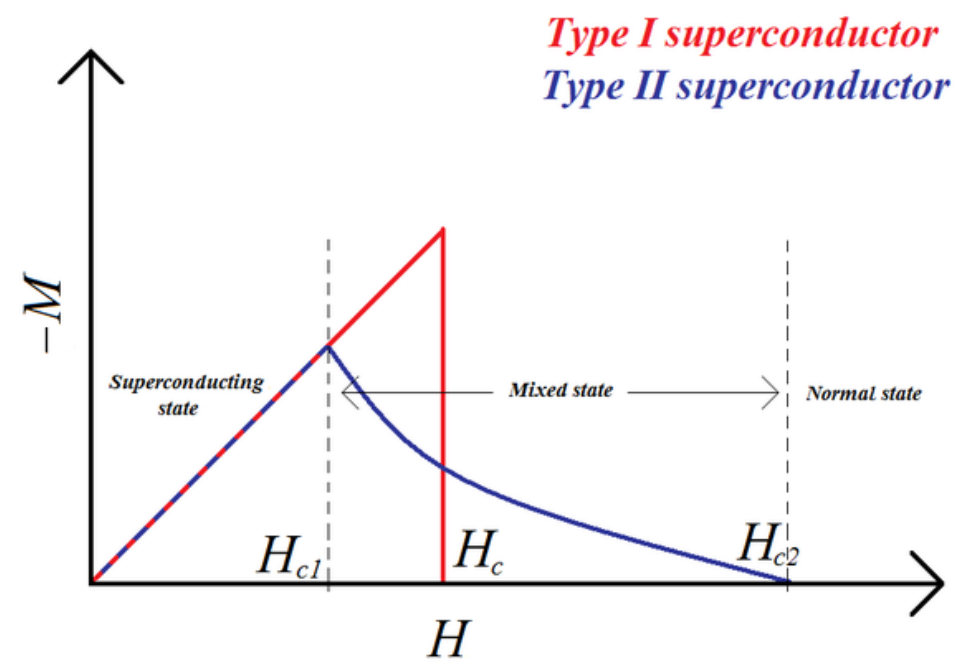

Figure 2: A schematic picture of magnetization curves for bulk type $I$ and type $I I$ superconductors. For type $I$ the phase transition between the superconducting and normal states is of first order. We can observe a discontinuity between both states for a magnetic field $H_{c}$. For type $I I$ superconductor, the phase transition between both states is of second order and there is no discontinuity at a given $H_{c}$, instead, the superconductor enters a mixed state between $H_{c 1}$ and $H_{c 2}$.

\subsubsection{Forces on Abrikosov vortice}

According to $[56,57]$ a vortice of unit length experiment a Lorentz driving force $\mathbf{J} \times \Phi_{0}$ from the current of mean density $\mathbf{J}$ and a pinning force $\mathbf{p}$ from the defects. The critical current density $J_{c}$ is calculated from the following force balance equation just before depinning

$$
\mathbf{J} \times \Phi_{0}+\mathbf{p}_{\max }=0 .
$$

The nature of the driving force has been object of discussion along the years. Some author have changed the sign of the first term of Eq. (2.4.1) [58-61]. The name "Lorentz force" was considered improper because the Magnus force include the Lorentz force and the first one is not a consequence of electromagnetic effects on a vortex [62]. This statement has been widely refuted and the conventional meaning of the Lorentz force is clearly stated and applied [63].

Cheng et al. [64] studied the nature of the driving force on an Abrikosov vortices by using the London equation. It was concluded that the driving force on an Abrikosov vortices is not a magnetic Lorentz force. In the low $\xi / \lambda$ limit, the force is dominated by a kinetic interaction and is proportional to the local densities of vortex and driving current around the vortex core.

Our discussion of following subsection are base in Gennes' classical book [54].

\subsubsection{Energy (E) of Abrikosov vortice}

We assume that a vortice is well pinned so that the more general Magnus force is reduced to the Lorentz force and there is not effect from normal currents. If the supercurrent 
density and magnetic field have a slow space variation, the London equation can be used

$$
\mathbf{H}+\lambda^{2} \nabla \times \nabla \times \mathbf{H}=0
$$

with $\lambda$ the penetration depth.

For extreme type-II superconductor, the magnetic field inside the vortex with hard core of very small radius $\xi$ is given by the expression

$$
\mathbf{H}+\lambda^{2} \nabla \times \nabla \times \mathbf{H}=\Phi_{0} \delta(\mathbf{r}) / \mu_{0} .
$$

The magnetic field is applied along the $z$ axis.

Integrating the Eq. (2.4.3) on a closed path of radius $r$ and using the curl formula, we have

$$
\int \mathbf{H} . d \mathbf{s}+\lambda^{2} \oint \nabla \times \mathbf{H} . d \mathbf{l}=\Phi_{0} / \mu_{0} .
$$

When $r=\xi$ the first integral is negligible compared with the second leading

$$
2 \pi \lambda^{2} r|\nabla \times \mathbf{H}|=\Phi_{0} / \mu_{0}
$$

The solution of Eq. (2.4.3) is

$$
H(r)=\frac{\Phi_{0}}{2 \pi \mu_{0} \lambda^{2}} K_{0}\left(\frac{r}{\lambda}\right)
$$

where $K_{0}$ is the zero-order second-kind modified Bessel function.

Now, the energy of unit length can be calculated as follow

$$
\begin{aligned}
E & =\int\left[\frac{\mu_{0}}{2} \mathbf{H}^{2}+\frac{\mu_{0} \lambda^{2}}{2}(\nabla \times \mathbf{H})^{2}\right] d V \\
& =\frac{\Phi_{0}^{2}}{4 \pi \mu_{0} \lambda^{2}} \ln \left(\frac{\lambda}{\xi}\right) .
\end{aligned}
$$

In this deduction the low $r / \lambda$ limit of Eq. (2.4.6) has been used and the volume integration was taken outside the core. The first and second terms in the integral are field energy and kinetic energy, respectively. The surface integration was selected on cylindrical surface of the core with $d \mathbf{s}$ directed inwards.

\subsubsection{Interaction energy of two Abrikosov vortices}

Now, we consider two vortices in a large superconductor, both parallel to $z$ axis. The equation that determine the field distribution is 


$$
\mathbf{H}+\lambda^{2} \nabla \times \nabla \times \mathbf{H}=\Phi_{0}\left[\delta\left(\mathbf{r}-\mathbf{r}_{1}\right)+\delta\left(\mathbf{r}-\mathbf{r}_{2}\right)\right] / \mu_{0},
$$

with the vortices at the position $\mathbf{r}_{1}=\left(x_{1}, 0\right)$ and $\mathbf{r}_{2}=\left(x_{2}, 0\right)$ respectively.

$\mathbf{H}(r)$ is the superposition of fields of each vortex

$$
\mathbf{H}_{i}(\mathbf{r})=\frac{\Phi_{0}}{2 \pi \mu_{0} \lambda^{2}} K_{0}\left(\frac{\left|\mathbf{r}-\mathbf{r}_{i}\right|}{\lambda}\right)
$$

The interaction energy $E_{12}$ of two vortices can be calculated as

$$
E_{12}=\frac{\mu_{0} \lambda^{2}}{2} \int d \mathbf{s}_{1} \cdot \mathbf{H}_{2} \times \nabla \times \mathbf{H}_{1}+d \mathbf{s}_{2} \cdot \mathbf{H}_{1} \times \nabla \times \mathbf{H}_{2}
$$

The integration was performed over cylindrical surface of the cores of both vortices. In the same way of Eq. (2.4.5) it is obtained

$$
2 \pi \lambda^{2}\left|\mathbf{r}-\mathbf{r}_{i}\right|\left|\nabla \times \mathbf{H}_{i}\right|=\Phi_{0} / \mu_{0}\left(\left|\mathbf{r}-\mathbf{r}_{i}\right|=\xi\right)
$$

and

$$
E_{12}=\Phi_{0} H_{12}
$$

,where

$$
H_{12}=H_{1}\left(\mathbf{r}_{2}\right)=H_{2}\left(\mathbf{r}_{1}\right)=\frac{\Phi_{0}}{2 \pi \mu_{0}^{2}} K_{0}\left(\frac{x_{2}-x_{1}}{\lambda}\right) .
$$

$E_{12}$ decrease with increasing $x_{2}-x_{1}$, then it is a repulsive energy. The Ampere law is employed for calculating the force on the second vortex obtaining

$$
F_{2 x}=\Phi_{0} J_{1 y}\left(\mathbf{r}_{2}\right)
$$

and $J_{1 y}$ is the density of the current of the first vortex at $\mathbf{r}_{2}$, where the small cylindrical core of the second vortex is located. The last equation can be written as

$$
\mathbf{F}_{2}=\mathbf{J}_{1}\left(\mathbf{r}_{2}\right) \times \Phi_{0}
$$

and this is agrees with definition of driving force in Eq. (2.4.1).

\subsubsection{Quantization of magnetic flux and kinetic nature of driving force}

We will present the kinetic nature of driving force as reported in [64].

It is know that $\Phi_{0}$ is a magnetic flux quantum $\left(\Phi_{0}=\pi \hbar / e=2.07 \times 10^{-15} \mathrm{~Wb}\right)$, but we will see this more detailed. The vector potential $\mathbf{A}$ and the current density $\mathbf{J}$ can be included in Eq. (2.4.3) as follow 


$$
\nabla \times \mathbf{A}+\mu_{0} \lambda^{2} \nabla \times \mathbf{J}=\Phi_{0} \delta(\mathbf{r})
$$

leading to

$$
\mathbf{J}=\frac{\hbar e n}{2 m}\left(\nabla \theta-\frac{2 e}{\hbar} \mathbf{A}\right)
$$

where we have used the definition of $\lambda$ (Eq. 2.2.12) and remembered that the supercurrent is defined by $\mathbf{J}=-n e \mathbf{v}$. Here $\theta$ is the phase of superconducting order parameter. The order parameter must be single valued, so making an integration over the interior surface of a circle, as done for Eq. (2.4.4) and obtaining the vorticity of the phase $\theta$,

$$
\frac{1}{2 \pi} \oint \nabla \theta .1=1
$$

valid only if $\Phi_{0}=\pi \hbar / e=2.07 \times 10^{-15} \mathrm{~Wb}$.

$\Phi_{0}$ can be understood in two ways:

- The first as the total flux carried by a complete Abrikosov vortex. For $r \gg \lambda$, the line integral in Eq. (2.4.4) can be ignored due to the high-degree small boundary current. So $\mu_{0} \int \mathbf{H} . d \mathbf{s}=\Phi_{0}$ and $\Phi_{0}$ is referred to as the flux quantum and the driving force in Eq. (2.4.15) seems to be electromagnetic.

- On the other hand if the radius of circle of integration is very small, Eq. (2.4.4) leads to Eq. (2.4.5). So $\Phi_{0}$ is also the circulation of the current density around the circle of integration multiplied by $\mu_{0} \lambda^{2}$. Then the driving force in Eq.(2.4.15) is exerted between two currents. For illustrating this point, we can use Eqs. (2.4.10) and (2.4.11) and the Ampere law to rewrite Eq. (2.4.14) as

$$
\begin{aligned}
F_{2 x} & =-\mu_{0} \lambda^{2} 2 \pi \xi\left|\nabla \times \mathbf{H}_{2}\right| \partial H_{1} / \partial x_{2} \\
& =\mu_{0} \lambda^{2} 2 \pi \xi J_{2}\left(\left|\mathbf{r}-\mathbf{r}_{2}\right|=\xi\right) J_{1}\left(\left|\mathbf{r}-\mathbf{r}_{2}\right|=\xi\right)
\end{aligned}
$$

This equation shows that the driving force $F_{2 x}$ on the second vortex does not explicity depend on the total flux $\Phi_{0}$ it carries. It is proportional to the circulation of its own current density along the border of the core, $2 \pi \xi J_{2}\left(\left|\mathbf{r}-\mathbf{r}_{2}\right|=\xi\right)$, and the current density of the first vortex on the same border, $J_{1}\left(\left|\mathbf{r}-\mathbf{r}_{2}\right|=\xi\right)$. As $J=-n e \mathbf{v}$, with $\mathbf{v}$ the electron velocity, the force between two currents should have a kinetic origin.

From Eq. (2.4.6) we can calculated the field energy of vortex obtaining

$$
E_{h}=\frac{\Phi_{0}^{2}}{4 \pi \mu_{0} \lambda^{2}} \int_{\xi / \lambda}^{\infty} x K_{0}^{2}(x) d x=\frac{\Phi_{0}^{2}}{8 \pi \mu_{0} \lambda^{2}},
$$


when $\xi / \lambda \ll 1$. $E_{h}$ is negligible small in the low $\xi / \lambda$ limit when this is compared with the total energy expressed by Eq. (2.4.7). We can conclude that the total energy is dominated by the kinetic energy. The situation is similar for the energy $E_{12}$. This leads the conclusion that the nature of the driving force should also be mainly kinetic and the relevant meaning of the $\Phi_{0}$ appearing in Eq. (2.4.15) is kinetic too. In the Anderson and Kim's work [57] the interaction energy was treated as an obvious consequence of magnetic energy and considered the driving force as Lorentz force.

Many works evidence the kinetic nature of driving force $[65,66]$.

It is not obvious that the Lorentz force arises in a framework theory where the magnetic effects of the circulating currents is neglected. But, effectively, when the vortex core does not carry any flux, the nature of $\Phi_{0}$ in Eq. (2.4.1) is not magnetic but kinetic, and the driving force can be calculated using a formula containing $\Phi_{0}$ [64]. Remembering that, $\mathbf{J}_{1}=-n e \mathbf{v}$, and $\Phi_{0}=\pi \hbar / e$, Eq. (2.4.15) becomes

$$
\mathbf{F}=-\pi \hbar n \mathbf{v} \times \mathbf{k}
$$

with $\mathbf{k}$ the unit vector along the $z$ axis.

The last relation has no explicit relation to $e$ and $m$. But is proportional to the electron velocity and the electron density. Except for a sign difference, this is consistent with Eq. (9) of [62] for the Magnus force, which was derived in terms of the geometric phase. So, we can generally say that the driving force is a dynamical quantum-mechanical force on the vortice. This force can be expressed electromagnetically as Eq. (2.4.15), however this hide the actual nature of the driving force and give rise to a sign confusion. The driving force can also be expressed kinetically as

$$
\mathbf{F}=-n m \mathbf{v} \times \Omega_{0}
$$

where $\Omega_{0}$ is the vectorial quantum of the electron-velocity circulation closely around the vortex core,

$$
\Omega_{0}=\pi \hbar / m=\Phi_{0} e / m=3.637 \times 10^{-4} \mathrm{~m}^{2} / \mathrm{s} .
$$

The kinetic nature of the driving force is explicit in Eq. (2.4.22) and Eq. (2.4.23) explains precisely the kinetic meaning of $\Phi_{0}$.

It is necessary to emphasize that the nature of driving force depend of the value of $\xi / \lambda$. In the low $\xi / \lambda$ limit the nature of driving force is kinetic, but as $\xi / \lambda$ cannot be zero in any real case and $\xi / \lambda>0$, the energy will have both the kinetic and magnetic contributions. In both case the driving force is described by the same function. 


\subsection{Flux pinning}

When an external current is applied to superconductor, there is a critical current at which the vortices start to move transverse to the applied current density $j$. The Lorentz force per unit volume is given by

$$
\mathbf{F}_{L}=\mathbf{j} \times \frac{\mathbf{B}}{c} .
$$

The electrical field induced by the moving vortices is

$$
\mathbf{E}=\mathbf{B} \times \frac{\mathbf{v}}{c}
$$

Where $\mathbf{v}$ is the velocity of vortex lattice. This electric field produces an energy dissipation in the superconductor. The pinning force $\mathbf{F}_{p}$ is introduce in order to compensate the Lorentz force and consequently the superconductor can support higher currents with lower dissipation energy. The total force that vortex experiment is

$$
\mathbf{F}=\mathbf{F}_{L}-\mathbf{F}_{p}-\mathbf{F}_{M}-\eta \mathbf{v}
$$

where $-\eta \mathbf{v}$ and $\mathbf{F}_{M}$ are a viscous damping and Magnus forces respectively.

There are several pinning mechanisms that can be divided in two principal groups [67]: The electromagnetic pinning and the core pinning. In the first group the responsible for pinning are the supercurrents around the vortices and the local magnetic fields produced by the defects. The kinetic energy of the supercurrents can be lowered when vortices are situated on the pinning sites, resulting in an attraction between vortices and pinning centers. The important length scale here is the penetration depth $\lambda$. In the second group the attractive interaction between vortices and defects is consequence of the minimization of free energy when the vortex core is located at the position where $T_{c}$ or $\kappa$ have a local variation. More effective core pinning is achieved when the size of the pinning site is of order $\xi$ or $\lambda$.

The effect of pinning is to strengthen the superconductivity. The pinning defects pin the vortices causing a decrease of energy dissipation when a external current is applied. Artificial pinning centres, such as antidots [68-70] or magnetic dots [71-73], are fabricated on nano-scale. When the stable vortex configurations reproduce the symmetry of the pinning array, a local enhancement of the magnetization $\mathrm{M}(\mathrm{H})$ and the critical current is achieved. These pronounced maxima are named matching features and occur at integer multiples of the first matching field, which is defined as the field at which the density of vortices equals the density of pinning sites. The first observation of the matching phenomena in one dimension was reported in [74] . Further experiments with two-dimensional arrays of holes were reported $[75,76]$. Later superconductors with a square and triangular arrays of pinning centers were studied [70,77]. The maximum number of flux quanta that 
can be trapped inside a pinning center, named saturation number, depends on the ratio between the size of the pinning site and the coherence length [78-80].

Recently, Machida [1] studied the effect of a triangular array of pinning centers in the properties of vortex lattice. The calculations were performed in a two-dimensional square with current $j$ flowing, as schematically shown in Fig 3. The pinning centers are square of size $1.4 \xi_{0} \times 1.4 \xi_{0}$. The dynamics of the domain wall and half flux-quantum vortices of the vortex sheet structure were investigated. The domain wall moves with the flux flow both in the free flux flow case and the pinning case. It was observed the creation process in a way that a pair of half flux-quantum vortices is changed to a conventional vortex and liberated from the vortex sheet.

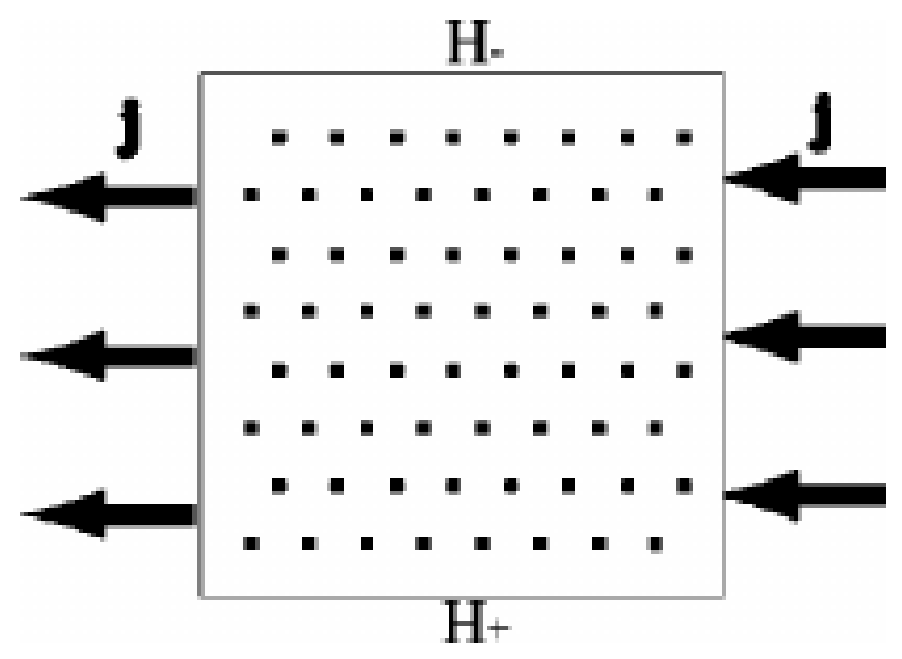

Figure 3: Configuration of the simulation. The current density $\mathbf{j}$ flows in at righ-hand side boundary and out at the left-hand side boundary of square superconductor. External fields $H_{+}$and $H_{-}$are applied outside the lower and upper boundaries, respectively. Pinning centers, where superconductivity is suppressed are represented by small squares. Taken from Ref. [1].

Jelic et al., [2] investigated the fundamental consequences of a temporally periodic pinning landscape imprinted on a superconducting condensate. It was considered a superconducting stripe with longitudinally applied current, in magnetic field perpendicular to its plane, and with an oscillating depletion line along its middle (See Fig. 4). The generalized timedependent Ginzburg-Landau (TDGL) equations were used to calculated the voltage as function of the applied current, magnetic field, material parameters and the period of the time dependent pinning. In the TDGL was included the function $f(t, \mathbf{r})$ for the pinning as follow

$$
\frac{u}{\sqrt{1+\gamma^{2}|\Psi|^{2}}}\left(\frac{\partial}{\partial t}+\frac{\gamma^{2}}{2} \frac{\partial|\Psi|^{2}}{\partial t}\right)|\Psi|=(\nabla-i \mathbf{A})^{2}|\Psi|+\left(f(t, \mathbf{r})-|\Psi|^{2}\right) \Psi
$$




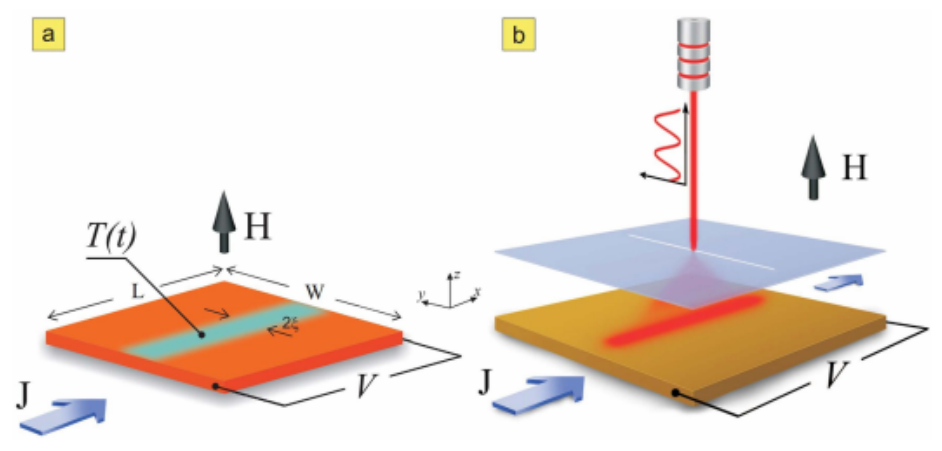

Figure 4: (a) A superconducting stripe of width $W$ with a central, time-dependent, pinning line of width comparable to the vortex size, and a 4-point probe applied $d c$ current (with density $J$ ), and measured voltage $(V$ at contacts separated by distance $L)$. A depletion region is simulated as sinusoidally oscillating local heating up to the critical temperature $T_{c}$ and back to working temperature, with frequency $\omega$. (b) Possible experimental setup corresponding to (a), by using laser light at the far field region passing through a metallic mask to create time-dependent and spatially modulated depletion of the superconducting condensate. Taken from Ref. [2].

$$
\frac{\partial \mathbf{A}}{\partial t}=\operatorname{Re}\left\{\Psi_{*}(-i \nabla-\mathbf{A}) \Psi\right\}-\kappa^{2} \nabla \times \nabla \times \mathbf{A}
$$

These equations are expressed in the dimensionless units, where the order parameter, $\Psi$, is given in the units of $\Delta(T)=4 k_{B} T_{c} u^{1 / 2} / \pi \sqrt{1-T / T_{c}}\left(k_{B}\right.$ is the Boltzmann constant, $u=5.79$ is the ratio of the relaxation time of the order parameter phase and the relaxation time of the order parameter amplitude), $\mathbf{A}$ is in units of $\Phi_{0} / 2 \pi \xi(T)$. The parameter $\gamma=2 \tau_{i n} \Delta(T) / \hbar$. Time is expressed in the units of Ginzburg-Landau relaxation time, $\tau_{G L}=\pi \hbar / 8 k_{B}\left(T_{c}-T\right) u$, and all of the distances are scaled with the coherence length, $\xi(T)$.

The time-dependent pinning potential is given by:

$$
f(t, \mathbf{r})= \begin{cases}1, & \text { out of the depletion region } \\ \frac{1}{2}\left(1-\cos \frac{2 \pi t}{\tau}\right), & \text { in depletion region }\end{cases}
$$

The term $2 \pi / \tau$ is the angular frequency $\omega$ of the pinning. The function $f(t, \mathbf{r})$ provides oscillations of the order parameter in the depletion region, which shifts between the superconducting state $(f=1)$, and normal state $(f=0)$.

It was observed a temporally matching phenomena caused by stroboscopic commensurability between the characteristic frequency of the vortex motion under applied current and the frequency of the dynamic pinning. This leads to unusual features such as externally variable resistance/impedance and Shapiro steps in current-voltage characteristic. 


\subsection{Kosterlitz-Thouless transition}

The focus of this thesis is the study of vortex dissociation process. We consider important to describe the existing theories about the decoupling of composite vortex. There is the reason why we expose the Kosterlitz-Thouless transition here.

In several physical system components at one level go together and form certain collective coherent structures, such as topological defects or topological charges. Among them we have Coulomb charges in two dimensions, dislocations in two dimensional crystals, and of our particular interest, vortices in two dimensional superconductors. The interaction between the topological charges depends in all cases logarithmically on the spatial separation and this leads to some very general collective behaviour, most spectacularly it causes a certain type of phase transition namely Kosterlitz-Thouless transition [81].

The Berezinskii-Kosterlitz-Thouless transition (BKT transition) is a phase transition in the two-dimensional (2-D) XY model. It is a transition from bound vortex-antivortex pairs at low temperatures to unpaired vortices and anti-vortices at some critical temperature. The transition is named for condensed matter physicists Vadim Berezinskii, John M. Kosterlitz and David J. Thouless. BKT transitions can be found in several 2-D systems in condensed matter physics that are approximated by the XY model, including Josephson junction arrays and thin disordered superconducting granular films.

\subsubsection{The two dimensional XY-model}

The hamiltonian of planar rotor of unit length arranged on a two dimensional square lattice is

$$
H=-J \sum_{<i j>} \mathbf{S}_{i} \cdot \mathbf{S}_{j}=-J \sum_{<i j>} \cos \left(\theta_{i}-\theta_{j}\right)
$$

$<i j>$ denotes summation over all nearest neighbour sites in the lattice, and $\theta_{i}$ denotes the angle of the rotor on site $i$ with respect to some polar direction in the two dimensional vector space containing the rotors.

The cos function can be aproximated by Taylor expansion assuming that the direction of the rotor varies smoothly from site to site. The sum over the nearest neighbours corresponds to the discrete Laplace operator, which can be expressed as

$$
\theta_{i}-\theta_{j}=\partial_{x} \theta
$$

for two site $i$ and $j$ which differs by one lattice spacing in the $x$-direction. The hamiltonian take the integral form

$$
H=E_{0}+\frac{J}{2} \int d \mathbf{r}(\nabla \theta)^{2} .
$$


$E_{0}=2 J N$ is the energy of the completely aligned ground state of $N$ rotors.

The partition function of the system is obtained by

$$
Z=e^{-\beta E_{0}} \int D[\theta] \exp \left\{-\beta \frac{J}{2} \int d \mathbf{r}(\nabla \theta)^{2}\right\}
$$

The integral over $\theta(\mathbf{r})$ can be divided into a sum over the local minima $\theta_{v}$ of $H[\theta]$ plus fluctuation $\theta_{s \omega}$ around the minima

$$
\begin{aligned}
Z=e^{-\beta E_{0}} \sum_{\theta_{v}} \int D\left[\theta_{s \omega}\right] & \\
& \exp \left\{-\beta\left(H\left[\theta_{v}\right]+\frac{1}{2} \int d \mathbf{r}_{1} \int d \mathbf{r}_{2} \theta_{s \omega}\left(\mathbf{r}_{1}\right) \frac{\delta^{2} H}{\delta \theta\left(\mathbf{r}_{1}\right) \delta \theta\left(\mathbf{r}_{2}\right)} \theta_{s \omega}\left(\mathbf{r}_{2}\right)\right)\right\}
\end{aligned}
$$

The field configuration corresponding to local minima of " $\mathrm{H}$ " are solutions to the external condition

$$
\frac{\delta H}{\delta \theta(\mathbf{r})}=0 \Rightarrow \nabla^{2} \theta(\mathbf{r})=0 .
$$

Trivial solution to this equation correspond to the ground state $\theta(\mathbf{r})=$ constant .

The other solution is obtained by imposing the following boundary conditions on the circulation integral of $\theta(\mathbf{r})$ :

$$
\oint \nabla \theta(\mathbf{r}) \cdot d \mathbf{l}=2 \pi n
$$

Here $n \neq 0$ for all closed curves encircling the position $\mathbf{r}_{0}$ of the centre of the vortex and $n=0$ for all paths that don't encircle the vortex position $\mathbf{r}_{0}$.

The condition $n \neq 0$ imposes a singularity in the director field. The circulation integral must be equal to an integer times $2 \pi$ since we circle a closed path and therefore $\theta(\mathbf{r})$ has to point in the same direction after traversing the path as it did when we started.

The energy of vortex can be estimated considering a spherical symmetric, hence the vortex field $\theta_{v}$ must be of the form $\theta(\mathbf{r})=\theta(r)$. The dependence on $r$ is found from Eq. (2.6.7). The circulation integral along a circle of radius $r$ centred at the position $\mathbf{r}_{0}$ of the vortex is

$$
2 \pi n=\oint \nabla \theta(\mathbf{r}) \cdot d \mathbf{r}=2 \pi r|\nabla \theta|
$$

This implies that

$$
|\nabla \theta|=n / r .
$$

Replacing this result in Eq. (2.6.3) 


$$
E_{v}-E_{0}=\frac{J}{2} \int d \mathbf{r}[\nabla \theta(\mathbf{r})]^{2}=\pi n^{2} J \ln \left(\frac{L}{a}\right) .
$$

$\nabla \theta$ decays only as $1 / r$ leading to a logarithmic divergence of the energy. Consequently the integral over $r$ in Eq. (2.6.10) is cut-off for large $r$-values by the finite system size $L$ and for small $r$-values by the lattice spacing $a$. The factor $n$ in Eq. (2.6.7) is called winding number. The energy of the vortex is quadratic in the $n$, then the vortex with $n>1$ are not favorable. This type of vortex is named giant-vortex.

Consider a pair of single charged vortex and anti-vortex. For the vortex the integral in Eq. (2.6.7) is equal to $2 \pi$ and for anti-vortex is equal $-2 \pi$. Hence if we select a path enclosing both vortices the result of integral is zero $(0)$. The distortion of the phase field $\theta(\mathbf{r})$ from the vortex-anti-vortex pair is able to cancel out at distances from the center of the two vortices large compared to the separation $R$ between the vortex and the anti-vortex. This explains why the energy of the vortex pair is of the form

$$
E_{2 v}(R)=2 E_{c}+E_{1} \ln \left(\frac{R}{a}\right),
$$

with $E_{c}$ the energy of the vortex cores and $E_{1}$ is proportional to $J$. The phase field $\theta_{2 v}(\mathbf{r})$ of a vortex $\mathbf{r}=(-a, 0)$ and anti-vortex located a $\mathbf{r}=(a, 0)$ is

$$
\theta_{2 v}(R)=\operatorname{arctg}\left(\frac{2 a y}{a^{2}-r^{2}}\right)
$$

\subsubsection{Vortex unbinding}

The free energy of a single vortex is calculated by $F=E-T S$. The energy $E$ is given by Eq. (2.6.10) and the entroly $S$ is $S=k_{B} \ln \left(L^{2} / a^{2}\right)$. Then

$$
F=E_{0}+\left(\pi J-2 k_{B}\right) \ln (L / a)
$$

For $T<\pi J / 2 k_{B}$ the free energy diverge to plus infinity as $L \rightarrow \infty$. For $T>\pi J / 2 k_{B}$ the system can lower its free energy by producing vortices, hence $F \rightarrow-\infty$ as $L \rightarrow \infty$. This simple heuristic argument points to the fact that the logarithmic dependence on system size of the energy of the vortex combines with the logarithmic dependence of the entropy to produce the subtleties of the vortex unbinding transition. It is the logarithmic size dependence of the $2 \mathrm{~d}$ vortex nergy that allows the outcome of the competition between the entropy and the energy to change qualitatively at a certain finite temperature $T_{K T}$.

\subsubsection{The spin wave stiffness}

The spin wave stiffness $\rho_{s}^{R}$ depends on the temperature and is defined to describe the effect of the thermally activated vortex pairs. This is the case of what is named a generalised 
rigidity [82]. $\rho_{s}^{R}$ gives the energy necessary to apply a twist, or gradient, to the rotors or spins:

$$
\theta(\mathbf{r})=\theta_{0}(\mathbf{r})+\mathbf{v}_{e x} \cdot \mathbf{r},
$$

where $\theta_{0}(\mathbf{r})$ vary according to the canonical ensemble and $\mathbf{v}_{e x}$ is the applied gradient. The free energy is increased by

$$
F\left(\mathbf{v}_{e x}\right)-F(0)=\frac{1}{2} V \rho_{s}^{R} v_{e x}^{2} .
$$

In the superconductivity phenomena $\theta(\mathbf{r})$ is the phase of the complex order parameter. The superscript $R$ in $\rho_{s}^{R}$ indicates that thermal excitations renormalise the quantity. From the hamiltonian (Eq. 2.6.3) follows immediately that at zero temperature $\rho_{s}^{R}=J=\rho_{s}$. The spin wave stiffness is similar to the shear constant of material. The shear constant says the energy cost of a shear deformation. As temperature is increased the shear constant decreases and drops abruptly to zero when the solid melts into a liquid.

The detail for calculating $\rho_{s}^{R}$ is found in [83]. To obtain $\rho_{s}^{R}$ one calculates the left hand side of Eq. (2.6.15). The phase field is split into two parts

$$
\theta_{0}(\mathbf{r})=\theta_{s}(\mathbf{r})+\theta_{v}(\mathbf{r})
$$

The first term describes smooth spin waves and the second term contain the singular vortex contribution. From the thermodynamic theory $F=K_{b} T \ln Z$ and the Eq. (2.6.4) it is obtained the free energy

$$
\rho_{s}^{R}=\rho_{s}-\frac{1}{2} \frac{\rho_{s}^{2}}{T} \lim _{k \rightarrow 0} \frac{\langle\widehat{n}(\mathbf{k}) \widehat{n}(-\mathbf{k})\rangle}{k^{2}},
$$

where the Fourier transforms of the phase field has been used. $n(\mathbf{r})$ is the correlation function of the Fourier transform of the vortex density function

$$
n(\mathbf{r})=\sum_{\alpha} n_{\alpha} \delta\left(\mathbf{r}-\mathbf{r}_{\alpha}\right)
$$

for a collection of vortices of density $n_{\alpha}$ with centres located at positions $\mathbf{r}_{\alpha}$.

\subsubsection{The KT transition}

The number of vortex pairs thermally activated increases with increasing temperature. In consequence $\rho_{s}^{R}$ decrease. This correspond to a decrease in the increment of the free energy induced by a certain twist $\mathbf{v}_{e x}$. This is explained because the phase field $\theta(\mathbf{r})$ becomes more and more distorted as the temperature is increased, hence the extra perturbation caused by $\mathbf{v}_{e x}$ becomes relatively less important. The spin wave stiffness is given by 


$$
\rho_{s}^{R}\left(T_{K T}^{-}\right)= \begin{cases}\rho_{s}^{R}\left(T_{K T}^{-}\right)\left[1+\text { const. }\left(T_{K T}-T\right)^{1 / 2}\right], & \text { for } T<T_{K T} \\ 0, & \text { for } T>T_{K T}\end{cases}
$$

where $T_{K T}$ is the Kosterlitz-Thouless temperature at which vortex pairs unbind. In the 2d $X Y$-model $T_{K T} / J \simeq 0.893 \pm 0.002$ [84].

All systems that undergoes a $K T$-transition obey the universal relation

$$
\rho_{s}^{R}\left(T_{K T}^{-}\right) / T_{K T}=\frac{2}{\pi},
$$

Since $\rho_{s}^{R}\left(T_{K T}^{-}\right)=0, \mathrm{Eq}(2.6 .20)$ is referred as the universal jump. The correlation length $\xi(T)$ has an unusual behavior as one approaches $T_{K T}$ from above. There is a slow divergence of the $\xi(T)$ as the critical temperature is approached. However, the $K T$-transition diverge much faster, then

$$
\xi(T) \sim \exp \left(\frac{\text { const. }}{\left(T-T_{K T}\right)^{1 / 2}}\right) \text { for } T>T_{K T} .
$$

\subsection{Multicomponent superconductivity based on multiband superconductors}

It is considered that multiband superconductors present multicomponent superconductivity when an interband pairing interaction is considerably weaker than the intraband interactions. In this way each band has a condensate with an amplitude and phase that weakly interacts with the condensates of the other bands. The system has multiple quantum phases, which is not a straightforward extension of a conventional single-band superconductor with only one quantum phase.

To date many materials are useful for studying the multicomponent superconductivity based on multiband superconductors. The transition metals, Nb and V, have been considered to present a multiband nature [85,86]. Similarly $\mathrm{NbSe}_{2}$ [87], graphite intercalation compounds [88,89] and Chevrel compound [90] were investigated. Multilayer cuprate superconductors exhibits the multiband nature [91-94]. $\mathrm{MgB}_{2}[6]$ and pnictides [95] are also considered as attractive candidates.

The original BCS theory of superconductivity was a simplified model for an isotropic single superconducting gap [3]. Suhl, Matthias and Walker proposed two-band extension in 1959 [5]. Two different magnitudes are assigned to two different superconducting gaps, whereas the superconducting phase is identical for the two gaps. In 1962, Kondo [77] and Peretti [96] independently introduced different phases for different gaps due to the nonelectron phonon-mediated superconductivity speculated to exist owing to the vanishing of the isotope effect in some transition metal superconductor, such as Ru and Os. The phase difference between two gaps is $\pi$ radians, indicating sign reversal gaps. Leggett introduced a phase difference other than 0 or $\pi$ radians as a collective excitation potentially 
present in the transition metal superconductor [10]. Tanaka extended this fluctuation to $2 \pi$ radians [11], leading an interband phase-difference soliton ( $i$ soliton). In multilayer cuprate superconductor $\mathrm{Cu}-1234$ was considered the topology and multiple components with regard to the superconductivity in the multiband superconductor. To introduce the multicomponent picture for multiband superconductor allow to examine the physics involving a field theory admitting multiple gauge field [79, 97-103]. The physics of $\mathrm{MgB}_{2}$ and pnictides has been frequently discussed according to this picture since 2001 [80].

The terms multiband superconductors and multigap superconductors do not always indicate the same meaning and physics, which seem to vary by researchers and subjects.

It is important to remark that all real superconductors have the ability to change the isotropic magnitude, phase, and temperature evolution of the gap. These modifications can be introduced by the effects of the anisotropy of the Fermi surface [104] and pairing interaction [105], which modify the magnitude of the gap. Gross, Massidda, and their collaborators are constructing an ultimate modern version of the $a b$ initio calculation of the anisotropic gap [106].

Other way to modify the isotropic gap is introducing two discrete magnitudes into the gaps, as Suhl, Mattias, and Walker did [5]. Two gaps originate from two bands. We have three cases to consider separately: In the first case the magnitude of the gap on one band is different from that on another band. For the second case, the superconductivity can emerge by the interband interaction only without the intraband interaction. In this case, the temperature dependence of the magnitude of the gap normalized by that at $0 \mathrm{~K}$ for each band is similar to that of the isotropic single-gap case. It is, the behaviour is similar to the single component superconductivity. Thirdly, the temperature dependence of the gap normalized by that at $0 \mathrm{~K}$ differs considerably from that of the isotropic single gap case when the interband interaction is far smaller than the intraband interaction. That is, it behaves as a double-component superconductivity.

In [5] the condition of the multiband is converted into the pair interaction and density of the state. Within the same band, the magnitude of the intraband pair interaction is the same, as is the density of the state. There is a difference in the magnitude of the intraband pair interaction and the density of the state between two bands. The magnitude of the interband interaction is also the same throughout the Fermi surface. Suhl et. al, discussed two cases: One exhibited a contrast in the strength between two intraband interaction or in the density of the state between two bands, causing two gaps, and the other exhibited a contrast in the strength between the intraband interaction and interband interaction, causing two components when the interband interaction was very weak. Each band could have any phase and magnitude of the gap. This is the multicomponent superconductivity based on multiband superconductors. 


\subsection{Two-component London superconductivity model}

In the London limit the amplitude of order parameter, $|\Psi(\mathbf{r})|$, in the Ginzburg-Landau free energy (Eq. 2.2.4) is considered constant. The uniform solution of GL equation (2.2.6) is obtained by setting the vector potential to zero, as well as the derivatives of the order parameter. The solution obtained is $\left|\Psi_{0}(\mathbf{r})\right|^{2}=-\frac{\alpha}{\beta}$. For the case of two bands the order parameter can be write as

$$
\Psi_{j}(\mathbf{r})=\left|\Psi_{0 j}(\mathbf{r})\right| e^{i \theta(j)},
$$

with $j=1,2$ the band index. Replacing Eq. (2.8.1) in Eq. (2.2.4), the London free energy density is obtained

$$
F_{L}=\sum_{j=1,2} \frac{1}{2}\left|\Psi_{j}\right|^{2}\left(\nabla \theta^{(i)}+\mathbf{A}\right)^{2}+\frac{1}{2 e^{2}}(\nabla \times \mathbf{A})^{2}+\gamma\left|\Psi_{1}\right|\left|\Psi_{2}\right| \cos \left(\theta^{(1)}-\theta^{(2)}\right),
$$

where the last term is the Josephson interaction.

The last equation can be write as

$$
\begin{aligned}
F_{L}=\frac{1}{2} \sum_{j=1,2}\left|\Psi_{j}\right|^{2}\left(\nabla \theta^{(i)}+\mathbf{A}\right)^{2}+\frac{1}{2} \lambda^{2}\left(\left|\Psi_{1}\right|^{2}\right. & \left.+\left|\Psi_{2}\right|^{2}\right)(\nabla \times \mathbf{A})^{2} \\
& +\frac{1}{\xi_{J}^{2}} \frac{\left|\Psi_{1}\right|^{2}\left|\Psi_{2}\right|^{2}}{\left|\Psi_{1}\right|^{2}+\left|\Psi_{2}\right|^{2}} \cos \left(\theta^{(1)}-\theta^{(2)}\right),
\end{aligned}
$$

where $\lambda$ is the penetration depth and $\xi_{J}$ the Josephson length:

$$
\begin{gathered}
\lambda=\frac{1}{e \sqrt{\left|\Psi_{1}\right|^{2}+\left|\Psi_{2}\right|^{2}}}, \\
\xi_{J}=\sqrt{\frac{\left|\Psi_{1}\right|\left|\Psi_{2}\right|}{\gamma\left(\left|\Psi_{1}\right|^{2}+\left|\Psi_{2}\right|^{2}\right)}} .
\end{gathered}
$$

2.9 Two-component Ginzburg-Landau (TCGL) model

The Gibbs energy functional of the TCGL model is defined by its energy density

$$
\begin{aligned}
g= & \frac{\left(\mathbf{B}-\mathbf{B}_{0}\right)^{2}}{8 \pi}+\sum_{j=1,2}\left\{\frac{1}{2 m_{j}}\left|\mathbf{D} \Psi_{j}\right|^{2}+\alpha_{j}\left|\Psi_{j}\right|^{2}\right. \\
& \left.+\frac{1}{2} \beta_{j}\left|\Psi_{j}\right|^{4}\right\}+g_{J}, \quad g_{J}=-\Gamma\left(\Psi_{1}^{*} \Psi_{2}+\Psi_{1} \Psi_{2}^{*}\right),
\end{aligned}
$$

where $\Psi_{1}=\left|\Psi_{1}\right| \exp \left(i \theta_{1}\right)$ and $\Psi_{1}=\left|\Psi_{1}\right| \exp \left(i \theta_{2}\right)$ are complex gap (condensate) functions of the bands $j=1,2, \mathbf{D}=-i \hbar \nabla-2 e \mathbf{A} / \mathbf{c}$ is the gauge-invariant gradient, $\mathbf{B}=[\nabla \times \mathbf{A}]$ 
is the magnetic field, $\mathbf{B}_{0}$ is the applied (external) field, $g_{J}$ is the interband coupling or the Josephson energy, $\alpha_{j}, \beta_{j}, m_{j}$ are material constants defined as

$$
\begin{aligned}
& \alpha_{j}=-N(0) n_{j} \chi_{j}, \quad \beta_{j}=N(0) \frac{n_{j}}{W^{2}}, \\
& m_{j}=\frac{3 W^{2}}{N(0) n_{j} v_{j}^{2}}, \quad \Gamma=N(0) \frac{\lambda_{12}}{G}, \\
& W^{2}=\frac{8 \pi^{2} T_{c}^{2}}{7 \zeta(3)}, \quad \chi_{j}=\tau-\frac{S_{j}}{n_{j} G},
\end{aligned}
$$

where $\tau=1-T / T_{c}, N_{j}=N(0) n_{j}$ is the band density of states (DOS) at the Fermi energy, $N(0)=N_{1}+N_{2}$ is the total DOS $\left(n_{1}+n_{2}=1\right), \lambda_{i j}=g_{i j} N(0)$ are dimensionless coupling constants for the coupling constants $g_{i j}, G=\lambda_{11} \lambda_{22}-\lambda_{12}^{2}, v_{j}$ is the band Fermi velocity and $S_{1}, S_{2}$ appears in the solution of the linearised gap equation for the critical temperature $T_{c}$ and are defined similar to the earlier works [107]

$$
\begin{aligned}
& S_{1}=\lambda_{22}-n_{1} G S, \quad S_{1}=\lambda_{11}-n_{2} G S, \\
& S=\frac{n_{1} \lambda_{11}+n_{2} \lambda_{22} \pm \sqrt{\left(n_{1} \lambda_{11}+n_{2} \lambda_{22}\right)^{2}+4 n_{1} n_{2} \lambda_{12}^{2}}}{2 n_{1} n_{2} G} .
\end{aligned}
$$

The time-dependent TCGL equations are found from the stationary condition of the energy functional with the additional dynamical contributions, this yields

$$
\begin{aligned}
& \eta_{1} \frac{\partial \Psi_{1}}{\partial t}=\frac{1}{2 m_{1}} \mathbf{D}^{2} \Psi_{1}+\alpha_{1} \Psi_{1}+\left|\Psi_{1}\right|^{2} \Psi_{1}-\Gamma \Psi_{2} \\
& \eta_{2} \frac{\partial \Psi_{2}}{\partial t}=\frac{1}{2 m_{2}} \mathbf{D}^{2} \Psi_{2}+\alpha_{2} \Psi_{2}+\left|\Psi_{2}\right|^{2} \Psi_{2}-\Gamma \Psi_{1}
\end{aligned}
$$

where we introduce the relaxation constants $\eta_{j}$ for the respective bands. The accompanying Maxwell equation writes as

$$
\frac{c}{4 \pi}[\nabla \times[\nabla \times \mathbf{A}]]=\mathbf{J}_{n}+\mathbf{J}_{s}
$$

where $\mathbf{J}_{s}$ is the supercurrent density

$$
\mathbf{J}_{s}=2 e \operatorname{Re}\left[\frac{1}{m_{1}} \Psi_{1} \mathbf{D}^{*} \Psi_{1}^{*}+\frac{1}{m_{2}} \Psi_{2} \mathbf{D}^{*} \Psi_{2}^{*}\right]
$$

and $\mathbf{J}_{n}$ is the normal current density induced by the electric field

$$
\mathbf{J}_{n}=\sigma \mathbf{E}=-\frac{\sigma}{c} \frac{\partial \mathbf{A}}{\partial t}
$$


with $\sigma$ being the normal conductivity of the material.

Although characteristic lengths of this model strongly depend on the coupling between the bands, one can still define the coherence and the penetration lengths for each band, considered separately, by using the standard GL expressions

$$
\xi_{j}=\frac{\hbar v_{j}}{\sqrt{6} W}, \quad \lambda_{j}=\sqrt{\frac{3 c^{2}}{16 \pi N(0) e^{2} n_{j} v_{j}^{2}}} .
$$

The critical temperatures of the uncoupled bands (that is, the temperatures at which the corresponding $\alpha_{j}$ changes sign) are given by the expression

$$
T_{c j}=T_{c}\left(1-\frac{S_{j}}{n_{j} G}\right)
$$

Notice that both $T_{c j}$ are always smaller than $T c$. There for, $T_{c 1}, T_{c 2}<T<T_{c}$, both $\alpha_{j}$ are positive and superconductivity survives in the system only due to coupling between the bands, where as for $T<T_{c 1}, T_{c 2}$, both bands are active $\left(\alpha_{j}<0\right)$. For temperatures such that $T_{c P}<T<T_{c A}$, one of the bands is active(band A) while the other (band $\mathrm{P}$ ) is passive, that is, it remains superconducting only because of Cooper pairs coming from band A.

One can also define the GL parameter $\kappa$ for each of the band as

$$
\kappa_{j}=\frac{\lambda_{j}}{\xi_{j}}, \quad \frac{\kappa_{2}}{\kappa_{1}}=\sqrt{\frac{n_{1}}{n_{2}}} \frac{v_{1}^{2}}{v_{2}^{2}}
$$

For the numerical calculations it is convenient to scale all relevant quantities using parameters of the stronger band $j=1$,

$$
\begin{aligned}
& \psi_{j}=W \tilde{\Psi}_{j}, \quad \mathbf{r}=\xi_{1} \tilde{\mathbf{r}}, \quad \mathbf{A}=A_{0} \tilde{\mathbf{A}} \\
& t=t_{0} \tilde{t}, \quad \eta_{j}=\eta_{0}^{j} \tilde{\eta}_{j},
\end{aligned}
$$

where

$$
A_{0}=\frac{\hbar c}{2 e \xi_{1}}, \quad t_{0}=\frac{4 \pi \sigma \kappa_{1}^{2} \xi_{1}^{2}}{c^{2}}, \quad \eta_{0}^{j}=n_{j} N(0) t_{0}
$$

The scaled TCGL equations read as (hereafter we omit "tilde" for the scaled quantities)

$$
\begin{aligned}
& \eta_{1} \frac{\partial \Psi_{1}}{\partial t}=\mathbf{D}^{2} \Psi_{1}-\left(\chi_{1}-\left|\Psi_{1}\right|^{2}\right) \Psi_{1}-\gamma \Psi_{2} \\
& \eta_{2} \frac{\partial \Psi_{2}}{\partial t}=\frac{1}{\alpha} \mathbf{D}^{2} \Psi_{2}-\left(\chi_{2}-\left|\Psi_{2}\right|^{2}\right) \Psi_{2}-\frac{\gamma}{\alpha} \frac{n_{1}}{n_{2}} \Psi_{1}
\end{aligned}
$$

where the gradient-invariant derivative is $\mathbf{D}=-i \nabla-\mathbf{A}, \gamma=\lambda_{12} /\left(n_{1} G\right)$ and for the decay 
rates we can assume $\eta_{1}=\eta_{2}$. Equation (2.9.5) becomes

$$
\frac{\partial \boldsymbol{A}}{\partial t}=\operatorname{Re}\left[\Psi_{1} \mathbf{D}^{*} \Psi_{1}^{*}+\frac{1}{\alpha} \frac{n_{2}}{n_{1}} \Psi_{2} \mathbf{D}^{*} \Psi_{2}^{*}\right]-\kappa_{1}^{2}[\nabla \times[\nabla \times \boldsymbol{A}]]
$$

2.10 Vortex dissociation in the London model

This section is based in the work of Shi-Zeng Lin and Lev N. Bulaevskii [34].

We will study the dissociation of a composite vortice in two-band superconductors by using the London Model.

The free energy density can be written as

$$
F_{L}=\frac{1}{8 \pi} \sum_{\mu=2}^{2}\left[\frac{1}{\lambda_{\mu}^{2}}\left(\mathbf{A}-\frac{\Phi_{0}}{2 \pi} \nabla \theta_{\mu}\right)^{2}+(\nabla \times \mathbf{A})^{2}\right]
$$

where $\lambda_{\mu}=\sqrt{\left(m_{\mu} c^{2}\right) /\left(4 \pi n_{\mu} e^{2}\right)}$ is the London penetration depth for each condensate with superfluid density $n_{\mu}, \mathbf{A}$ is vector potential, $m_{\mu}$ is the electron mass in $\mu$-th band and $\gamma$ is the interband Josephson coupling. The effective penetration depth for the two-band system is $\lambda^{-2}=\Sigma_{\mu=1}^{2} \lambda_{\mu}^{-2}$.

Minimizing $F_{L}$ with respect to $\mathbf{A}$, we obtain the London equation

$$
\lambda^{2} \nabla \times \nabla \times \mathbf{B}+\mathbf{B}=\Phi_{\mu} \sum_{\mu, j} \delta\left(\mathbf{r}-\mathbf{r}_{\mu, j}\right)
$$

with $\Phi_{\mu}=\lambda^{2} \Phi_{0} / \lambda_{\mu}^{2}$ the fractional quantum flux and $\mathbf{r}_{\mu, j}=\left(x_{\mu, j}, y_{\mu, j}\right)$ is the vortex coordinates for the vortex in the $\mu$-th condensate.

$F_{L}$ can be splited into two contribution [35] $F_{L}=F_{m}+F_{c}$. Where $F_{m}$ is the magnetic coupling

$$
F_{m}=\frac{1}{8 \pi}\left[\mathbf{B}^{2}+\lambda^{2}(\nabla \times \mathbf{B})^{2}\right]
$$

and $F_{c}$ is the coupling due to the phase difference between condensates

$$
F_{c}=\frac{\Phi_{1} \Phi_{2}}{32 \pi^{3} \lambda^{2}}\left[\nabla\left(\theta_{1}-\theta_{2}\right)\right]^{2}-\gamma \cos \left(\theta_{1}-\theta_{2}\right) .
$$

For a fractional vortex where $\theta_{1}$ changes by $2 \pi$ around $\mathbf{r}_{0}$ while $\theta_{2}$ does not change, the self energy per unit length is

$$
E_{f v}=\left(\frac{\Phi_{1}}{4 \pi \lambda}\right)^{2} \ln \left(\frac{\lambda}{\xi_{1}}\right)+\frac{\Phi_{1} \Phi_{2}}{16 \pi^{2} \lambda^{2}}\left(\frac{L}{\xi_{1}}\right)+|\gamma| \int d r^{2}\left[1-\cos \theta_{1}\right]
$$

where $L$ is the linear size of the system and $\xi_{\mu}$ is the coherence length. $E_{f, v}$ diverges at $L \rightarrow \infty$. Thus a fractional vortex is thermodynamically unstable in bulk superconductor. 
To calculate intraband and interband interaction, one need to know $\theta_{\mu}$. It can be obtained minimizing Eq. (2.10.4) with respect to $\theta_{1}$

$$
\frac{\Phi_{1} \Phi_{2}}{16 \pi^{3} \lambda^{2}} \nabla^{2}\left(\theta_{1}-\theta_{2}\right)-\gamma \sin \left(\theta_{1}-\theta_{2}\right)=0
$$

subject to the boundary condition accounting for vortices

$$
\nabla \times\left(\nabla \theta_{\mu}\right)=2 \pi \sum_{\mu, j} \delta\left(\mathbf{r}-\mathbf{r}_{\mu, j}\right) .
$$

It can be deduced from the Eqs. (2.10.6) and (2.10.7) that the interaction between vortices is a many-body interaction. For strong field the sin term in Eq.(2.10.6) becomes smaller than the Laplacian term, thus the first one can be neglected. The discussion of the interband and intraband interaction will be analized neglecting the Josephson interband coupling term. Consequently $F_{m}$ and $F_{c}$ are quadratic in $\mathbf{B}$ and $\theta_{\mu}$, so the interaction between vortices is pairwise. $F_{m}$ is responsible for short-range interband and intraband repulsion between vortices with the same polarization. $F_{c}$ is responsible for long range interaction.

Defining $\mathbf{r}_{\mu, i j} \equiv \mathbf{r}_{\mu, i}-\mathbf{r}_{\mu, j}$, the intraband repulsion between two vortices in the same condensate is

$$
V_{\text {intra }}\left(r_{\mu, i j}\right)=\frac{\Phi_{\mu}^{2}}{8 \pi^{2} \lambda^{2}} K_{0}\left(\frac{r_{\mu, i j}}{\lambda}\right)-\frac{\Phi_{1} \Phi_{2}}{8 \pi^{2} \lambda^{2}} \ln \left(r_{\mu, i j}\right)
$$

and defining $\mathbf{r}_{12, i j} \equiv \mathbf{r}_{1, i}-\mathbf{r}_{2, j}$, the interband attraction between two vortices in the different condensates is

$$
V_{\text {inter }}\left(r_{12, i j}\right)=\frac{\Phi_{1} \Phi_{2}}{8 \pi^{2} \lambda^{2}}\left[K_{0}\left(\frac{r_{12, i j}}{\lambda}\right)+\ln \left(r_{12, i j}\right)\right] .
$$

The two last equations are valid away from vortex cores.

When external current is applied in the superconductor, the vortices move due to the Lorentz force. The vortex in each band experiment a viscosity given by the BardeenStephen model $\eta_{\mu}=\Phi_{0}^{2} /\left(2 \pi c^{2} \xi_{\mu}^{2}\right)$. The dynamic of vortices can be expressed by the follow equations

$$
\begin{aligned}
\eta_{\mu} \partial_{t} r_{\mu, i}=\frac{1}{8 \pi^{2} \lambda^{3}} \sum_{j}\left[\Phi_{\mu}^{2} K_{1}\left(\frac{r_{\mu, i}}{\lambda}\right)\right. & \left.+\frac{\Phi_{1} \Phi_{2} \lambda}{r_{\mu, i j}}\right] \\
& +\frac{\Phi_{1} \Phi_{2}}{8 \pi^{2} \lambda^{3}} \sum_{j}\left[K_{1}\left(\frac{r_{12, i j}}{\lambda}\right)-\frac{\lambda}{r_{12, i j}}\right]+\frac{J \Phi_{\mu}}{c}
\end{aligned}
$$

Considering a square lattice and a approximation $2 \pi \lambda / a \gg 1$, the equation of motion for 
the center of mass of vortex lattice $R_{\mu}$ in each band becomes

$$
\begin{gathered}
\eta_{2}^{\prime} \partial_{t}\left(R_{2}-R_{1}\right)=-\left(1+\eta_{2}^{\prime}\right) \sin \left(R_{2}-R_{1}\right)+\left(\Phi_{2}^{\prime}-\eta_{2}^{\prime}\right) J \\
\partial_{t} R_{1}+\eta_{2}^{\prime} \partial_{t} R_{2}=\left(1+\Phi_{2}^{\prime}\right) J
\end{gathered}
$$

The equation (2.10.11) are in dimensionless units: length is in unit of $a /(2 \pi)$, time in unit of $\eta_{1} a /\left(2 \pi F_{d}\right)$, current is in unit of $c F_{d} / \Phi_{1}, F_{d}$ is the maximum attractive force between two lattices $F_{d}=\Phi_{1} \Psi_{2} a /\left(64 \pi^{6} \lambda^{4}\right)$. $\Phi_{2}^{\prime} \equiv \Phi_{2} / \Phi_{1}$ and $\eta_{2}^{\prime} \equiv \eta_{2} / \eta_{1}$.

For small current, vortices in different bands move with the same velocity $v_{1}=v_{2}=$ $\left(1+\eta_{2}^{\prime}\right)^{-1} \times\left(1+\Phi_{2}^{\prime}\right) J$. However the center of mass of this lattices vortex are deviated with a separation $\sin ^{-1}\left[\left(1+\eta_{2}^{\prime}\right)^{-1}\left(\Phi_{2}^{\prime}+\eta_{2}^{\prime}\right)^{-1} J\right]$.

The maximum attraction is reached at $R_{2}-R_{1}=\pi / 2$ or $a / 4$ in real unit. At a threshold current

$$
J_{d}=\left|\left[\left(1+\eta_{2}^{\prime}\right)^{-1}\left(\Phi_{2}^{\prime}+\eta_{2}^{\prime}\right)^{-1} J\right]\right|
$$

the vortices dissociate and move with velocity

$$
\left.v_{\mu}=\left(1+\eta_{2}^{\prime}\right)^{-1} \times\left[1+\Phi_{2}^{\prime}\right) J-\frac{\eta_{1}}{\eta_{\mu}} \sqrt{\left(\Phi_{2}^{\prime}-\eta_{2}^{\prime}\right)^{2} J^{2}-\left(1+\eta_{2}^{\prime}\right)^{2}}\right]
$$

The Fig. 5 show the dependence of $v_{\mu}$ on $J$. For current $J \gg J_{d}$, each lattice move independently with velocities $v_{1}=J$ and $v_{2}=J / \eta_{2}^{\prime}$.

The $I$ - $V$ behavior is calculated by the expression $\eta_{1} v_{1}^{2}+\eta_{2} v_{2}^{2}=J E a^{2}$. The characteristic $I$ - $V$ curve is shown in $\mathrm{vv}$ and it can be observed that the differential resistivity increases in the decoupled phase.

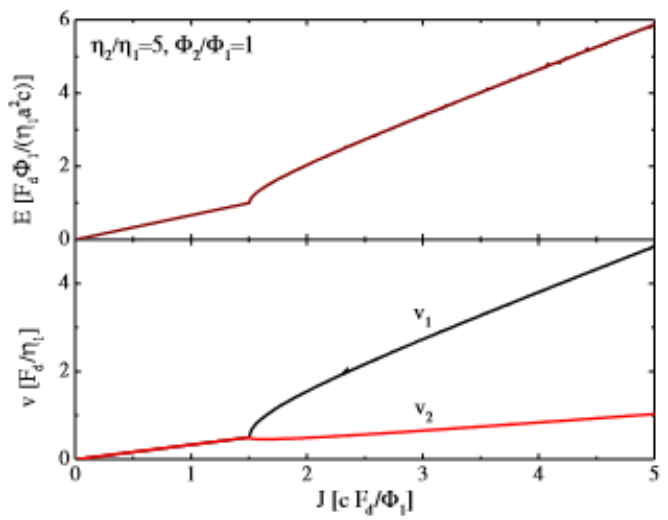

Figure 5: The dependence of the electric field (a) and of the velocities $v_{1}, v_{2}$ (b)on the current density $J$. 


\section{Numerical method}

\section{$3.1 \psi$-U method}

The $\psi$-U method will be applied to discretize the two-component Ginzburg-Landau model. We first will discretize the time dependent Ginzburg Landau (TDGL) equations for single band. This method is described in detail in Ref. [108].

\subsubsection{Discretization of time dependent Ginzburg-Landau equations}

The TDGL equations were deduced in the framework of BCS theory by L.P Gor'kov and G.M. Ėliashberg [109]. The TDGL equations coupled with Maxwell equations with the zero-scalar potential gauge are written in dimensionless form as

$$
\begin{gathered}
\partial_{t} \Psi=-\frac{1}{\eta}\left[(-i \nabla-\mathbf{A})^{2} \Psi+(1-T)\left(|\Psi|^{2}-1\right) \Psi\right], \\
\partial_{t} \mathbf{A}=(1-T) \operatorname{Re}[\bar{\Psi}(-i \nabla-\mathbf{A}) \Psi]-\kappa^{2} \nabla \times \nabla \times \mathbf{A},
\end{gathered}
$$

where lengths have been scaled in units of $\xi(0)$, time in units of $t_{0}=\pi \hbar /\left(96 k_{B} T_{c}\right), \mathbf{A}$ in units of $H_{c 2}(0)$ and temperature in unit of $T_{c}$.

We start introducing the auxiliary fields

$$
\begin{aligned}
& \mathscr{U}^{x}(x, y)=\exp \left(-i \int_{x_{0}}^{x} A^{x}(x \prime, y) d x \prime\right) \\
& \mathscr{U}^{y}(x, y)=\exp \left(-i \int_{y_{0}}^{y} A^{y}\left(x, y^{\prime}\right) d y \prime\right)
\end{aligned}
$$

which preserve the gauge invariance of the TDGL equations. $\left(x_{0}, y_{0}\right)$ is an arbitrary point. The discretization of the TDGL are done in a rectangular mesh consisting of $N_{x} \times N_{y}$ cell points. The lattice spacing between adjacent cell points is $\left(a_{x}, a_{y}\right)$. An arbitrary vertex point in the mesh is denoted by $\left(x_{i}, y_{i}\right)$.

The TDGL equations will be written in term of follow unknowns variables: 
- $\Psi_{i, j}$, with $1 \leq i \leq N_{x}+1,1 \leq j \leq N_{y}+1$. This is named vertex variable, so it is associated to the nodes.

- $U_{i, j}^{x}$, with $1 \leq i \leq N_{x}, 1 \leq j \leq N_{y}+1$. This is named link variable in the $x$ direction. It is associated to the horizontal cell edges of the mesh.

- $U_{i, j}^{y}$, with $1 \leq i \leq N_{x}+1,1 \leq j \leq N_{y}$. This is named link variable in the $y$ direction. It is associated to the vertical cell edges of the mesh.

The Fig. 6 show a mesh defining the numbering of discrete variables

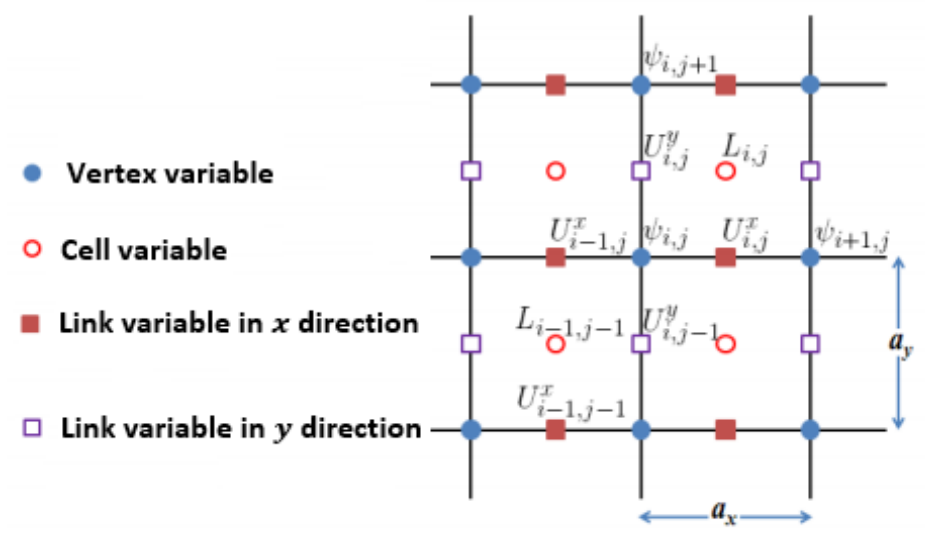

Figure 6: Sketch of cells defining the numbering of discrete variables.

The quantities defined by Eqs. (3.1.3) and (3.1.4) can be discretized as follow

$$
\mathscr{U}_{i, j}^{x}=\prod_{k=1}^{i-1} U_{k, j}^{x}, \quad \mathscr{U}_{i, j}^{y}=\prod_{k=1}^{j-1} U_{k, j}^{y}
$$

leading to

$$
U_{i, j}^{x}=\overline{\mathscr{U}}_{i, j}^{x} \mathscr{U}_{i+1, j}^{x}, \quad U_{i, j}^{y}=\overline{\mathscr{U}}_{i, j}^{y} \mathscr{U}_{i, j+1}^{y}
$$

Now we will discretize each term of the TDGL:

- Term $(-i \nabla-\mathbf{A})^{2} \Psi$ : Using the identity

$$
(-i \nabla-\mathbf{A})^{2} \Psi=-\overline{\mathscr{U}}^{x} \partial_{x x}^{2}\left(\mathscr{U}^{x} \Psi\right)-\overline{\mathscr{U}}^{y} \partial_{y y}^{2}\left(\mathscr{U}^{y} \Psi\right)
$$

a second order approximation at $\left(x_{i}, y_{i}\right)$ reads

$$
\begin{aligned}
&\left.(-i \nabla-\mathbf{A})^{2} \Psi\right|_{\left(x_{i}, y_{j}\right)}=-\frac{U_{i, j}^{x} \Psi_{i+1, j}-2 \Psi_{i, j}+\bar{U}_{i-1, j}^{x} \Psi_{i-1, j}}{a_{x}^{2}} \\
&-\frac{U_{i, j}^{y} \Psi_{i, j+1}-2 \Psi_{i, j}+\bar{U}_{i, j-1}^{y} \Psi_{i, j-1}}{a_{y}^{2}}+\ldots
\end{aligned}
$$


- The term $\left(|\Psi|^{2}-1\right) \Psi$ is immediately approximated by

$$
\left.\left(|\Psi|^{2}-1\right) \Psi\right|_{\left(x_{i}, y_{j}\right)}=\left(\bar{\Psi}_{i, j} \Psi_{i, j}-1\right) \Psi_{i, j}
$$

- The term $\operatorname{Re}[\bar{\Psi}(-i \nabla-\mathbf{A}) \Psi]$ : Using the identity

$$
\left(-i \partial_{x}-A_{x}\right) \Psi=-i \overline{\mathscr{U}}^{x} \partial_{x}\left(\mathscr{U}^{x} \Psi\right)
$$

we obtain that

$$
\begin{array}{r}
\left.\operatorname{Re}\left[\bar{\Psi}\left(-i \partial_{x}-A_{x}\right) \Psi\right]\right|_{x_{i}+\frac{a_{x}}{2}, y_{j}} \\
=\operatorname{Im}\left(\frac{\overline{\mathscr{U}}_{i, j}^{x} \bar{\Psi}_{i, j}+\overline{\mathscr{U}}_{i+1, j}^{x} \bar{\Psi}_{i+1, j}}{2} \frac{\mathscr{U}_{i+1, j}^{x} \Psi_{i+1, j}-\mathscr{U}_{i, j}^{x} \Psi_{i, j}}{a_{x}}\right)+\ldots \\
=\frac{1}{a_{x}} \operatorname{Im}\left(\bar{\Psi}_{i, j} \overline{\mathscr{U}}_{i, j}^{x} \mathscr{U}_{i+1, j}^{x} \Psi_{i+1, j}\right)+\ldots=\frac{1}{a_{x}} \operatorname{Im}\left(\bar{\Psi}_{i, j} U_{i, j}^{x} \Psi_{i+1, j}\right)+\ldots
\end{array}
$$

and analogously for the y component.

- The term $\nabla \times \nabla \times \mathbf{A}=\nabla \times \mathbf{B}$ : First, the follow auxiliary variables are introduced

$$
L_{i, j}=U_{i, j}^{x} U_{i+1, j}^{y} \bar{U}_{i, j+1}^{x} \bar{U}_{i, j}^{y}
$$

and by using the Stokes' identity it is obtained

$$
L_{i, j}=\exp \left(-i a_{x} a_{y} B_{z}\left(x_{i}+\frac{a_{x}}{2}, y_{j}+\frac{a_{y}}{2}\right)\right)+\ldots
$$

We have considered a magnetic field in a $z$ direction $\mathbf{B}=\left(0,0, B_{z}\right)$

$$
\begin{gathered}
\nabla \times \mathbf{B}=\left(\partial_{y} B_{z},-\partial_{x} B_{z}, 0\right) \\
\partial_{y} B_{z}\left(x_{i}+\frac{a_{x}}{2}, y_{j}\right)=\frac{i}{a_{x} a_{y}^{2}}\left(\bar{L}_{i, j-1} L_{i, j}-1\right)+\ldots \\
-\partial_{x} B_{z}\left(x_{i}, y_{j}++\frac{a_{y}}{2}\right)=\frac{i}{a_{x} a_{y}^{2}}\left(\bar{L}_{i, j} L_{i-1, j}-1\right)+\ldots
\end{gathered}
$$


- The term $\partial_{t} \mathbf{A}$ : We start from the equality

$$
\begin{aligned}
\partial_{t}\left[\overline{\mathscr{U}}^{x}(x, y, z) \mathscr{U}^{x}(x+\delta, y, t)\right] \\
=-i \overline{\mathscr{U}}^{x}(x, y, z) \mathscr{U}^{x}(x+\delta, y, t) \int_{x}^{x+\delta} \partial_{t} A_{x}(\xi, y, t) d \xi \\
=-i \delta \overline{\mathscr{U}}^{x}(x, y, t) \mathscr{U}^{x}(x+\delta, y, t) \partial_{t} A_{x}\left(x+\frac{\delta}{2}, y, t\right)+\ldots
\end{aligned}
$$

and consequently

$$
\partial_{t} A_{x}\left(x_{i}+\frac{a_{x}}{2}, y_{j}, t\right)=\frac{i}{a_{x}} \bar{U}_{i, j}^{x} \partial_{t} U_{i, j}^{x}+\ldots
$$

and the same for the $y$ component.

Finally the discretized TDGL equation for single bands are

$$
\begin{gathered}
\partial_{t} \Psi_{i, j}=\frac{U_{i, j}^{x} \Psi_{i+1, j}-2 \Psi_{i, j}+\bar{U}_{i-1, j}^{x} \Psi_{i-1, j}}{\eta a_{x}^{2}} \\
+\frac{U_{i, j}^{y} \Psi_{i, j+1}-2 \Psi_{i, j}+\bar{U}_{i, j-1}^{y} \Psi_{i, j-1}}{\eta a_{y}^{2}} \\
-\frac{1-T}{\eta}\left(\bar{\Psi}_{i, j} \Psi_{i, j}-1\right) \Psi_{i, j} \\
\partial_{t} U_{i, j}^{x}=-i(1-T) U_{i, j}^{x} \operatorname{Im}\left(\bar{\Psi}_{i, j} U_{i, j}^{x} \Psi_{i+1, j}\right)-\frac{\kappa^{2}}{a_{y}^{2}} U_{i, j}^{x}\left(\bar{L}_{i, j-1} L_{i, j}-1\right) \\
\partial_{t} U_{i, j}^{y}=-i(1-T) U_{i, j}^{y} \operatorname{Im}\left(\bar{\Psi}_{i, j} U_{i, j}^{y} \Psi_{i, j+1}\right)-\frac{\kappa^{2}}{a_{x}^{2}} U_{i, j}^{y}\left(\bar{L}_{i, j} L_{i-1, j}-1\right)
\end{gathered}
$$

For discretizing the time we use the forward-Euler scheme with step $\Delta t$

$$
\Psi_{i, j}(t+\Delta t)=\Psi_{i, j}(t)+\Delta t \partial_{t} \Psi_{i, j}(t)
$$

and analogously for $U_{i, j}^{x}$ and $U_{i, j}^{y}$.

For the applicability of the Euler step, the condition

$$
\Delta t \leqslant \min \left(\frac{\sigma^{2} \eta}{4}, \frac{\sigma^{2}}{4 \kappa^{2}}\right)
$$


with

$$
\sigma^{2}=\frac{2}{a_{x}^{-2}+a_{y}^{-2}}
$$

must be satisfied.

\section{External boundary conditions}

The Eq.s (3.1.19)-(3.1.21) are not defined for boundary nodes or links.

The condition $\left.\widehat{\mathbf{n}} \cdot(-i \nabla-\mathbf{A}) \Psi\right|_{s}=0$ applied in surface boundary aligned with $y$ lead

$$
\begin{gathered}
\Psi_{1, j}=U_{1, j}^{x} \Psi_{2, j} \\
\Psi_{N_{x}+1, j}=\bar{U}_{N_{x}, j}^{x} \Psi_{N_{x}, j}
\end{gathered}
$$

Now, we applied the same condition, but in surface boundary aligned with $x$,

$$
\begin{gathered}
\Psi_{i, 1}=U_{i, 1}^{y} \Psi_{i, 2} \\
\Psi_{i, N_{y}+1}=\bar{U}_{i, N_{y}}^{y} \Psi_{i, N_{y}}
\end{gathered}
$$

If an external magnetic field $\mathbf{H}_{a}$ is applied, the condition at the cell of the boundary give

$$
L_{i, j}=U_{i, j}^{x} U_{i+1, j}^{y} \bar{U}_{i, j+1}^{x} \bar{U}_{i, j}^{y}=\exp \left(-i a_{x} a_{y} H_{e}\right)
$$

\subsubsection{Discretization of two-component Ginzburg-Landau (TCGL) equations}

The discretization of TCGL equations is a simple generalization of the single band case. Using Eqs. (3.1.8) - (3.1.18) we obtain the discretized TCGL equations

$$
\begin{aligned}
& \eta_{1} \partial_{t} \Psi_{i, j}^{(1)}=\frac{U_{i, j}^{x} \Psi_{i+1, j}^{(1)}-2 \Psi_{i, j}^{(1)}+\bar{U}_{i-1, j}^{x} \Psi_{i-1, j}^{(1)}}{a_{x}^{2}} \\
&+\frac{U_{i, j}^{y} \Psi_{i, j+1}^{(1)}-2 \Psi_{i, j}^{(1)}}{a_{y}^{2}}+\bar{U}_{i, j-1}^{y} \Psi_{i, j-1}^{(1)} \\
& \quad-\left(\chi_{1}-\bar{\Psi}_{i, j}^{(1)} \Psi_{i, j}^{(1)}\right) \Psi_{i, j}^{(1)}-\gamma \Psi^{(2)}
\end{aligned}
$$




$$
\begin{aligned}
\eta_{2} \partial_{t} \Psi_{i, j}^{(2)}=\frac{U_{i, j}^{x} \Psi_{i+1, j}^{(2)}-2 \Psi_{i, j}^{(2)}+\bar{U}_{i-1, j}^{x} \Psi_{i-1, j}^{(2)}}{\alpha a_{x}^{2}} & \\
+\frac{U_{i, j}^{y} \Psi_{i, j+1}^{(2)}-2 \Psi_{i, j}^{(2)}+\bar{U}_{i, j-1}^{y} \Psi_{i, j-1}^{(2)}}{\alpha a_{y}^{2}} & -\left(\chi_{2}-\bar{\Psi}_{i, j}^{(2)} \Psi_{i, j}^{(2)}\right) \Psi_{i, j}^{(2)}-\frac{\gamma n_{1}}{\alpha n_{2}} \Psi^{(1)}
\end{aligned}
$$

For discretizing the time we use the forward-Euler scheme with step $\Delta t$

$$
\Psi_{i, j}^{\mu}(t+\Delta t)=\Psi_{i, j}^{\mu}(t)+\Delta t \partial_{t} \Psi_{i, j}^{\mu}(t)
$$

where $\mu=1,2$ is the band index

For obtaining the expressions of the auxiliary fields $U_{i, j}^{x}$ and $U_{i, j}^{y}$ the procedure is similar. The boundary conditions for two bands component are equal for the case of single bands, but applied on each band separately.

\subsubsection{Computational details}

The discretized TCGL equations were solved by iteration method through the computational program written in CUDA C.

The system was initialized in the normal state.

For applying the Euler method we selected a time step, $\Delta t=0.0025$, that is used to ensure stability [108].

We took a 1,600.000 iterations for reaching the equilibrium state at fixed external magnetic field and absence of current density. Further we apply an increasing current density from zero to some finite value, using an increment, $d j=0.0001$. For each value of density current we employed 4,000.000 iterations in order to reach the no transient dynamical state. After all iterations we save for each current density the files for order parameters and the voltage induced by the vortex movement. 


\section{Vortex dissociation in two-band superconductors}

We use the TCGL model to identify and analyze the non-composite vortex state in a superconducting slab in a presence of an applied constant external magnetic field $(\mathbf{H})$ and current density (j) (hereafter briefly named current). We describe through the $\mathbf{H}-\mathbf{j}$ diagram, the values of external magnetic field and current at which the composite vortex lattice are dissociated, showing which states bound the non-composite vortex state. We study the influence of the rates of entying vortices and the presence of fractional vortices in the dissociation process, besides the difference between interband vortex velocities. The roll of the strength of the Josephson interband coupling is also studied. It is presented the Pearson product-moment correlation coefficient $(r)$ between the order parameters of the bands as mathematics tool for detecting the presence of this non-composite vortex state. Finally it is exposed how the non-composite vortex state affect the resistance in the superconductor.

\subsection{Introduction}

In superconductors materials, vortices can be seen as elementary entities, serving as building blocks for all non-trivial phase configurations in 2D structures. Multi-band superconductors can develop vortex states that are qualitatively different from those in single-band materials. A trivial configuration is a composite vortex, that comprises two vortices in the band condensates, that are centred at the same point and have equal winding numbers $(n)$. Stationary equilibrium states in two-band bulk superconductors are formed by the composite vortices. However, an non-equilibrium system can develop a state with the band vortices that are shifted spatially, the so-called non-composite vortices. Those vortices are associated with a non-quantised magnetic flux: each of the partial vortices in the band condensates carry a fraction of the total (quantised) flux, and are often called fractional vortices. In bulk superconductors fractional vortex states are thermodynamically inestable due to a divergent energy. [12]. However, in samples of finite dimensions metastable fractional vortices can appear [35-38,40,110]. One of the mechanism which creates non-composite fractional vortices is the dynamical vortex dissociation. This mechanism appears, for example, when a superconducting current flows through the sample. 
In this case differences in the driving forces and the viscosity of the vortex matter in each of the condensates drives the band vortices apart. [34].

\subsection{Model and Method}

The model system considered was a two-band superconducting slab with infinite dimensions in $z$ and $x$ axis and finite dimension in $y$ direction as is show in Fig 7 .

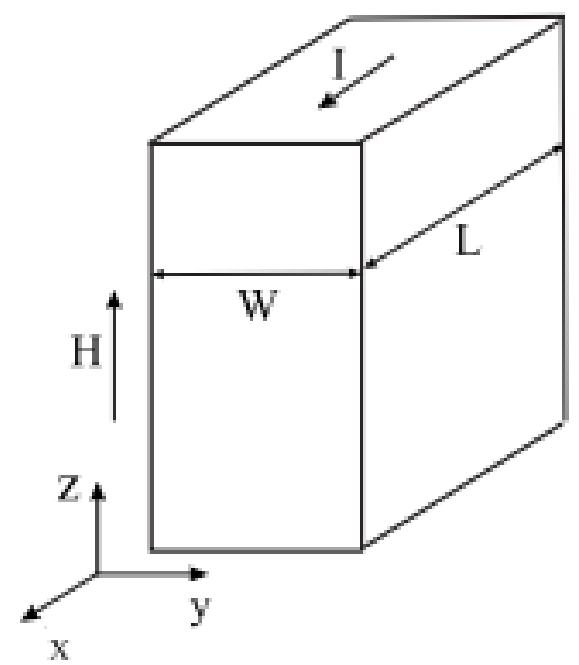

Figure 7: Superconducting slab with infinite dimensions in $z$ and $x$ axis and finite in $y$ direction. A magnetic field $(H)$ is applied parallel at $z$ and current $(I)$ along the $x$ direction.

The system is immersed in a magnetic field in $z$ direction and an increasing current $(I)$ is applied along of $x$ axis. We neglect the possibility of the formation of curved vortices in $z$ direction and the study may be performed in two-dimensional system. The confinement effects is also neglect because the width is much larger than the field penetration depth.

The numerical simulation is developed in the framework of TCGL theory (section 2.9). In order to preserve the validity of the model, the individual band critical temperatures (Tci) were chosen to be close to the superconducting critical temperature of the specimen. The TCGL equations were integrated on a two-dimensional squared grid with spacings $a_{x}=a_{y}=\xi_{1}$, using the $U-\Psi$ method (section 3.1) combined with a stable semi-implicit algorithm [111] for the time evolution. For the boundary conditions, periodic ones were used along the $x$ direction $[\Psi(x)=\Psi(x+L)$ and $\mathbf{A}(x)=\mathbf{A}(x+L)]$ and Neumann boundary conditions, $\left.(\nabla-i \vec{A}) \psi_{j}\right|_{n}=0$, was set in the $y$ direction. The vortex dynamics is induced by the current density, $j$, introduced by the boundary conditions for $\nabla \times \vec{A}$ at the interfaces perpendicular to $y$, located at $y=0$ and $y=W$. For $\left.(\nabla \times \vec{A})\right|_{y=0}=H-\Delta H$ and $\left.(\nabla \times \vec{A})\right|_{y=W}=H+\Delta H$ the expected current density is $j=c \Delta H / 2 \pi$. As an indicative of vortex movement from a surface (at $y=W$ ) towards the other one (at $y=0$ ), we have performed voltage, $V$, calculations along the $x$ direction. The current density $(j)$ and voltage $(V)$ were scaled, respectively, by the critical current density $\left(j_{0}\right)$ and voltage 
$\left(V_{0}\right)$ that set the point in which the normal state is completely reached. The external magnetic field is scaled in term of the thermodynamical critical field $H_{c}$.

For our numerical simulation we took $\kappa_{1}=10.0, T=0.85 T_{c}$. We ensure that $T_{c 1}$ and $T_{c 2}$ are close to $T_{c}$ choosing $\lambda_{11} \approx 2 \lambda_{22}, \lambda_{11}=2.0, \lambda_{22}=1.03$. Using these parameters one can calculate the critical temperatures for each of the band, taken separately, by Eq. (2.9.9), which yields $T_{c 1}=0.9997 T_{c}$ and $T_{c 2}=0.9030 T_{c}$. As the non-composite vortex states have more probability of appear at weak Josephson coupling between the bands $[38,40,110]$, we took $\lambda_{12}=0.0001 . v_{1} / v_{2}=0.52, n_{1}=0.3554, \eta=5.0$. This set of parameter were maintened constant in the simulation.

\subsection{Phases diagram $(\mathrm{H}-\mathrm{j})$ of vortex lattice configurations}

The diagram H-j (Fig. 8.a) show several vortex lattice states as a function of applied magnetic field $(\mathbf{H})$ and current density $(\mathbf{j})$. The slab dimensions were $W=150, L=300$. We named these states by phases $I, I I, I I I, I V$ and $V$. This phases start at $j=0, j=$ $J_{I I}, j=J_{I I I}, j=J_{I V}, j=J_{V}$ respectively. We have special interest and will emphasize in the state where the non-composite vortices appear (phase $I I I$ ). We are also interested in determine which phases bounds non-composite vortex state.

For describing each phase we selected states $(b, c, d, e, f, g, h, i, j, k, m$ and $n)$ along the three vertical dotted lines in the diagram. This dotted lines correspond to the fields, $H=2.4 H_{c}, H=7.3 H_{c}$ and $H=1.13 H_{c}$ respectively.

We introduce a reference magnetic field $H_{p}$ as the field at which the vortices start to penetrated the weaker band in absent of an applied current density. For our parameters $H_{p} \sim 0.65 H_{c}$. Then the fields of dotted lines selected in the diagram are: $H=2.4 H_{c}<$ $H_{p}, H=7.3 H_{c} \sim H_{p}$ and $H=1.13 H_{c}>H_{p}$. The classification of fields in reference of $H_{p}$ will be useful for organization of our results.

The fractional vortices with winding number $n_{1}=0$ and $n_{2}=1$ are present in all phases for all magnetic field (Fig. 8.b - 8.n ). This type of fractional vortices find their equilibrium positions near the surface in a similar fashion to those reported in $[35,36]$. The derivation for Bean-Livingston barrier suppression done in [112] was modified in [35] for taking into consideration the characteristic of a two band superconductor. Analytical expression for the critical value of magnetic field suppressing the surface barrier was found to be

$$
H_{s}=\frac{\Phi_{0}}{4 \pi \lambda \xi}
$$

For the parameters used here, here $\xi_{2}>\xi_{1}$, so $H_{s 2}<H_{s 1}$ and the vortices enter first in the second (weaker) band.

We start our analysis with $H=0.73 \sim H_{p}$, because for this field the vortex lattices present the five phases $(I, I I, I I I, I V$ and $V)$. The states $f, g, h, i$ and $j$ on the second 
vertical dotted line of diagram correspond to this field. We follow describing each phase: Phase $I$ : The vortex lattice is stationary. Initially we have vortices only in one band and further when the current is increased both bands are penetrated. The composite vortices are present in whole the sample. For a large number of vortices are present in the sample, they are arranged as a stationary quasi perfect Abrikosov lattice.

Phase $I I$ : The threshold current of this phase is the current at which the flux flow regime start $\left(j=J_{I I}\right)$. More vortices have penetrated the sample. The composite vortices fill the central region of the slab. The Abrikosov vortex lattice is maintained (Fig. 8.f), but now it is moving.
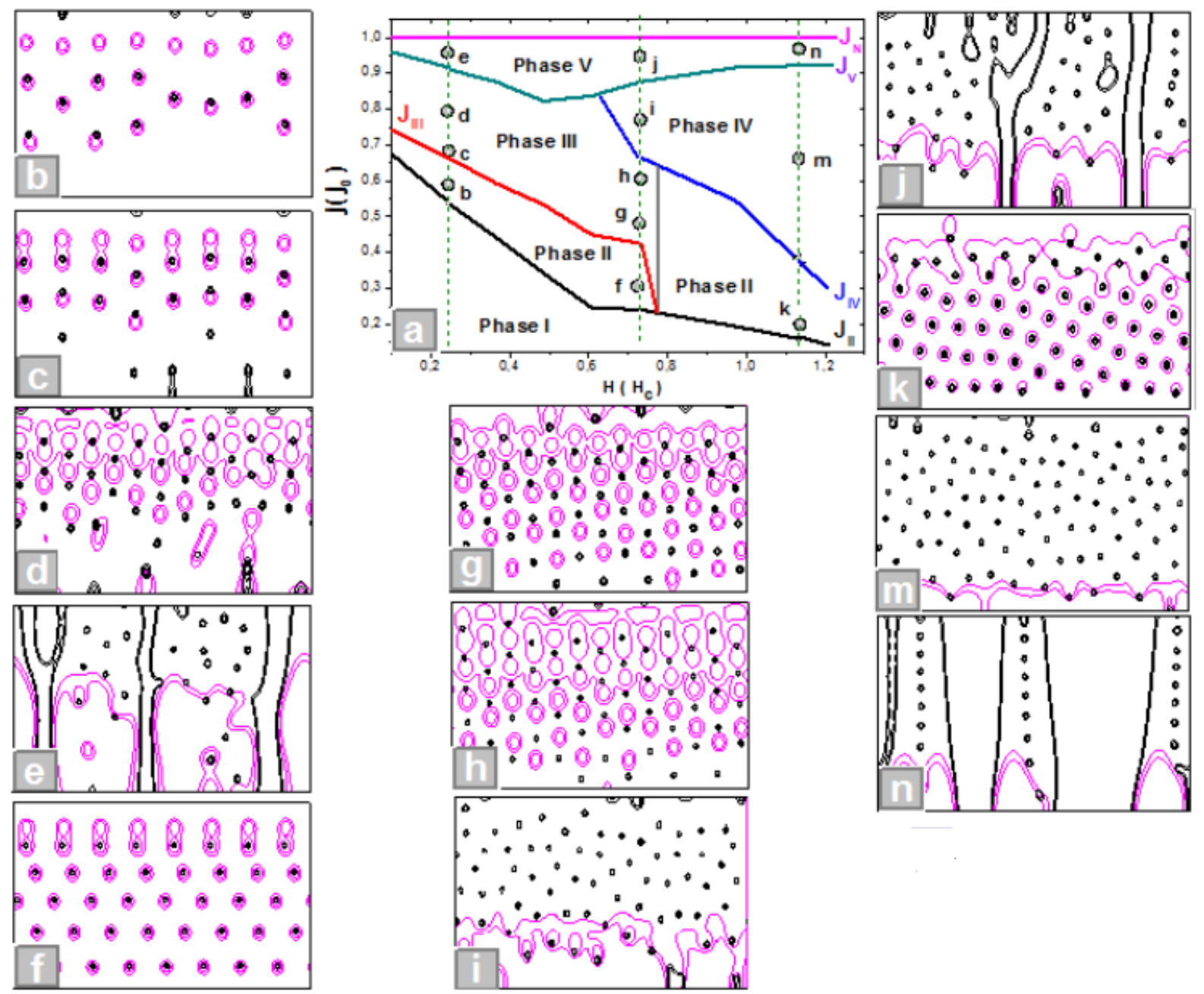

Figure 8: (a) Phase diagram $(\mathbf{H}-\mathbf{j})$ of vortex lattice states for $W=150, L=300$. The state of dissociated vortex is represented by phase $I I I$. (b)-(n) Vortex configurations corresponding to selected states $b, c, d, e, f, g, h, i, j, k, m$ and $n$, respectively from (a). The vortices in the first (second) band are represented by black (magenta) balls. The three vertical green dotted lines correspond to the fields, $H=0.24 H_{c}, H=0.73 H_{c}$ and $H=1.13 H_{c}$ respectively. For $H>H_{p}$ (and not comparable with $H_{p} \sim 0.65 H_{c}$ ) the phase of non-composite vortex (phase $I I I$ ) is absent, since the second band reaches the normal state before the composite vortices are dissociated, whereas the first (strong) band is still active (see $(\mathrm{m}))$.

Phase III: Above a threshold current $J_{I I I}$ the composite vortex lattices start to dissociate (Fig. 8.g - 8.h) [34]. $J_{I I I}$ was selected as value of $j$ at which the vortex lattices of two 
band move at different velocities and this will be studied in the next section.

Phase $I V$ : This phase is characterized by the vortices of the first band immersed in a normal background of the second band (Fig. 8.i). At $J_{I V}$ the second band is $60 \%$ in the normal state.

Phase $V$ : This phase start in the current, $J_{V}$, at which the continuous nonlinear branch in the $V(j)$ curve ends and a very high differential resistivity branch or a metastable branch is followed before transition to the normal state is achieved (diagramatesis.j). For $J>J_{V}$ both bands are mostly in the normal state.

At $j=J_{I I}$ the vortices in each condensates start to move driven by the Lorentz force. By our set of parameter, the fraction of the quantum flux $\Phi_{0}$ carried by the vortex in each band are respectively $\Phi_{1}=0.28 \Phi_{0}$ and $\Phi_{2}=0.72 \Phi_{0}$. Then the vortices in the second band experiment stronger force for carrying larger magnetic flux. Besides, the viscosity of vortex in different bands is different because these vortices have different coherence lenghs $\left(\xi_{2}=2.24 \xi_{1}\right)$. We have considered the Bardeen-Stephen model where the viscosity is given by $\eta_{\mu}=\Phi_{0}^{2} /\left(2 \pi c^{2} \xi_{\mu}^{2}\right)$. In this condition the vortices in the second band move faster then those in the first band. For small current these difference (force and viscosity) are compensed by the interband vortex attraction due to the coupling to the same gauge field. For current larger than $J_{I I I}$ this attraction is surpassed by the Lorentz force and composite vortices are dissociated. Although this dissociation was observed by Lin and Bulaevskii [34], they did not establish any restriction in the magnetic field for the observation of this effect. As can be observed in Fig. 8.a the states of non-composites vortices is present only for some values of $H$. The phase $I I I$ not exist for $H>H_{p}$. For understanding this behavior we look in greater detail for the states $k, m$ and $n$ on the third vertical line $\left(H=1.13 H_{c}\right)$ of the diagram. We observe that for a state for lower current (Fig. 8.k) and we observe that the vorticities, defined as $L_{\mu}$ for band $\mu=1$ and $\mu=2$, in the two bands are large and $L_{2}>L_{1}$ because the presence of fractional vortices in the second band, that are absent in the first one. In the $k$ state of second band there is a considerable normal region $\left(\Psi_{2} \approx 0\right)$ on the top of the slab. This normal region increases with the increasing current and the majority of second band reach the normal state before the composite vortices are dissociated (Fig. 8.m). For this reason the non-composite vortex state is not present for $H>H_{p}$. For illustrating this point we plotted the ratio of the normal state to total area for each band as a function of the current density for $H=1.13 H_{c}$ (Fig. 9.c) and it observed that the second band reaches the normal state much earlier than the first band. In can be seen in Fig 9.b that for $H=0.73 H_{c} \sim H_{p}$ the value of $J_{I V}$ is larger than the value for $H=1.13 H_{c}$. In general $J_{I V}$ decreases with the increasing $H$ not favouring the appearance of a dissociated vortex state. We emphasize that $J_{I I}, J_{I I I}$ and $J_{I V}$ decrease with $\mathrm{H}$ increasing (Fig. 8.a). This is due to the fact that for increasing $H$, the Bean-Livingston barrier is suppressed at smaller currents and consequently the flux-flow regime start much earlier. 
For $H<H_{p}$ the phase IV is absent and the state of dissociated vortex in the diagram is bounded by the phase $\mathrm{V}$, because the two bands reach the normal state simultaneously, as is illustrated in Fig. 8.e. Nevertheless, the weaker band (second band) allways reaches the normal state in advance of the stronger band (first band). The first dotted line in the diagram illustrate various states $\left(b, c, c\right.$ and $e$ ) for $H=0.24 H_{c}$. Since the applied field $\mathrm{H}$ is small, these are vorticity states. In the phase $I I$ there are not enough vortices for conforming the Abrikosov lattice, but this lattice is slightly deformed due to the high values of $J_{I I}$ (Fig. 8.b). The values of $J_{I I}, J_{I I I}$ and $J_{V}$ are high, because for low fields the Bean-Livingston barrier is suppressed at higher currents. There is a jump from phase $I I I$ to phase $V$ without passing by phase $I V$, because the fractional vortices with $n_{2}=1$ and $n_{1}=0$ have small contribution to reach the normal state and on the final of phase III the current is so high leading the two bands reaching the normal state simultaneously Fig. 9.a.

We emphasize that the presence of fractional vortices makes the weaker band to reach the normal state first than the strongest band. On the other hand high currents lead the two bands reach the normal state too. When $H<H_{p}$ the vorticity is small and fractional vortices have small contribution for the second band for reaching the normal state, whereas the high currents makes the two bands reach the normal state simultaneously. This explain, why for $H<H_{p}$ the non-composite vortices state are bounded by phase $V$ and the phase $I V$ is absent. When $H>H_{p}$ the second band reaches the normal state a lower currents because the vorticity in this band is high, whereas, the first band reaches the normal state with predominant influence of high current. Then we have only one active band and is not possible observe dissociation of vortices. For $H \sim H_{p}$ the fractional vortices and high currents, both are responsible for the second band reach the normal state and consequently for this fields are present the phases $I V$ and $V$. The non-composite vortices state are bounded by the phase $I V$. 

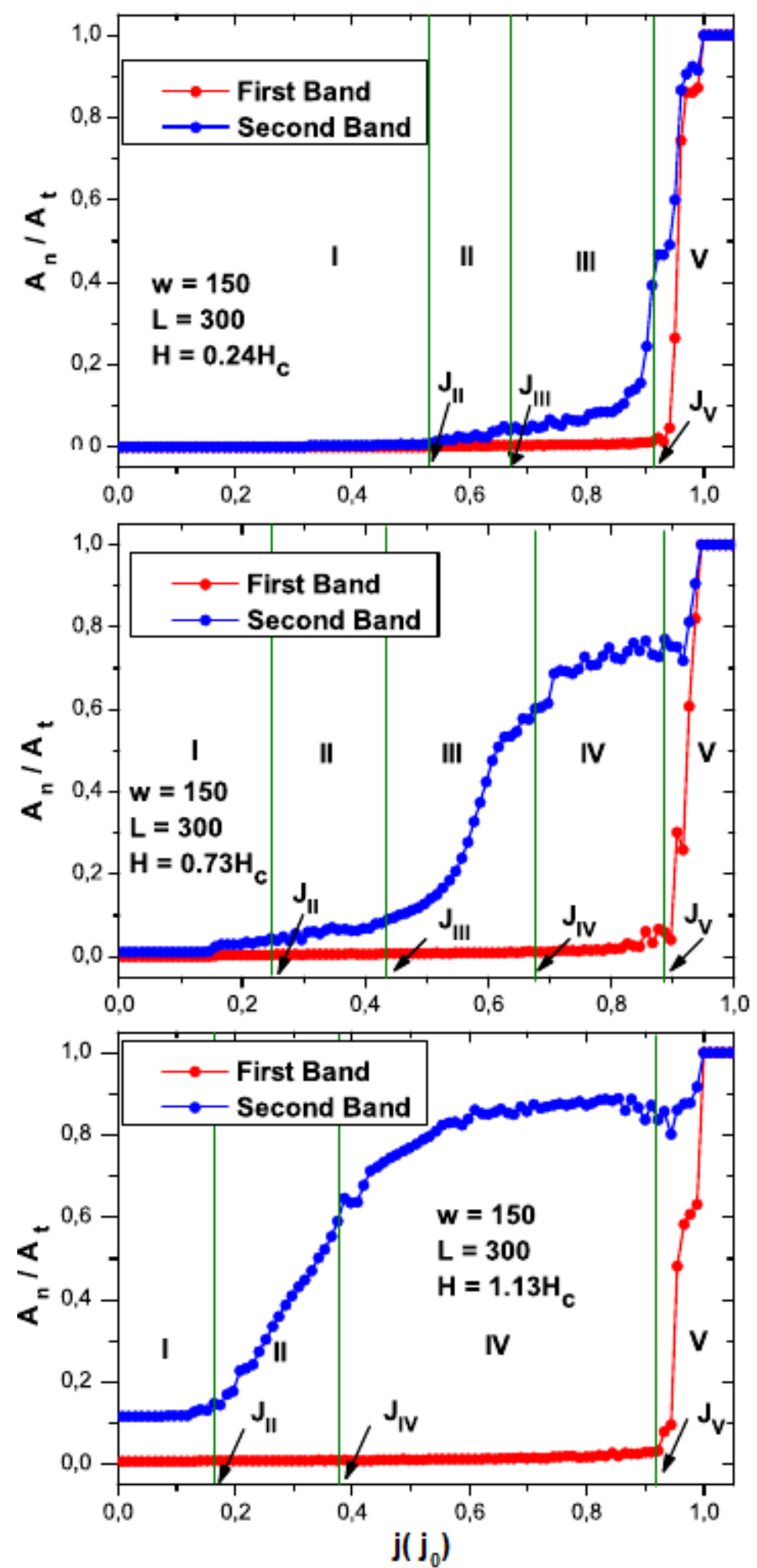

Figure 9: Ratio of the normal state to total area as a function of current for a) $H=0.24 H_{c}$ b) $H=0.73 H_{c}$ and c) $H=1.13 H_{c}$.

\subsection{Interband vortex velocities}

It was discuss in the section 4.3 that the fractional vortex lattice in some band tends to move faster. For some value of external applied current density, the attraction between 
vortices in different bands becomes insufficient to balance the difference in the vortex viscosity and driving force for different bands. As a result, fractional vortex lattices in different bands decouple from each other and they move with different velocities. In our results this threshold currents was named $J_{I I I}$. Effectively we found that for currents $J>J_{I I I}$ the composite vortices are dissociated (Figs. 8.d, 8.g, and 8.h), under constraints for the field exposed in the previous section. But we found that the difference between interband velocities is not the only reason for this dissociation.

We calculed the average of vortex velocities in each band for various field. We believe that the interband velocities becomes apreciably different in the lower half of slab. This task was realize by calculating the time $t$ employed by the vortex to travel some length $d$ in the lower half of the slab. The mean velocity is $d / t$. The Figs. 10 and 11 show the results for $H=0.73 H_{c}$ and $H=0.24 H_{c}$ respectively. For $J>J_{I I I}$ the vortex lattices of each band move with different velocities, in agreement with Lin and Bulaevskii [34]. For the parameters we used, $\xi_{2}=2.24 \xi_{1}$ and the in the first and second band carry respectively a magnetic flux $\Phi_{1}=0.28 \Phi_{0}$ and $\Phi_{1}=0.72 \Phi_{0}$, so the vortices in the second band move faster as expected.

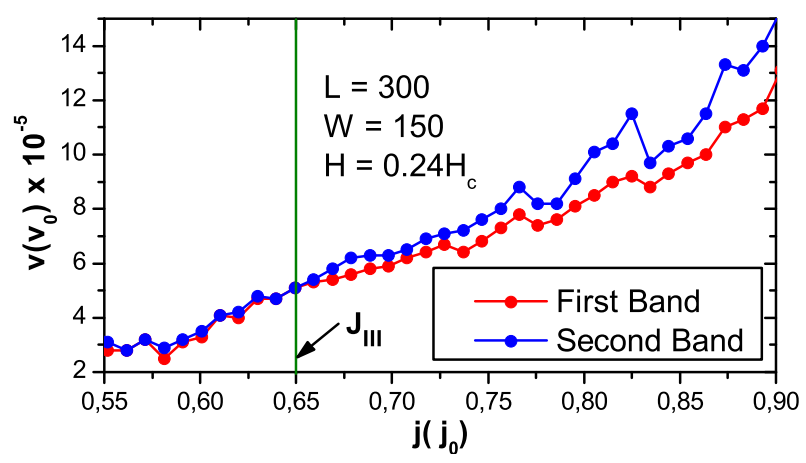

Figure 10: The dependence of velocities of first (red) and second (blue) bands on the current density $j$ for $H=0.24 H_{c}$. These are the average velocities in the lower half of the slab.

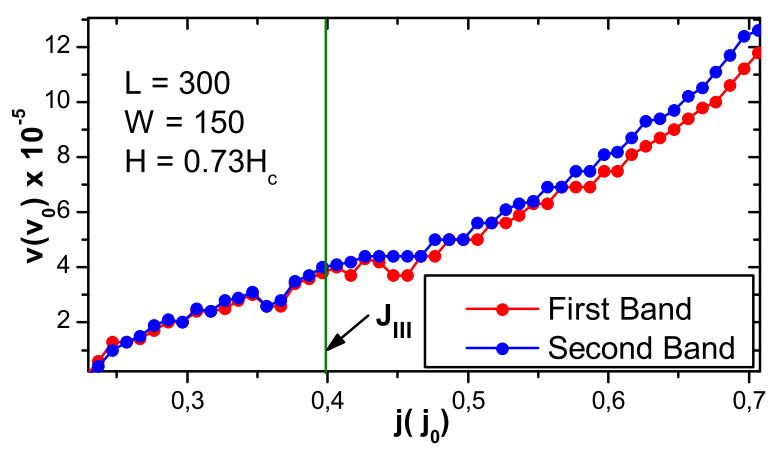

Figure 11: The dependence of velocities of first (red) and second (blue) bands on the current $j$ for $H=0.73 H_{c}$. These are the average velocities in the lower half of the slab.

We present now results for the average vortex velocities performed at the upper half 
of the slab, for $H=0,73 H_{c}$ (Fig. 12). We can see that the velocities are approximately equal in the upper half of slab, as we expected, because the length is not longer enough for the velocities becomes appreciable different.

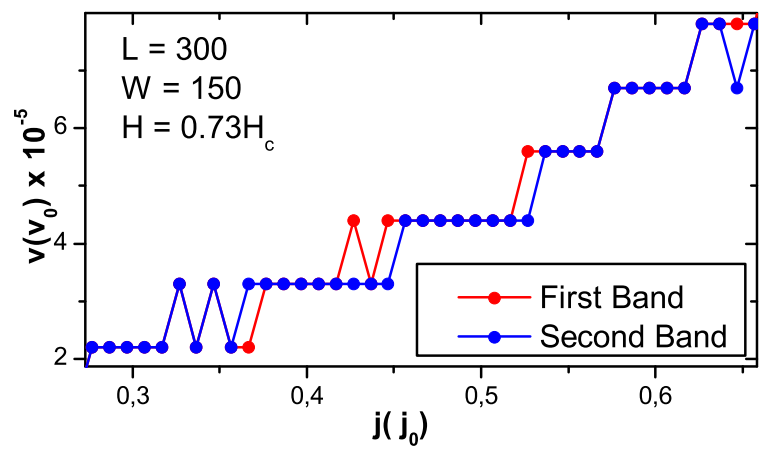

Figure 12: The dependence of velocities of first (red) and second (blue) bands on the current $j$ for $H=0.73 H_{c}$. These are the average velocities in the upper half of the slab.

It is seen in Figs. 8.g - 8.h that for $H=0.73$ the vortices are dissociated in whole slab, then there are other reasons for the dissociation of vortices besides the difference between interband velocities. We believe that the entry rate of vortices and the presence of fractional vortices in the second band at the edge of the slab is other reason. We will be discuss this in the section 4.6 .

\subsection{Correlation as a measure to identify the non-composite vortex state}

For identification of non-composite vortex state, we employed the Pearson product-moment correlation coefficient $r$ (briefly correlation $\mathrm{r}$ ) [113]. It shows a linear relationship between two sets of data. The values of $r$ oscillates between -1 to 1 . In terms of the square modulus of the order parameters of the two condensates the correlation $r$ is defined as follow:

$$
r=\frac{\operatorname{cov}\left(\left|\Psi_{1}\right|^{2}\left|\Psi_{2}\right|^{2}\right)}{\sigma_{1} \sigma_{2}}
$$

where "cov" is the covariance and $\sigma_{1,2}$ is the standard deviation of $\left|\Psi_{1,2}\right|^{2}$. For composite vortices $r \rightarrow 1$ and for non-composite vortices $r \rightarrow 0$.

Figure 13 presents the voltage $V(j)$ and $r(j)$ curves for $H=0.73 H_{c} \sim H_{p}$. The correlation was calculated excluding the effect of frontiers in $x$ direction $(y=0, y=W)$. The points $f, g, h, i$ and $j$ of the diagram (Fig. 8.a) have been represented in the Fig. 13.

In the phases $I$ and $I I$ the vortices are composite and the correlation is high. However in phase $I I$ we observe instabilities (point $p^{*}$ in Fig. 13) leading to dissociation of the vortices (see Fig. 14.a) and sudden decrease in the $r(j)$. In the instability point $p^{*}$ the 
vortex lattice have a transition caracterized by a kink in the $I$ - $V$ curve. Just after the instability the majority of vortices are newly composites (see Fig. 14.b).

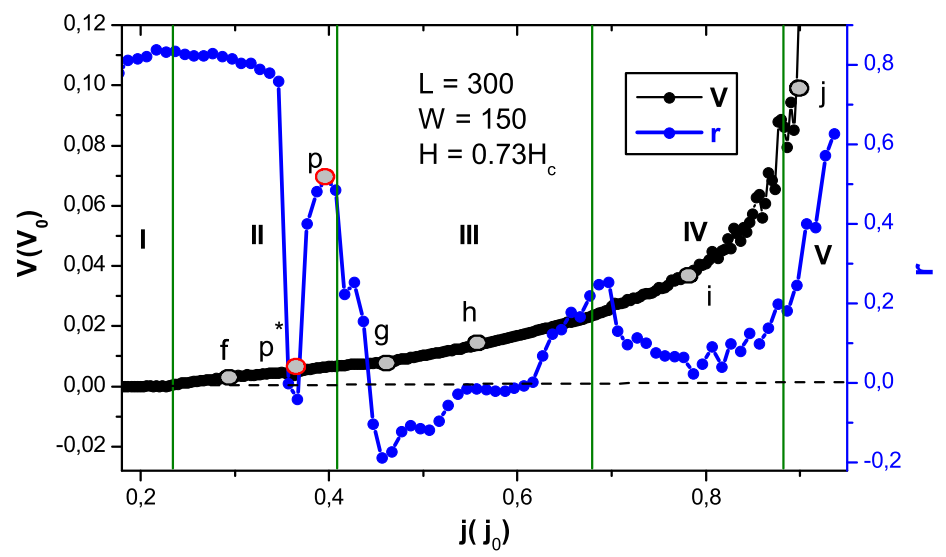

Figure 13: Voltage (black) and correlation (blue) curves in function of current for $H=0,73 H_{c}, W=150$, $L=300$

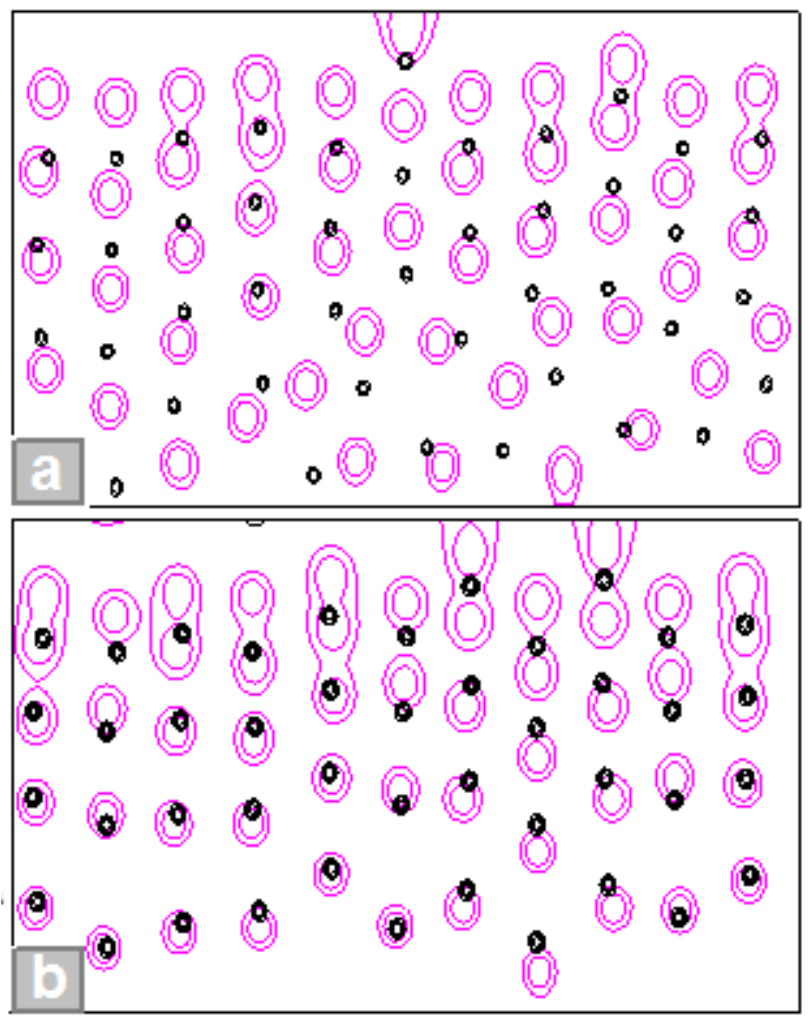

Figure 14: a) Dissociation of vortex lattices in the instability point $p^{*}$ in Fig. 13. b) The vortices reach the composite vortex state newly (point $p$ in Fig. 13) after the instability in $p^{*}$. $W=150, L=300$.

In the phase $I I I$, the correlation start to decrease at $j=J_{I I I}$ and take positive and negative values around the zero, where the vortices are complety dissociated (Figs. 8.g and 8.h). 


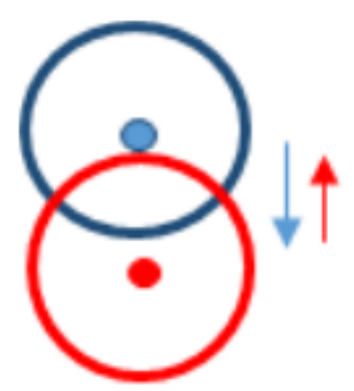

Figure 15: One possible scheme of non-composite vortex. The red vortex is in the first band and the red arrow indicating the direction to increase $\Psi_{1}$. The blue vortex is in the second band and the blue arrow indicating the direction to increase $\Psi_{2}$. Then $r$ is calculated in a direction that $\Psi_{1}$ increase and $\Psi_{2}$ decrease, so $r<0$.

We use Fig. 15 to understand the minus sign obtained for the correlation $\mathrm{r}$. The red vortex is in the first band and the red arrow indicates the direction of increasing $\Psi_{1}$. The blue vortex is in the second band and the blue arrow indicates the direction of increasing $\Psi_{2}$. Then $r$ is calculated in a direction that $\Psi_{1}$ increase and $\Psi_{2}$ decrease, so $r<0$.

In phase $I V$ the first band is responsable for the superconductivity because the majority of the second band is in the normal state (Fig. 8.h). The value of correlation close to zero in this phase does not mean dissociation of vortices.

In phase $V$ the two bands are reaching the normal state (Fig. 8.i). The correlation $r$ is calculated between $\Psi_{1} \rightarrow 0$ and $\Psi_{2} \rightarrow 0$. Thus $r(j)$ is increasing and not mean existence of composite vortices.

In Fig. 16 we present the result for the correlation obtained at a lower field $H=$ $0.24 H_{c}$. The points $b, c, d$ and $e$ are the same on the first dotted line in the diagram Fig. 8.a.

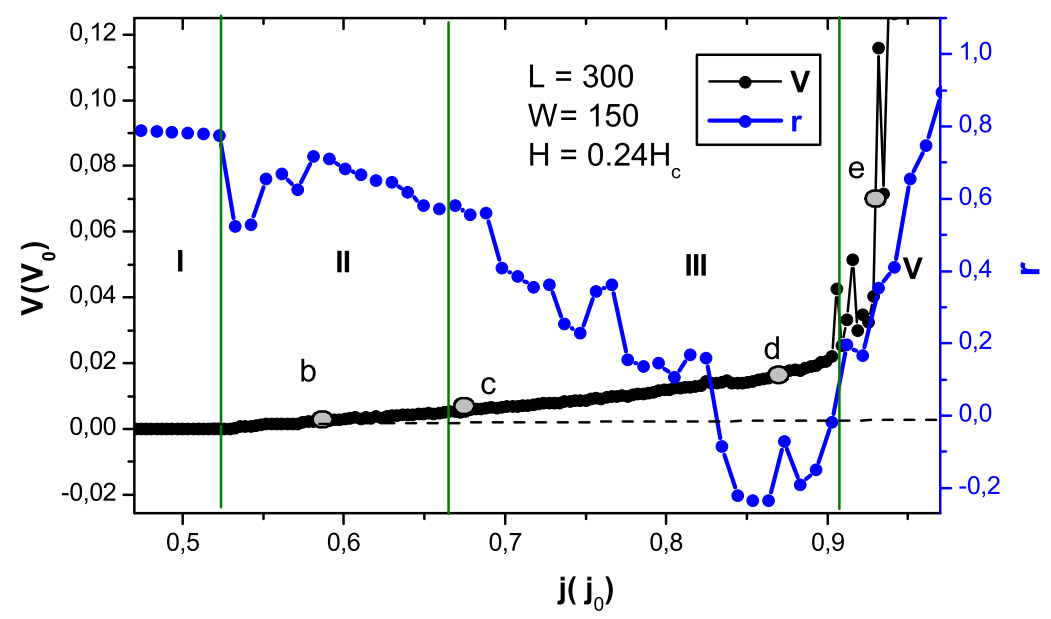

Figure 16: Voltage (black) and correlation (blue) curves for $H=0.24 H_{c}, W=150, L=300$.

We can observe that in the phase $I I I r(j)$ is decrease taking positive and negative values 
as for the higher field. For lower fields the phase $I V$ is absent as was explained in the previous section.

\subsection{Rate of Entrying Vortices}

In order to study qualitatively the influence of the rate (with respect of time) of entrying vortices $(R E V)$ on the dissociation of vortices we will analyse the behavior of the vorticity on the first band $\left(L_{1}\right)$ and correlation as a function of the current density $j$ (Fig. 17). The behavior of $L(j)$ curve is not simple, then we analysed its changes by approximated linear behaviour for particular large and short ranges of currents. Initially, at $j=0$, the vortices have already penetrated the second (weaker) band. However vortices penetrates the strongest band (first band)only at $j \sim 0.147 j_{0}$. The first red line, in Fig.17, reveal that the highest $R E V$ is in the phase $I$, and in this state the vortices are stationary, as discussed in the section 4.3. The first vertical magenta line, in Fig.17, show that in this highest $R E V$ the correlation has a local decrease. Let us see what happen with the vortex lattices in this interval of current. It can be seen in Fig. 17.b that some vortices, pointed by the red arrow, are noticeably deformed. These vortices becoming gradually joined with increasing current (Figs. 17.c, d) until finally performed a complete static composite vortex lattice (Fig. 17.e). It is evidently that once the vortices has penetrated, these need a finite time, namely $\tau_{e q}$, is needed, for reaching at new dynamical configuration of equilibrium. As the vortices penetrated the second band first, the vortex lattice of each band need a different time for reaching the equilibrium $\left(\tau_{e q}^{(1)} \neq \tau_{e q}^{(2)}\right)$. We suppose that this

equilibrium time must to be proportional to the $R E V$, it is $\tau_{e q}^{(1,2)}=\operatorname{const}^{(1,2)} \cdot R E V$. On the other hand, $R E V$ can be defined as $N_{e} / \triangle t$, with $N_{e}$ is the number of vortices that penetrated the sample and $\Delta t$ is the elapsed time for the penetration of the next group of vortices. Then the $R E V$ can be increased by increasing $N_{e}$ or decreasing $\triangle t$. In our simulation $N_{e}$ is changed by increasing the current and consequently $N_{e}$ is high where $L$ is high. For convenience we calculated $L_{1}$ and identified the highest $R E V$ in that points at which the $L_{1}$ increased. The deformed vortices in Figs. 17.b-d. cannot be explained by Lin and Bulaevskii formalism [34], because in the phase $I$ the vortex lattices are static.

The second red line in Fig. 17.a illustrate other local increase in the $R E V$. The correlation have a suddenly decrease and as was show in Fig. 14.a the vortices are suddenly dissociated in $p^{*}$ and further they quickly recombine to form composite vortices (Fig. 14.b).

The orange, yellow and green lines in Fig. 17.a represent three approximated linear behaviour of $L(j)$. We can see that the slope of yellow line at the beginning of the phase $I I I$ is higher than the slope of orange line at the final of phase $I I$. 


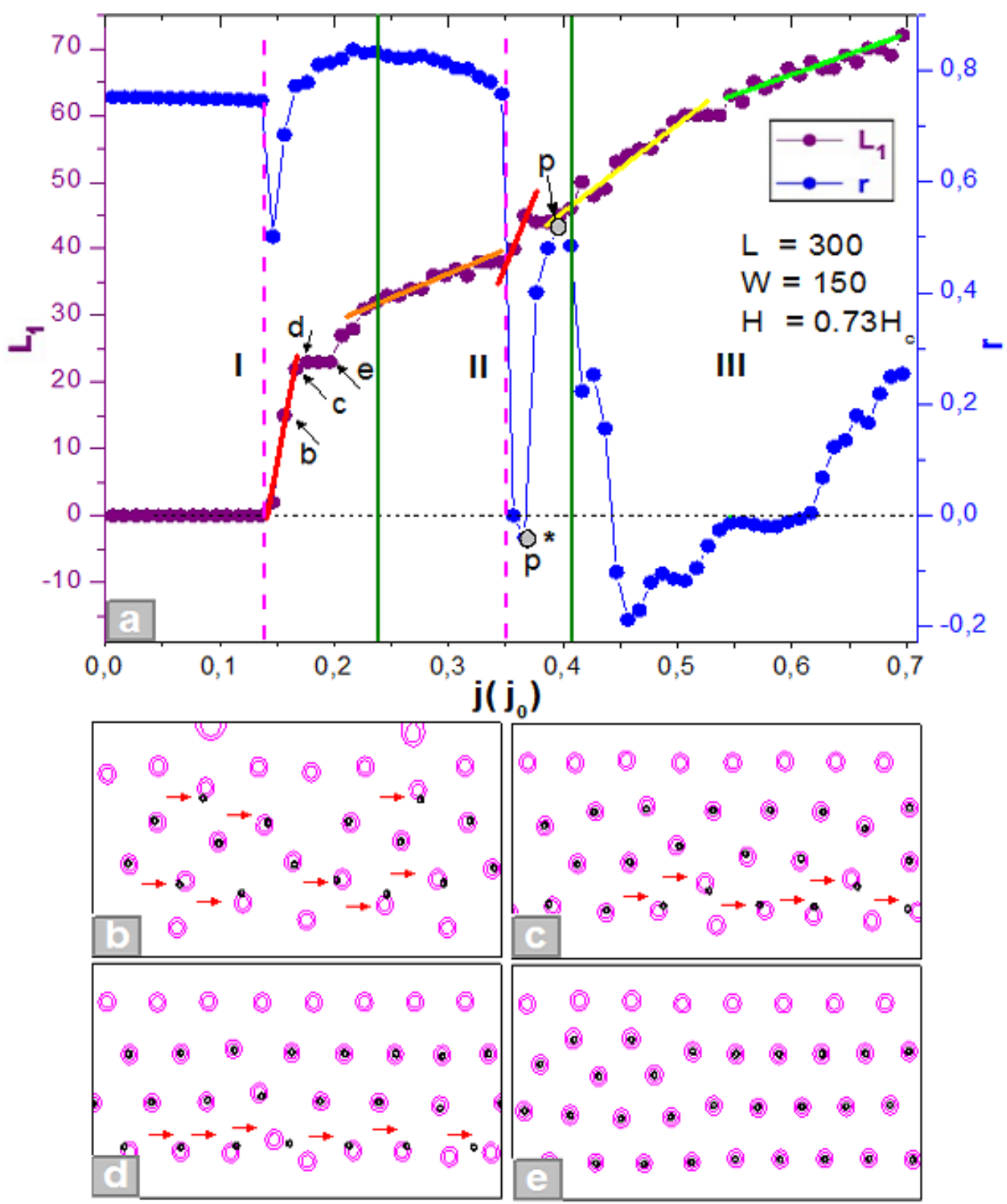

Figure 17: a) Vorticity of the first band $L_{1}$ (violet) and correlation $r$ (blue) in function of current density $j$ for the slab with $L=300, W=150$ and at $H=0.73 \mathrm{Hc}$. The solid green lines separate the phases $I$, $I I$ and $I V$ explain in section 4.3. The vorticity curve (purple) is approximated by several linear behavior of different slopes (red, orange, yellow, and green lines). The magenta vertical lines point at particular sudden decreasing of $r(j)$ curve (blue) accompanied by high local changes (red lines) of vorticity curve (purple) . b)-e) Vortex configuration of states $b, c, d$ and $e$ selected respectively in a). The black (magenta) balls represent the vortices in the first (second) bands. The red arrows point to deformed vortices.

We have seen that the highest $R E V$ favour the dissociation of vortices. This finding complement the prediction of fractional vortex state by Lin and Bulaevskii [34].

For to conclude our analyse of the behavior of $L(j)$ curve we can see that the green line in Fig. 17.a has a smaller slope than the yellow line and that this is manifested in $r(j)$ curve, since $r(j)$ increases at the end of phase $I I I$.

For fields $H<H_{p}$ the results are similar to those already discussed here, but as the 
vorticity is low, the $L(j)$ curve not present appreciable changes.

We can conclude that the dissociation of vortices caused by high $R E V$ is significant only for fields $H \sim H_{p}$. Hence for $H>H_{p}$ the $R E V$ make the second band reach the normal state quickly without dissociation of vortices.

4.7 The influence of Josephson coupling in the dissociation of vortices

The stability regions of fractional state in the $\lambda_{12}-\Phi$ plane ( $\Phi$ is the magnetic flux carrying by vortex)were studied by Piña, et ali., [40]. The cases of $(\mathrm{L}+1, \mathrm{~L})$ were studied. It was found shrinking of the stability regions as $\lambda_{12}$ increases. Guerts, et ali., [38], studied the influence of Josephson coupling $(\gamma)$ in the fractional vortex state and found that by increasing $\gamma$ stabilizes the integer flux states, and destabilizes the fractional states.

In Fig. 18 we show the results of the current density dependence of the correlation, for a slab with $L=150, W=300$ and $H=0.73 H_{c}$, for different strenght values of the Josephson coupling $\lambda_{12}$ (these results are in agreement with those obtained by several authors $[38,40,110])$. We are interested on the behavior of the correlation $(r)$ in the phase $I I I$, which is between the two green dash lines. As we expect, in this phase, $r(\mathbf{j})$ increases with increasing $\lambda_{12}$. From Fig. 18.a we selected some states $b, c, d$ and $e$ (Fig. 18.b-e respectively) and it is revealed that the number of dissociated vortices noticeably decrease when the $\lambda_{12}$ coupling is increased. 


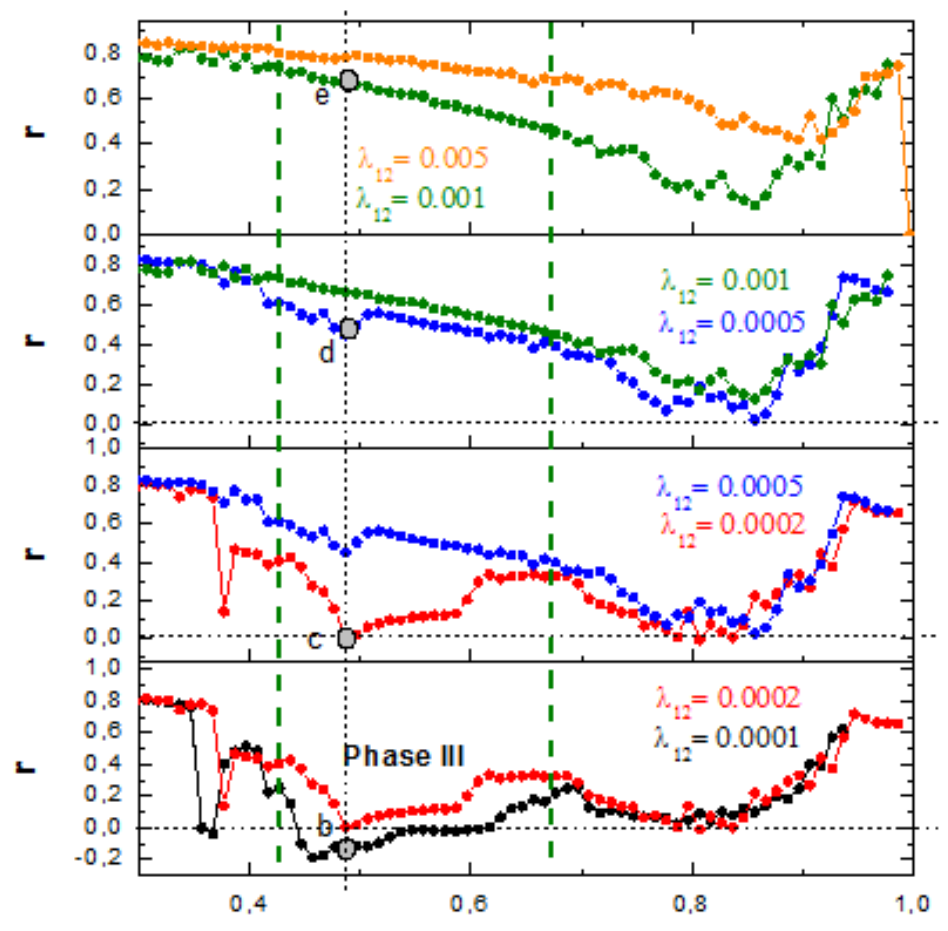

$j\left(j_{N}\right)$
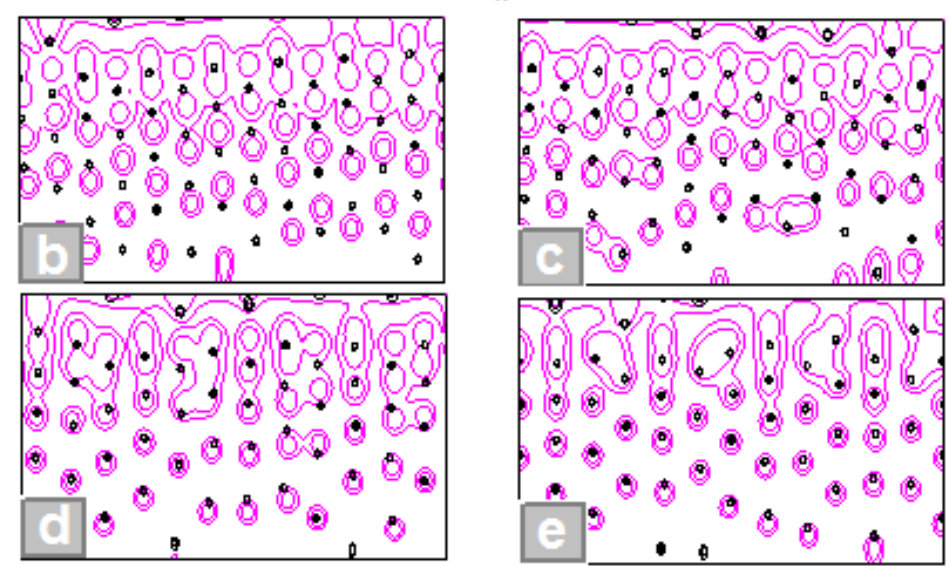

Figure 18: (a) Dependence of the correlation $(r)$ on the current density $(\mathbf{j})$ for different strength of Josephson coupling $\lambda_{12}$ values. $\lambda_{12}=0.0001$ (black curve), $\lambda_{12}=0.0002$ (red), $\lambda_{12}=0.0005$ (blue), $\lambda_{12}=0.001$ (olive), $\lambda_{12}=0.001$ (orange). The phase $I I I$ is between two green dash lines and in this phase the correlation $r(\mathbf{j})$ increase with $\lambda_{12}$ increasing. (b)-(e) Vortex configurations corresponding to selected states $b, c, d$ and $e$, respectively from (a). The black (magenta) balls represent the vortices in the first (second) bands. The dimensions of slab and applied magnetic field are $W=300, L=150$ and $H=0.73 H_{c}$ respectively

The coupling of the superconducting bands effectively reinforce each other and consequently the two band superconductor has a unique $T_{c}$ that is higher than the critical temperatures, $T_{c 1}$ and $T_{c 2}$ of each bands taken separately. We considered the Josephson coupling between the bands in free energy functional (Eq. 2.9.1), resulting from the tunneling of the Cooper pairs from one band to another. This is incorporated trough the term $g_{J}=-\Gamma\left(\Psi_{1}^{*} \Psi_{2}+\Psi_{1} \Psi_{2}^{*}\right)$, where $\Gamma=N(0) \frac{\lambda_{12}}{G}$ and $G=\lambda_{11} \lambda_{22}-\lambda_{12}^{2}$ (see section 2.9 for meaning of other parameters). It is easy to deduce from (Eq. 2.9.1) that the sign of $\Gamma$ determines the relative phase shift between the order parameter in the two condensates, 
zero (0) when $\Gamma>0$ or $\pi$ when $\Gamma<0$ in order for the coupling term to provide a negative energy contribution. However, the sign of $\Gamma$ has no influence on observables such as the Cooper pairs density or the magnetic response of the sample. The general consequence of $\Gamma$ coupling is an injection of Cooper pairs from one band into the other and vice versa, favoring the stability of the superconducting state. Then, the average Cooper-pair density always increases with $\Gamma$.

We can explain our results based in the discussion of Chiboratu et ali., [114]. The Josephson-type interaction favors maximal overlap of order parameters. The free energy functional can be expressed in term of normalized quantities and approximated to the first order in $k^{-2}$

$$
F=\sum_{n} F_{n}\left[\Psi_{n}, \mathbf{A}_{0}\right]-\Gamma \int\left(\Psi_{1}^{*} \Psi_{2}+\Psi_{1} \Psi_{2}^{*}\right)-k^{-2} \iint \frac{j(\mathbf{r}) j\left(\mathbf{r}^{\prime}\right)}{\left|\mathbf{r}-\mathbf{r}^{\prime}\right|}
$$

where $F_{n}\left[\Psi_{n}, \mathbf{A}_{0}\right]$ is the functional of the $n$-th condensate in the unperturbed applied field with the vector potential $\mathbf{A}_{0}$. The last term in Eq. (4.7.1) favors the maximal overlap of the current distributions in the two condensates, it is the maximal similarity of the order parameters. This explains why the phase of the existence of fractional flux vortices shrink with the increase of $\lambda_{12}\left(\Gamma=\right.$ const. $\left.\lambda_{12}\right)$.

\subsection{I-V characteristics}

Lin and Bulaevskii [34], proposed how the dissociation of vortices can be observed experimentally in transport measurements. Taking into account the velocities of the decoupled lattices, the I-V characteristics can be derived from the power balance condition $\eta_{1} v_{1}^{2}+\eta_{2} v_{2}^{2}=J E a^{2}$, where $\eta_{j}, v_{j}$ are the viscosity and velocity of $j$ band, with $j=1,2$. $E$ is the electric field and $a$ is the lattice constant. As can be seen in Fig. 5 there is a notable increase of the differential resistivity $(d E / d J)$ in the decoupled phase.

The decoupling of lattice vortices is not the unique reason for the non-linear increase of superconductivity. Also Larkin-Ovchinnnikov (LO) instability of vortex lattice may be important because the decoupling current is usually large [115]. The vortex core shrinks due to electric field caused by vortex motion and the flux flow resistivity increases with current $[22,23]$.

In Fig 19 we show the $\mathrm{I}-\mathrm{V}$ characteristic for $H=0.73 H_{c}$, for a slab with $\mathrm{L}=300$ and $\mathrm{W}=150$. The red line indicated that the differential resistivity $(d V / d j)$ is higher in phase $I I I$ than in the phase $I I$ (orange line). As we expect, in phase $I I I$ the I-V curve has a non-linear increase of differential resistivity. We believe that, in this phase, the decoupling of lattice and LO instability are the responsible for this non-linear increase, whereas in the phase $I V$ (green line) LO instability is the unique responsible for the non-linear behavior.

The $V(j)$ curve for $H=0.24 H_{c}$ is showed in Fig. 20. As for the previous field studied 


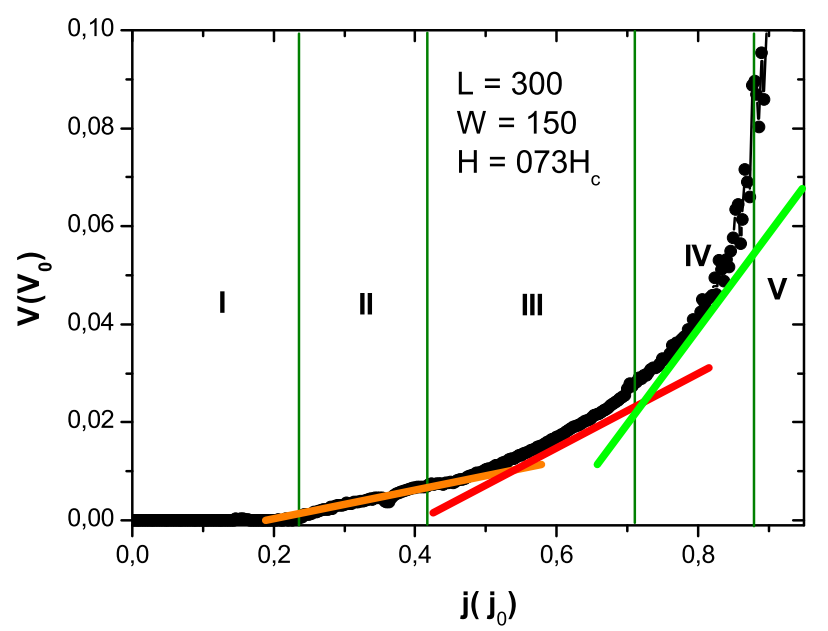

Figure 19: $\mathrm{V}(\mathrm{j})$ curve for $H=0.73 H_{c}, L=300$ and $W=150$. In phase $I I I$ the curve present a non-linear increase due to the decoupling lattice and the LO instability. In phase $I V$ LO instability is the unique responsible for the non-linear increase.

$\left(H=0.73 H_{c}\right)$ field, the red line indicated that the differential resistivity $(d V / d j)$ has a higher change in phase $I I I$ than in the phase $I I$ (orange line). Because of low vorticity and high values of currents in the phase $I I I$, the LO instability has a strong influence in the non-linear increase of $V(j)$ and the decoupled lattice has a small contributions. The strong influence of LO the instability make more noticeable the non-linear increase of resistance at the end of flux flow regime.

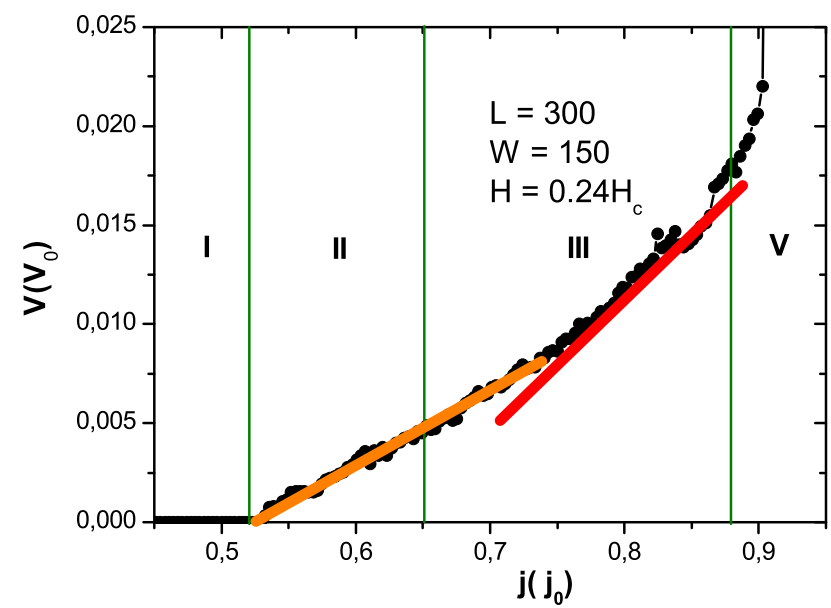

Figure 20: $\mathrm{V}(\mathrm{j})$ curve for $H=0,73 H_{c}, L=300$ and $W=150$. In phase $I I I$ the curve presents a nonlinear behavior due to a strong influence of the LO instability ans small contribution of the decoupling lattice.

We discuss now the $\mathrm{V}(\mathrm{j})$ behavior at a higher $H=1.13 H_{c}$ at which phase $I I I$ not exist. The result are shown in Fig. 21. $\mathrm{V}(\mathrm{j})$ presents a linear behaviour in a large range of flux flow regimen (orange line). As phase $I I I$ is absent the non-linear increase of resistance 
occur at the final of flux flow regimen, where the LO instability is predominant.

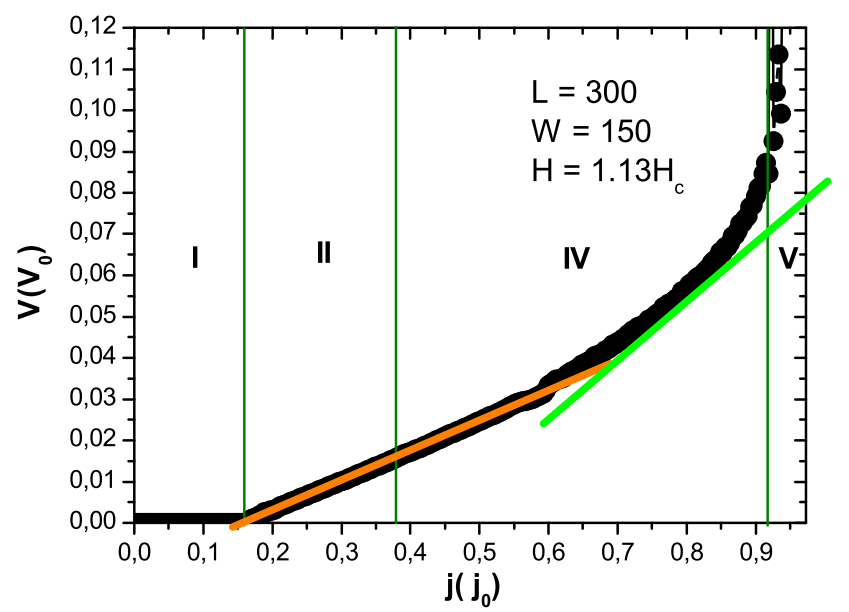

Figure 21: $\mathrm{V}(\mathrm{j})$ curve for $H=1,13 H_{c}, L=300$ and $W=150$.

\subsection{Summary}

Using TCGL formalism we constructed the magnetic field - current density $\mathbf{H}$-j phase diagram that describe the values of magnetic fields and currents for which the composite vortex lattice are dissociated. We found that there are some restriction on the magnetic field for obtaining such dissociated state. The vortex phase are described in reference to the magnetic field at which the vortex penetrate the second (weaker) band in absent the current $\left(H_{p}\right)$.

For $H>H_{p}$ the phase of non-composite vortex (phase III) is absent, since the second band reaches the normal state before the composite vortices are dissociated, whereas the first (strong) band is still active.

The non-composite vortex state (phase $I I I$ ), exist only for $H<H_{p}$ and $H \sim H_{p}$. When $H<H_{p}$ the phase $I I I$ is bounded by a state where that the bands reach the normal state simultaneously, namely phase $V$. For $H \sim H_{p}$ the non-composite vortex state bounded by the phase on which only the first band is active, namely phase $I V$.

High rates of entrying vortices together with the difference between inter-band vortex velocities are responsible for the dissociation of composites vortices. The weaker interband Josephson coupling favour the appearance of non-composite vortex state.

We employed the Pearson correlation coefficient (r) as mathematics tool to identify the non-composite vortices state, taking into account that for composite vortices $r \rightarrow 1$ and for non-composite vortices $r \rightarrow 0$.

Finally the dissociation of vortices can be identified as an increase in the differential resistivity, and also, as a non-linear behavior in the $\mathrm{V}(\mathrm{j})$ curve in the phase III. 


\section{Interband phase textures induced by vortex splitting}

At certain conditions a multiband superconductor can reveal domains of different phase structures with quasi-1D walls between them. Appearance of such domain walls, excited dynamically between regions of contrasting phase difference and decorated by moving vortex sheets, have been predicted for p-wave multi-band superconductors. [1]. However, in two-band s-wave superconductors the phase textures has been predicted theoretically only for quasi-1D structures, where they are induced by imbalances in the normal current through contact interfaces at the sample edges. $[11,116]$

In this chapter we describe another mechanism that induces topological phase textures in a two-band superconductor with s-wave pairing. Contrary to the earlier works $[11,116]$ it produces quasi-1D domains in 2D samples of finite dimensions when a superconductor submited to an dc current is placed in a constant magnetic field. We demonstrate that in such system a non-equilibrium vortex matter is created, forming domains of shifted phases of the band condensates. A key ingredient of this mechanism is the so-called dynamical vortex dissociation, where vortices become fractional. This mechanism does not depend on the special contacts at the ends of the sample and in principle can be found in any multi-band material.

Our analysis is done for a two-band prototype model described by the two-component time-dependent Ginzburg-Landau (TCGL) equations. The difference between the band condensate phases is characterised by the Josephson energy, that enters the free energy functional of the system. A non-equilibrium vortex matter is created by the applied magnetic field in a combination with the flowing current. The non-equilibrium vortex dynamics results in the energy loss, which in turn leads to a finite resistance of the system, reflected in the current-voltage $(I-V)$ characteristics.

\subsection{Introduction}

Appearance of multiple different Cooper pairing channels for different bands in a single superconductor gives rise to a phenomenon of multiband superconductivity, where multiple gaps (condensates) give rise to additional degrees of freedom of the superconducting state. For materials with two bands with the singlet s-wave pairing, such as $\mathrm{MgB}_{2}$, one 
of those degrees of freedom is the difference between phases of the band gap condensates. The phase difference manifests itself, in particular, in the sign of the linear (Josephson) coupling between the bands, which determines the interaction between the band condensates. The degree of freedom related to the phase difference can be excited (driven out of equilibrium) by an external force. Such excitations in systems with many condensates are commonly referred to as the Legget modes [10]. Theoretical studies of their properties have intensified after discovering the multiband superconductivity in materials such as $\mathrm{MgB}_{2}$, [117] iron pnictides [118,119] and others [120]. Experimental evidences of the Legget modes has been published recently [121].

Non-equilibrium configurations of the phase difference between the gap functions can be remarkably nontrivial. For example, in superconductors with three or more bands one has a possibility to achieve a ground state with a broken time-reversal symmetry (BTRS) [122]. Such states are characterised by fixed differences between the band condensate phases being at the same time degenerate. In spatially extended samples this degeneracy can lead to unhomogeneous configurations of the condensate, where domains of different degenerate states alternate, being separated by the domain walls $[123,124]$. Theoretical studies of formation, stabilisation and detection of such domain walls have been recently reported [125-127]. In quasi-one dimensional (1D) samples topological solitons or phase textures can appear, $[11,116,128]$ with a $1 \mathrm{D}$ structure of domain walls. These states are non-equilibrium and are induced by imbalanced carriers due to an injected current. To this date such configurations, however, have not been confirmed experimentally.

\subsection{Model and Method}

The system and method is similar to described in the preceding chapter, but now we focus on domain walls present in our two-component superconducting slab.

Nontrivial phase configurations are typically associated with the appearance of elementary topological structures such as vortices, domains walls, etc. Here we are interested in the phase configurations specific to two-band systems, in particular, we investigate a phase difference between the band condensates $\Delta \theta=\theta_{1}-\theta_{2}$. A natural parameter that characterises such two-band phase differences is the Josephson energy $g_{J}=-2 \gamma\left|\Psi_{1}\right|\left|\Psi_{2}\right| \cos (\theta)$, which describes the inter-condensate interaction and was also used to investigate topological solitons of alternating phases in quasi-1D samples of two-band superconductors. [116]

We also note, that the phase dynamics is necessarily associated with the energy losses, which lead to the finite resistivity of the system. In our case this will be detected from the appearance of a finite potential or voltage across the sample in $x$-direction for a given value of the current density $j$ (the $I-V$ curve). 


\subsection{Vortices near sample boundaries: general picture}

Before presenting results of the numerical calculations we recall that unlike quasi-1D systems, where domain walls (solitons) form non-trivial phase structures, in 2D infinite samples vortices are such elementary building blocks that construct non-trivial topological structures. As mentioned above in infinite equilibrium two-band systems only composite vortices can exist in the central region of slab in the ground state, where vortices in each band share the same centre point and have the same winding number. In the edges of this system fractional vortices with winding number $n_{2}=1$ and $n_{1}=0$, can exist [35]. This is a manifestation of the attraction between vortices in different bands, that is referred for simplicity to as the interband attraction. A signature of this attraction is a negative sign of the Josephson energy for composite vortices. It is easy to see that for fractional vortices the spatial distribution of the Josephson energy has a domain of positive $g_{J}$.

It was explained in the preceding chapter, that vortices enter first in the weaker band and further when the external field is increased the strongest band is penetrated. The difference in the penetration time leads to dynamical separation or "dissociation" of composite vortices and thereby to creation of fractional vortices. This in turn leads to the appearance of domains of $g_{J}>0$, located between separated vortices.

For isolated vortices the interaction with the surface barrier is restricted to the layer of width $l \sim \lambda_{j}$ around the boundary. Once fractional vortices move outside this layer, the influence of the barrier diminishes and vortices tend to become composite again (recombine) due to the attractive interaction of the vortices in different bands (interband attraction). However, this changes for a dense vortex matter, where the distance between vortices is $\sim \lambda_{j}$. In this case the vortex matter is strongly influenced by the interaction between vortices, in particular, due to the additional repulsion between vortices in the same band (intraband repulsion). This may lead to appearance of dynamically unstable but long-lived vortex configurations, with non-trivial spatial distribution of the interband phase difference.

\subsection{Vortex dissociation and phase textures: numerical results}

Numerical calculations of the TCGL equations were done for a sample in the form of a slab with a rectangular crossection with dimensions $L=600 \xi_{1}$ and $w=150 \xi_{1}$. The external magnetic field parallel to $z$-axis, is $H=0.73 H_{c}$ and the other parameters are the same of the preceding chapter.

This vortex state remains stationary if the current is sufficiently small. However, when $j>0.23 j_{0}$ (the current is measured in the units of $j_{0}$, which is the critical current of the slab of the chosen geometry) vortices start to move from the upper boundary $y=W$, which is their entry point, to the lower one at $y=0$, the exit point. Furthermore, penetrating vortices are no longer composite and create domains of positive Josephson 


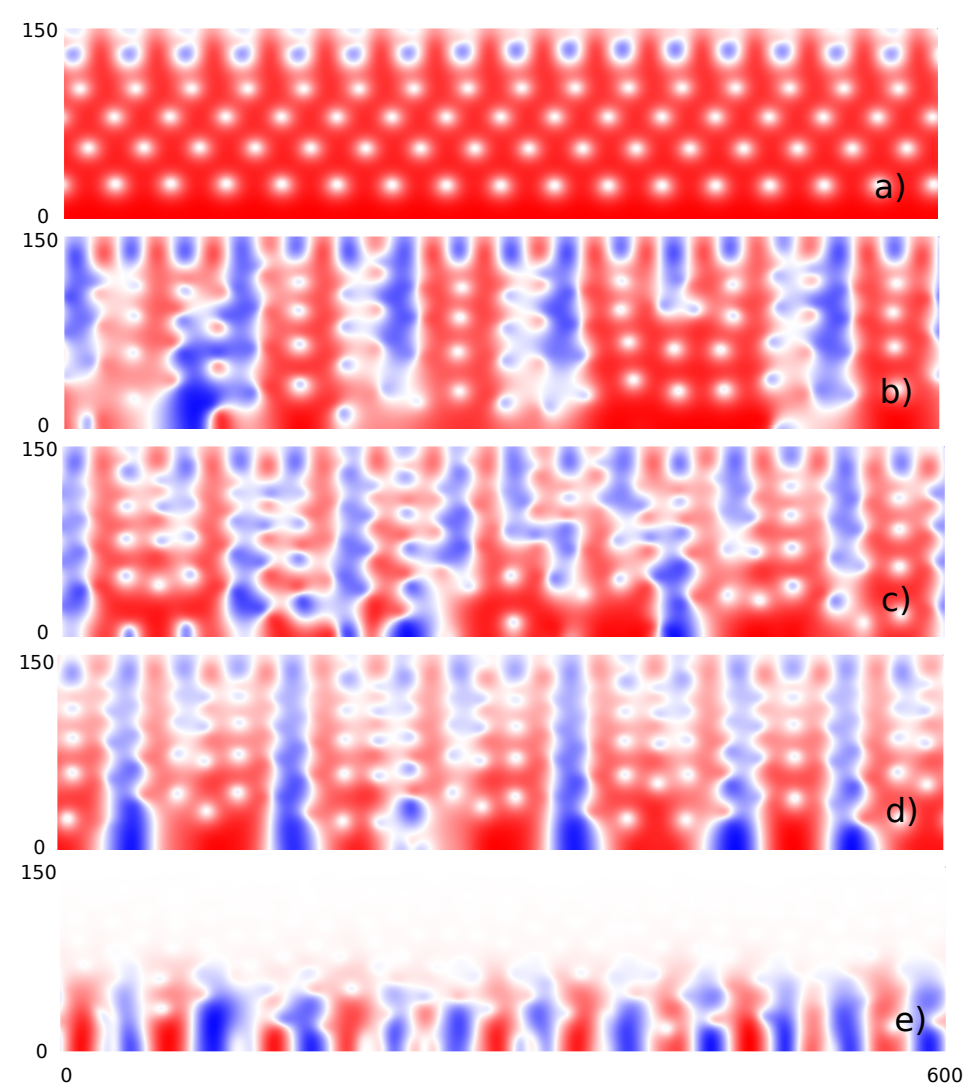

Figure 22: Snapshots of the spatial distribution of the Josephson energy $g_{J}$, plotted as colour density plots, as defined in Eq. (2.9.1), calculated for the slab with the crossection $600 \times 150$ (in the $\xi_{1}$ units). Domains of $g_{J}<0$ and $g_{J}>0$ are marked by red and blue, respectively, colour intensity gives the absolute value $\left|g_{J}\right|$, white colour corresponds to $g_{J}=0$. Panels a), b), c), d) and e) are calculated, respectively, for the current $j=0.33,0.37,0.47,0.57,0.77 j_{0}$. Parameters of the system are discussed in the text.

energy.

This is illustrated in Fig. 22, which shows snapshots of spatial distributions of $g_{J}$, taken at certain time instants, as the colour density plot. Panels a) - e) correspond to few selected values of the applied current $j=0.33,0.37,0.47,0.57,0.77 j_{0}$. Areas with $g_{J}<0$ and $g_{J}>0$ are marked, respectively, red and blue.

Figure 22 a) with $j=0.33 j_{0}$ represents a situation when the current is only slightly above the threshold. Vortices inside the sample are still composite and arranged in the lattice. Still, in the vicinity of the surface fractional vortices appear with small areas of shifted band phases with $g_{J}>0$.

When the current increases fractional vortices start to penetrate deeper inside the sample, forming larger domains of $g_{J}>0$. This is illustrated in Fig. $22 \mathrm{~b}$ ), calculated for $j=0.37 j_{0}$. Domains with $g_{j}>0$ grows with the current. Eventually they reach the lower boundary, as is shown in Fig. $22 \mathrm{c}$ ), calculated for $j=0.47 j_{0}$. When the current further increases, domains of $g_{J}>0$ straighten, forming perpendicular textures [c.f. Fig. $22 \mathrm{~d}$ ) calculated for $j=0.37 j_{0}$ ], similar to solitons observed in quasi-1D systems [116]. For still larger currents the weaker band $j=2$ gradually stops to be superconductive, 
starting from the upper boundary with the largest concentration of vortices One sees this in Fig. $22 \mathrm{e}$ ), where absence of the condensate makes $g_{J}=0$ (white) in the upper part of the sample.

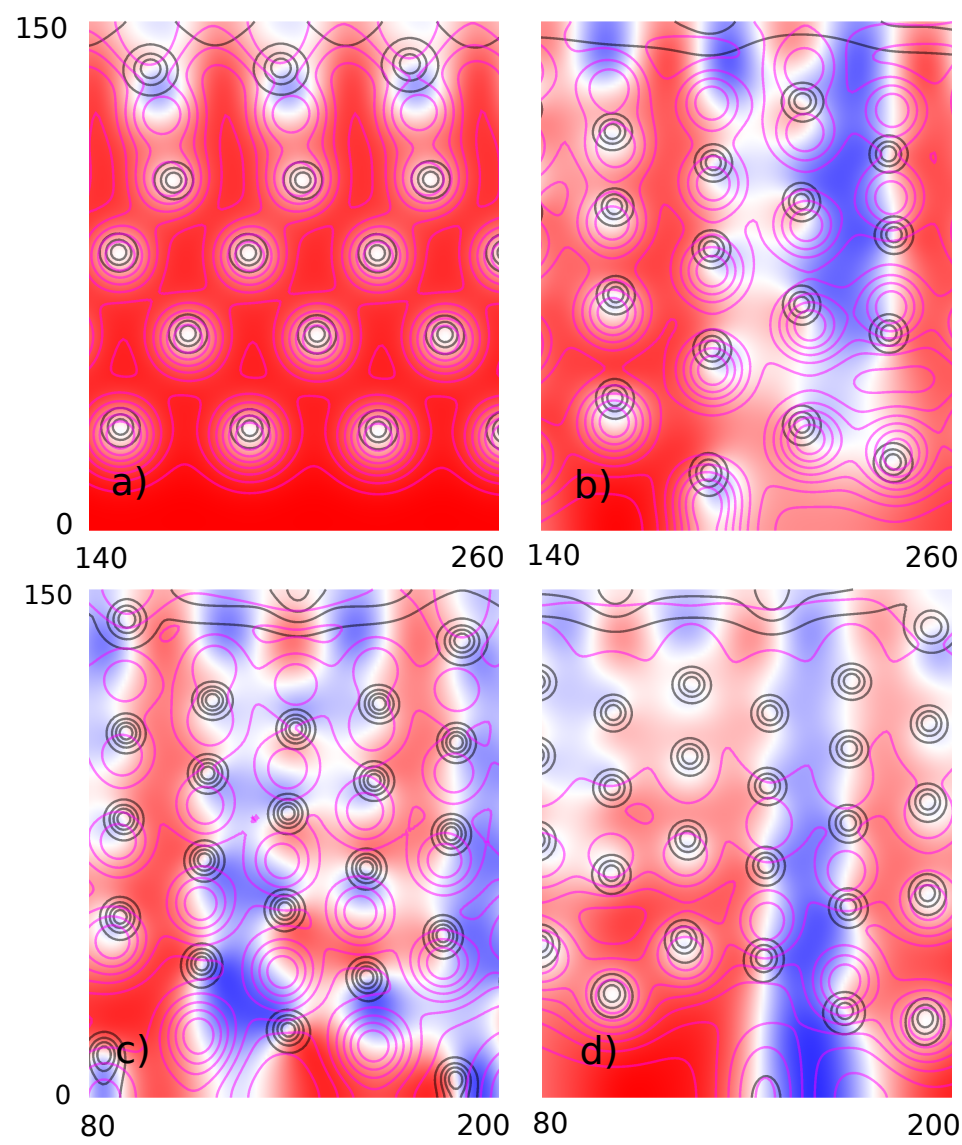

Figure 23: Enlarged parts of the colour density plots of $g_{J}$ shown in Fig. 22, superimposed with the contour plots for the band condensate densities for the strong band $\left|\Psi_{1}\right|$ (black line) and for the weak band $\left|\Psi_{2}\right|$ (magenta line), which reflects the vortex structure in the band condensates.

Further details of the formation of the phase textures and their relation to the process of vortex dissociation are shown in Fig. 23, which plots a scaled part of the $g_{J}$ density plot superimposed with the vortex structure in the bands. The latter are illustrated by the contour lines of the condensate density $\left|\psi_{j}\right|$. Vortices in the stronger band (smaller $\xi_{1}$ ) are represented by the black lines, while those in the weak band (larger $\xi_{2}$ ) are represented by magenta lines. Panels a) - d) in Figs. 22 and 23 are calculated for the same parameters.

One can clearly see, how in the vicinity of the boundary vortices of the weak bands move faster than those in the strong band making vortices fractional. However, those fractional vortices quickly recombine and become composite after leaving the boundary vicinity. For a stronger current the vortex density increased and the recombination is delayed as shown in Fig. $23 \mathrm{~b}$ ). Here, some fractional vortices moving inside the sample arrange themselves in quasi-1D patterns with vortices in one band are located opposite to vortices in the other band. In the middle of such $1 \mathrm{D}$ pattern there are domains of $g_{J}>0$, which stretch towards the lower boundary. Vortices tend to be attracted to this domain 
and to recombine slower. If the current is not so large all vortices recombine when moving deeper inside the sample.

When the current increases further, the recombination is delayed even more and domains of $g_{J}>0$ eventually reach the lower boundary, as in Fig. 23 c) and d). Here the dissociation of vortices that form the domains becomes complete: vortices are separated by distances comparable or exceeding $\xi_{j}$. Finally, Fig. 23 d) also demonstrates how the condensate in the weaker band starts to disappear at the upper boundary, together with the vortex structure.

Figures 22 and 23 demonstrate that quasi-1D domains of the band phase shift is the result of the process where vortices dissociate and arrange themselves in an alternating order, somewhat similar to antiferromagnetic spin ordering. This ordering is related to the balance between the vortex-vortex interactions, which is mainly attractive between vortices in different bands and is repulsive for vortices within the same band. The balance of the interactions depends on the band condensate densities and, therefore, on the current. When the current grows, the condensate is depleted and the repulsion, which is determined by the magnetic field distribution, gradually becomes dominant.

\subsection{Dynamics of the phase textures}

At the initial stationary regimen there are fractional vortices near the edges of the slab and consequently exist a static arrangements of phase textures near the boundaries. Once the flux flow regime started the phase textures move too. Initially, when the phase textures begin to spread into the sample near $j=0.37$ their dynamics is mainly concentrated in the lower part of the sample for the larger phase texture domains. Small phase textures domains travelling from one edge to the other are also present. The Fig 24 illustrates such changes for four different times and constant current $j=0.37 j_{0}$.

When the phase textures channels establish from one edge to another (approximately at $j=0.47$ ), this picture changes somewhat. Near the channels region the behavior is about the same, they are almost static, but between them, there exist some small phase textures regions travel from one edge to the other. This is depicted in Fig. 25.

We can summarize our finding saying that the phase textures are susceptible to changes mainly the larger ones in the lower part of the sample where the vortices exit. This picture holds until the stabilization of the straight channel textures. Since them almost static channels are set. Small phase textures regions travelling from one side to the other are also found in both regimes.

\subsection{Size effects}

As is shown above, the dissociation of vortices which induces interband phase textures is related to the vortex-boundary interactions. One can suppose that this, as well as other 


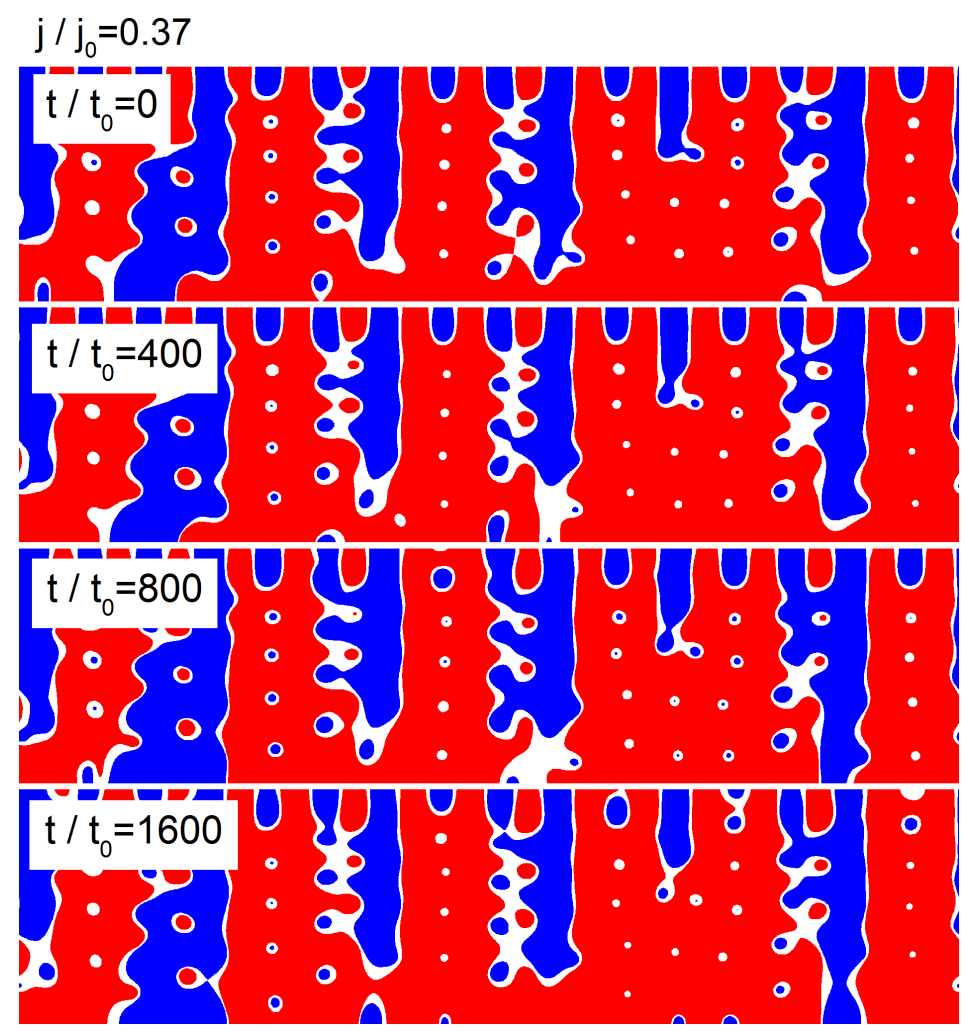

Figure 24: Dynamics of phase textures for a constant current $j=0.37 j_{0}$. The position of phase textures in four different and progressing times, $t=0,400,800$ and $1600 t_{0}$, reveal that this domains move toward the lower boundary $(\mathrm{y}=0)$.

phenomena, related to the dissociation of vortices, strongly depend on the system size. In order to illustrate this dependence we consider dynamics of vortices in slabs of different sizes.

The results are illustrated in Fig. 26, which shows snapshots of the Josephson energy for the slabs with the same $L=600 \xi_{1}$ and two different $W=100 \xi_{1}$, shown in Fig. 26 a), and $W=300 \xi_{1}$, shown in Fig. $26 \mathrm{~b}$ ). Other parameters of the system are the same as in Fig. 22. Figure 26 also shows contour plots for the spatial distribution of the band condensates, $\left|\psi_{j}\right|$, as in Fig. 23.

The value of the current is taken $j=0.447 j_{0}$. For the smaller slab this current corresponds to a situation, where dissociated vortices form perpendicular phase textures with $g_{J}>0$ [c.f. Fig. 26 a)], that already connect the upper and the lower boundaries. However, for the larger slab the vortex distribution and $g_{J}$ change, as shown in Fig. 26 b). Vortices that move towards the lower boundary band gradually recombine so that deep inside the sample only composite vortices are found. This agrees our conclusion that fractional vortices are unstable in a bulk superconductor.

We chose to plot the length of the domain wall, $L_{d w}$, normalized by $w\left(L_{d w} / w\right)$ as function of the external current, $j$. In fig. 27, a set of $L_{d w}(j) / w$ curves are then shown for the sizes $w / \zeta_{1}=100,150$ and 300, in which for each $w$, two values for $H$ are considered, namely, $H / H_{c}=0.61$ and 1.0. These data reveal that the amount of the relative dissocia- 


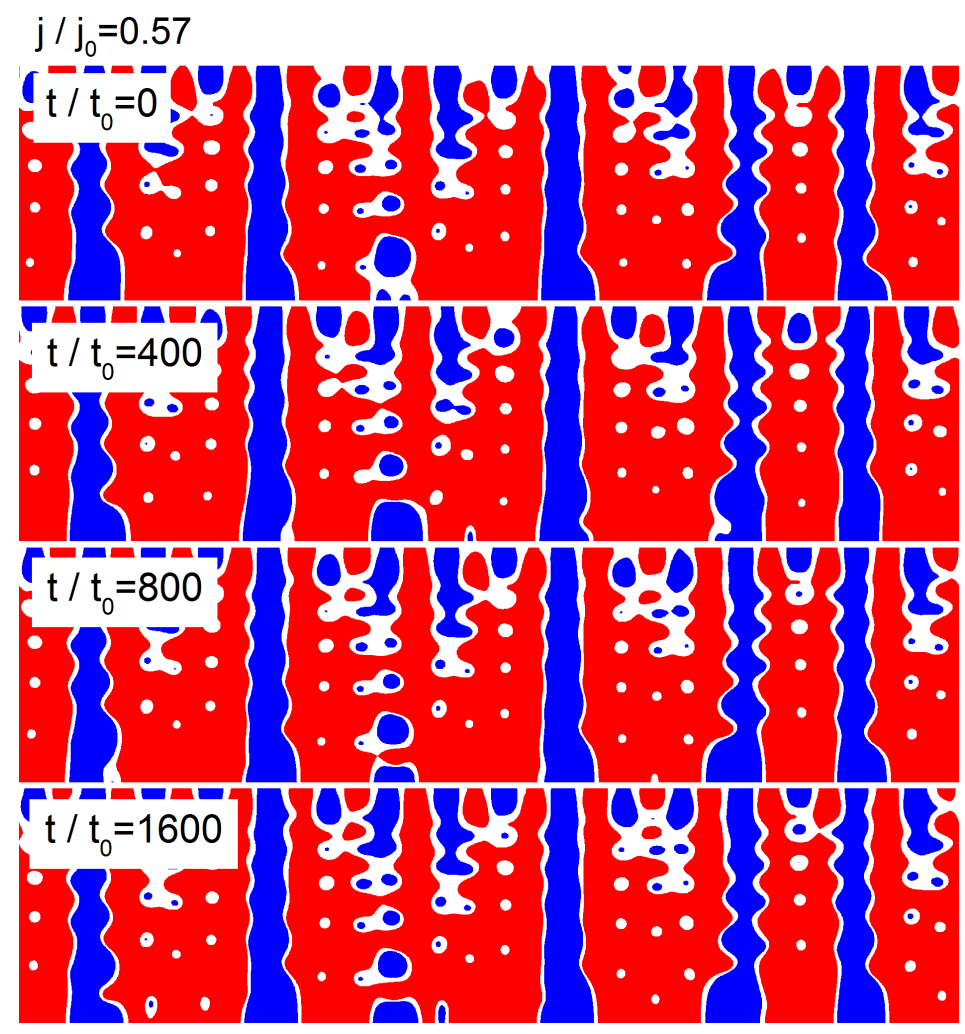

Figure 25: Changes of phase textures for a settled current $j=0.47 j_{0}$. The spacial distribution of phase textures in four different and progressing times, $t=0,400,800$ and $1600 t_{0}$, reveal that the channels are almost static, but between them, some small phase textures regions travel from one edge to the other.

tion (relative because $L_{d w}$ is divided by $w$ ) increases with decreasing $w$ and that all curves present a maximum. It is also shown that for fixed $w$, the larger field $H$ inhibits the size of the domain walls signaling an important dependence of $L_{d w} / w$ with vortex density.

\subsection{Current-voltage (I-V) characteristic}

When vortices start to move their motion gives rise to energy dissipation and to the appearance of a finite voltage between the opposite sample boundaries in the direction of the current. This is illustrated in Fig. 28, which plots the current-voltage (I-V) characteristic of the considered sample for $\lambda_{12}=0,0.0001,0.001$, and 0.005 . While the first three values of the interband coupling correspond to the regime of the phase textures inside the superconductor, the last coupling does not favor vortex splitting inside the sample. Hence, one can check the effect of the phase textures on the dissipation. Notice that the points a - e in Fig. 28 (on the curve for $\lambda_{12}=0.0001$ ) correspond, respectively, to Figs. 22 a) - e) and Figs. 23 a) -d).

From Fig. 28 one sees that the threshold current density (onset of the dissipation) practically does not change in the phase-texture regime. It remains near $0.23 j_{0}$ despite the interband coupling changes by orders of magnitude. However, when the vortex splitting and the related phase textures do not penetrate inside the superconducting sample, the 


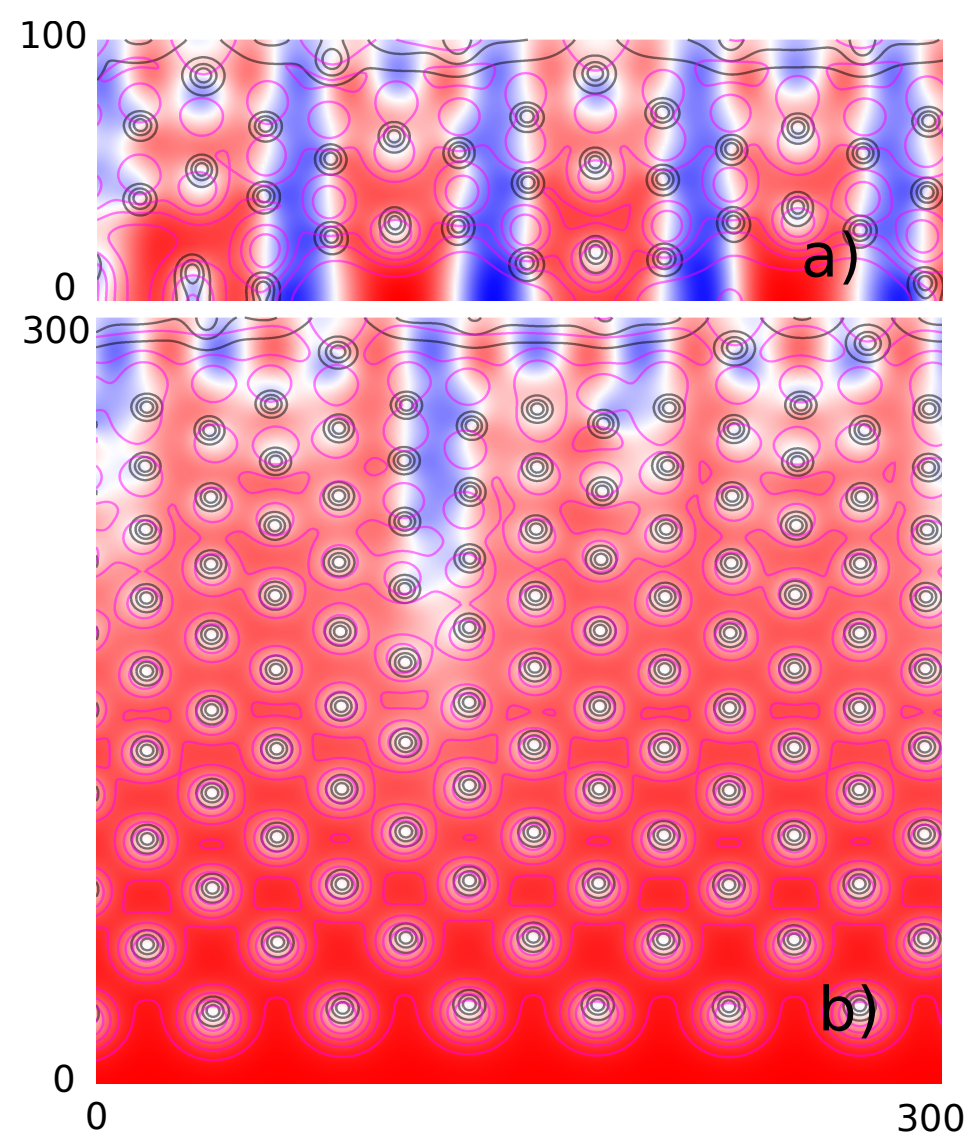

Figure 26: Snapshots of the Josephson energy distributions calculated for the slabs with $L \times w=600 \xi_{1} \times$ $100 \xi_{1}$ (panel a) and with $L \times w=600 \xi_{1} \times 300 \xi_{1}$ (panel b). The contours represent the condensate density $\left|\psi_{1}\right|$ (black lines) and $\left|\psi_{2}\right|$ (magenta lines). The current is $j=0.447 j_{0}$, other parameters are the same as in Fig. 22.

threshold current density shifts down by about $20 \%$, i.e, increasing the impact of the dissipation.

However, strikingly enough, one cannot say generally that the dissipation is less pronounced for the couplings related to the penetration of the phase textures. Indeed, at large current densities the curves for $\lambda_{12}=0,0.0001$ and 0.001 exhibit a higher voltage and higher differential resistance than that of the sample at $\lambda_{12}=0.005$. We arrive at the conclusion that the penetration of the phase textures inside the superconductor has a rather complex effect on the dissipation. For relatively small current densities the dissipation is less significant in the phasetexture regime, with a larger threshold current density for the vortex motion. For large current densities one finds the opposite result, i.e., the dissipation of the energy is more pronounced for the interband couplings favoring the vortex splitting inside the sample.

As also noted above, when the current density is sufficiently large $j>0.707 j_{0}$ the superconductivity in the weak band is destroyed [c.f. Fig. 22 e)]. Then the resistance increases still further [c.f. point e in Fig. 28], because at such strong currents the repulsion between vortices of the remaining condensate weakens and its vortices form a fluid instead of a rigid lattice. In the fluid the mobility of vortices increases considerably, leading to 


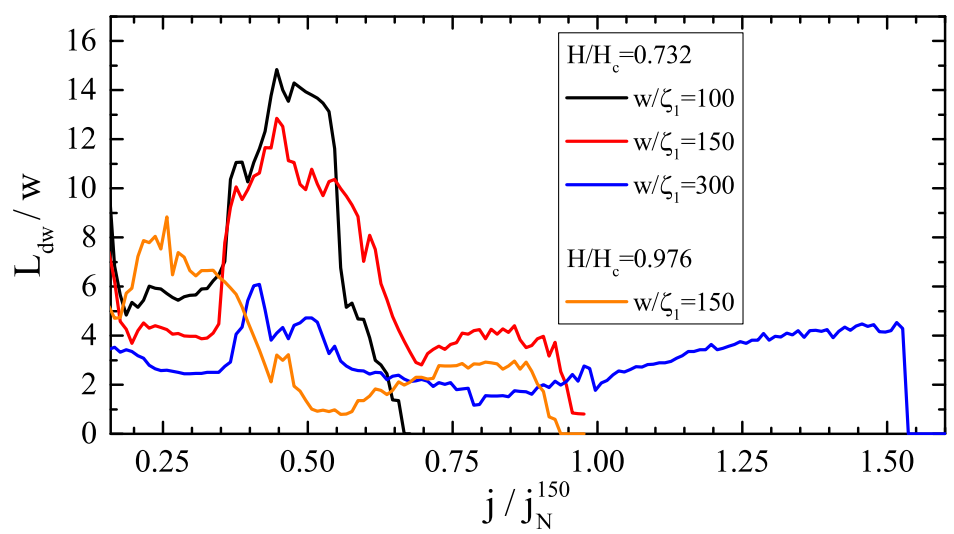

Figure 27: (Color online) Total length of the domain wall normalized by $w, L_{d w} / w$ as function of $j$ for some chosen values of $w$ and $H . L_{d w} / w$ measures the extent of vortex dissociation in the two-band superconducting sample because fractional vortices appear attached to such domain walls.

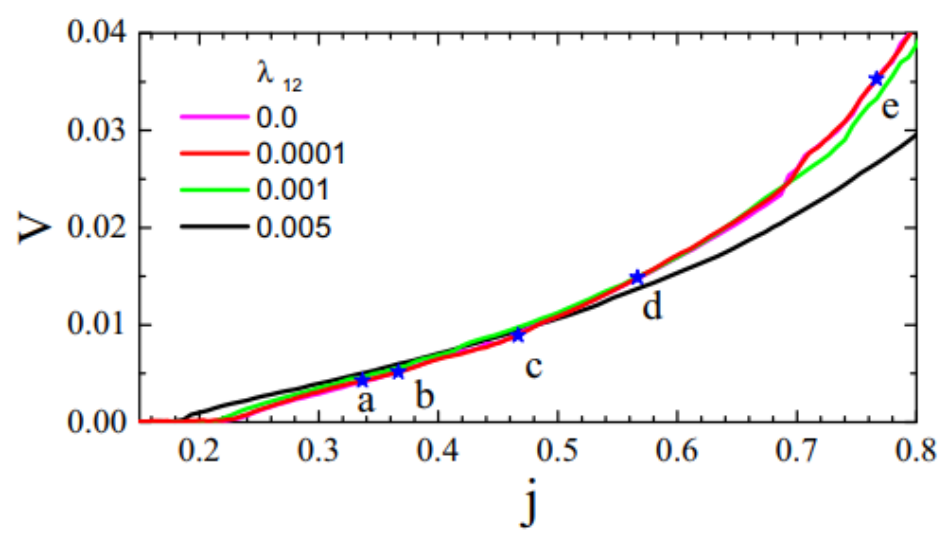

Figure 28: I-V characteristic for the set of the interband coupling $\lambda_{12}=0,0.0001,0.001$, and 0.005 . Points a-e on the curve for $\lambda_{12}=0.0001$ correspond to Fig. 1 a) - e) and Fig. 2 a) - c), respectively. Notice that the I - V characteristic for $\lambda_{12}=0$, and 0.0001 are almost the same.

an increase in the resistance.

\subsection{Summary}

This work investigates the mechanism of the formation of phase textures in a 2D two-band superconductors, placed in the magnetic field. In comparison with equilibrium vortex distribution, the dynamic vortex matter demonstrates a number of specific properties, in particular vortex splitting. Composite vortices split spatially and create fractional vortices, each carrying a non-integer part of the unit quantum flux. The interaction between fractional vortices is a complex pattern, being repulsive for vortices within the same band and attractive for those in different bands. This interaction is responsible for arranging vortices in non-equilibrium alternating patterns, which helps to create quasi-1D phase textures or domains with the changed phase difference between the band condensates. This mechanism for the formation of the interband phase textures, induced by the vortex splitting, differs from that considered previously. We have shown that such phase textures have a significant effect on the dissipation caused by the vortex motion, which deserves 
further investigations. 


\section{Creation of fractional vortices by linear pinning barriers}

We saw that for a two-band superconducting slab with large dimension and free of pinning centers the non-composite vortices are present only in small regions of the sample and for particular values of the applied current. In this chapter we discuss how a different arrangments of linear pinning barriers contribute for the creation of fractional vortices in the whole slab. The linear barriers are sites of the slab at which superconductivity is destroyed. We study the cases where the linear barriers are perpendicular and parallel to the direction of movement of vortices. For the study we first describe a free pinning system with large dimensions and subsenquently we discuss the system with a linear barrier of pinning centers.

\subsection{Introduction}

Artificial pinning center are introduced in a superconducting matrial as a strategy to trap quantum vortices, increasing the maximal magnetic field and applied current that a superconductor can support with minimal dissipation. Several experimental techniques have been employed to produce specifically designed arrays of pinning center have been employed. Among these, pinning centers produced by irradiation with heavy ions [129], chemically grown defects [130], nanostructured perforation [131] and permanent nanomagnets [132]. Domain structure appear in multiband superconductor when some superconducting states are degenerate in free energy. This domains are regions with different energies and between them appear domain wall as topological defects. When a magnetic field is applied some of the vortex are trapped at the domain wall forming an exotic called a vortex sheet [46], where a conventional vortex splits into two vortices with half flux quanta $[12,47,48]$. Machida [1] investigated the flux flow and the pinning of the vortex sheet structure using hexagonal arrays of square pinning center. In the pinning case it is observed an emitting process of a conventional vortex from the vortex sheet by combining a pair of half flux-quantum vortices. 


\subsection{Model and Method}

The system we will discuss in this chapter is exactly the same studied in chapter 4, except that now we introduce via the quantity $\chi_{j}$ in TCGL (Eqs. 2.9.13) spacial function.

For pinning barrier perpendicular to the direction of vortex move, $\chi_{j}$ is defined as follow

$\chi_{j}(x, y)= \begin{cases}\tau-\frac{S_{j}}{n_{j} G}, & \text { out of the region of linear barrier } \\ -\frac{S_{j}}{n_{j} G}, & \text { in the region of linear barrier along } x \text { and with a width } \Delta y\end{cases}$

For pinning barrier parallel to the direction of vortex move, $\chi_{j}$ is defined as follow

$\chi_{j}(x, y)= \begin{cases}\tau-\frac{S_{j}}{n_{j} G}, & \text { out of the region of linear barrier } \\ -\frac{S_{j}}{n_{j} G}, & \text { in the region of linear barrier along } y \text { and width } \Delta x\end{cases}$

The quantities $\tau, S_{j}, n_{j}$ and $G$ were defined in section 2.9 .

We will investigate the behavior of phase difference between the band condensates $\Delta \theta=\theta_{1}-\theta_{2}$, in the same way as was described in section 5.2.

We use the correlation $r$ for describing some characteristic of the free pinning system.

The non-composite vortex state will be detected qualitatively in the pinned system.

6.3 Two-band pinning free superconductor with large dimensions

In the preceding chapter we showed that the process of vortex dissociation induces interband phase textures, it is, domain walls that separate region of slab with positive Josephon energy from the region with negative Josephson energy. 

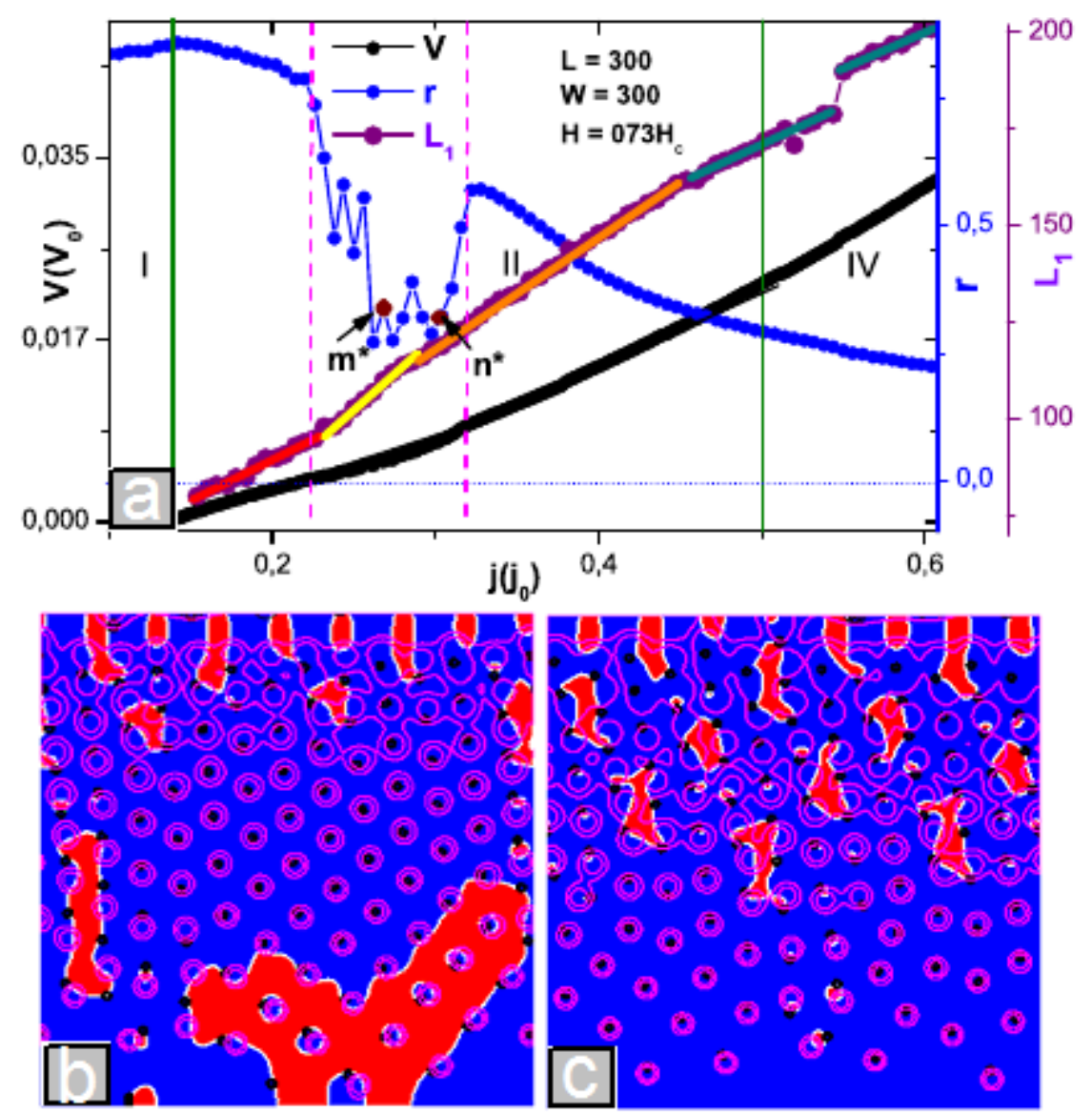

Figure 29: (a) Correlation, voltage and vorticity for the first band (stronger band) curves as a function of the applied current density, for a pinning free two-band superconducting slab, with $H=0.73 H_{c}$, $W=300$ and $L=300$. The solid green lines separate the phases $I, I I$ and $I V$ explain in section 4.3. The behavior of vorticity curve (purple) is approximated by four linear behavior (red, yellow, orange and dark cyan lines). Among this lines, the yellow line has the higher slope, which correspond with a particular decrease of $r(j)$, enclosed between magenta dotted lines (this is agree that we exposed in section 4.6). (b) $m^{*}$ state in $r(j)$ curve from (a): Domains of $g_{J}<0$ and $g_{J}>0$ are marked by red and blue, respectively. White colour corresponds to $g_{J}=0$. Here, $g_{J}$ is the Josephson energy defined in Eq. (2.9.1). The vortices in first (black line) and second (magenta line) bands are plotted. The domain walls do not cross the sample and the vortices are dissociated only in small regions of the sample. In the central region of slab the vortices are composite (c) $n^{*}$ state in $r(j)$ curve from (a): Although the current density of this state is higher than the current density of state $m^{*}$, there is a few dissociated vortices and the normal state in weaker band is more advanced.

It was found that the vortex dissociation process and the formation of domain walls are scarcely present in large dimensions. The non-composite vortices and interband texture only appear at the edges of the slab, but do not cross the sample (see Fig 26). The results presented in Fig.27 show that the length of domain wall decreases as the sample dimension is increased.

In Fig. 29.a we present the correlation, voltage and vorticity for the first band (stronger band) curves as a function of the applied current density. The correlation decrease with the increasing current because the high vorticity and the presence of fractional vortices in the second band, make this weaker band reach the normal state very earlier. This decrease in $r(j)$ curve does not implies in dissocation of composite vortices, as was explain in chapter 
4.

Although the $r(j)$ curve present a particular decreasing region (that enclosed between magenta dotted lines in Fig. 29.a), it is not close to zero and the vortices are only dissociated in specific regions of slab. To illustrate this, in In Fig. 29.b we show the vortex configuration in the minimum point $m^{*}$ of $r(j)\left(j=0.27 j_{0}\right)$. The majority of vortices are composites and small regions close the edges present dissociated vortices.

For the point $n^{*}$ in the same curve for $j=0.3 j_{0}$ the normal state in weaker band is more advanced and few dissociated vortices are observed (Fig. 29.c). The domain walls yet do not cross the slab.

We calculated the length of domain walls as a function of the current density for this dimension and $H=0.73 H_{c}$ (See Fig. 30). The points $m^{*}$ and $n^{*}$ are situated in the maximun of this curve. As we expected the minimum region of $r(j)$ (Fig. 29) correspond with maximum region in the $L(j)$ curve.

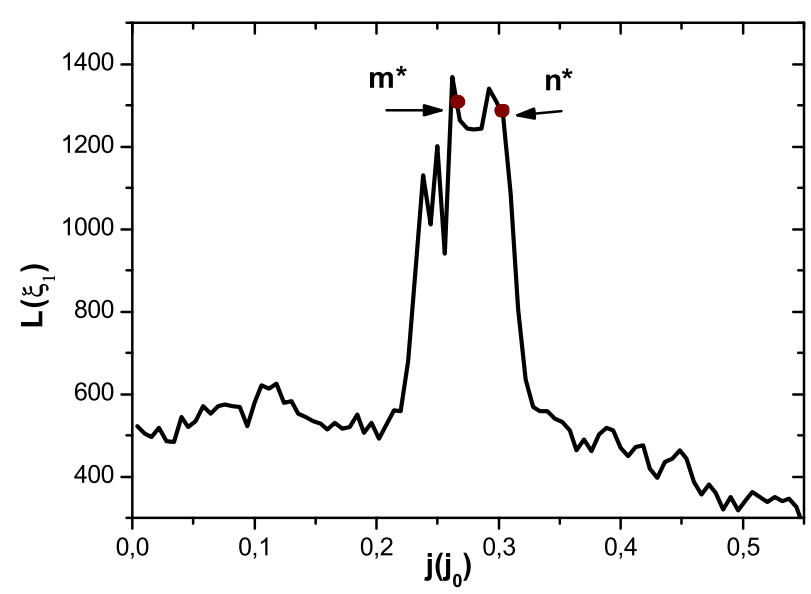

Figure 30: Domain wall length as a function of the applied current density, for a free pinning system, with $H=0.73, W=300$ and $L=300$.

We can see that in the maximum of $L(j)$ curve the domain wall does not cross the sample. Increasing the current does not increase the domain walls length, because the weaker band reaches the normal state, before the vortices dissociation occurs.

On the other hand, in the region where $r(j)$ has a particular decrease, the vorticity presents its highest rate of entrying vortices (yellow line). This is in agreement with what was exposed in section 4.6 .

\subsection{Linear pinning barrier perpendicular to direction of vortex movement}

In this section we used the following criterion for determining the diameter of vortices of each band: We took it as the diameter that cover $80 \%$ of maximum value of the Cooper 
pair density. According to this criterion the vortices diameters in the first and second bands are respectively $D_{1} \approx 6 \xi_{1}$ and $D_{2} \approx 13 \xi_{1}$.

In Fig. 31.a we show the lenght of a the domain wall for a pinning free (red curve) twoband superconducting slab and for the same system with one pinning barrier of width $6 \xi_{1}$, perpendicular to the direction of vortex movement, for $H=0.73 H_{c}$. The slab dimensions are $W=300$ and $L=300$. It is observed a noticeable increase of the domain wall lenght, and, consequently an increase in the number of dissociated vortices, as shown in vortex configuration, Fig. 31.c, of state $m_{1}^{*}$ from Fig. 31.a. 

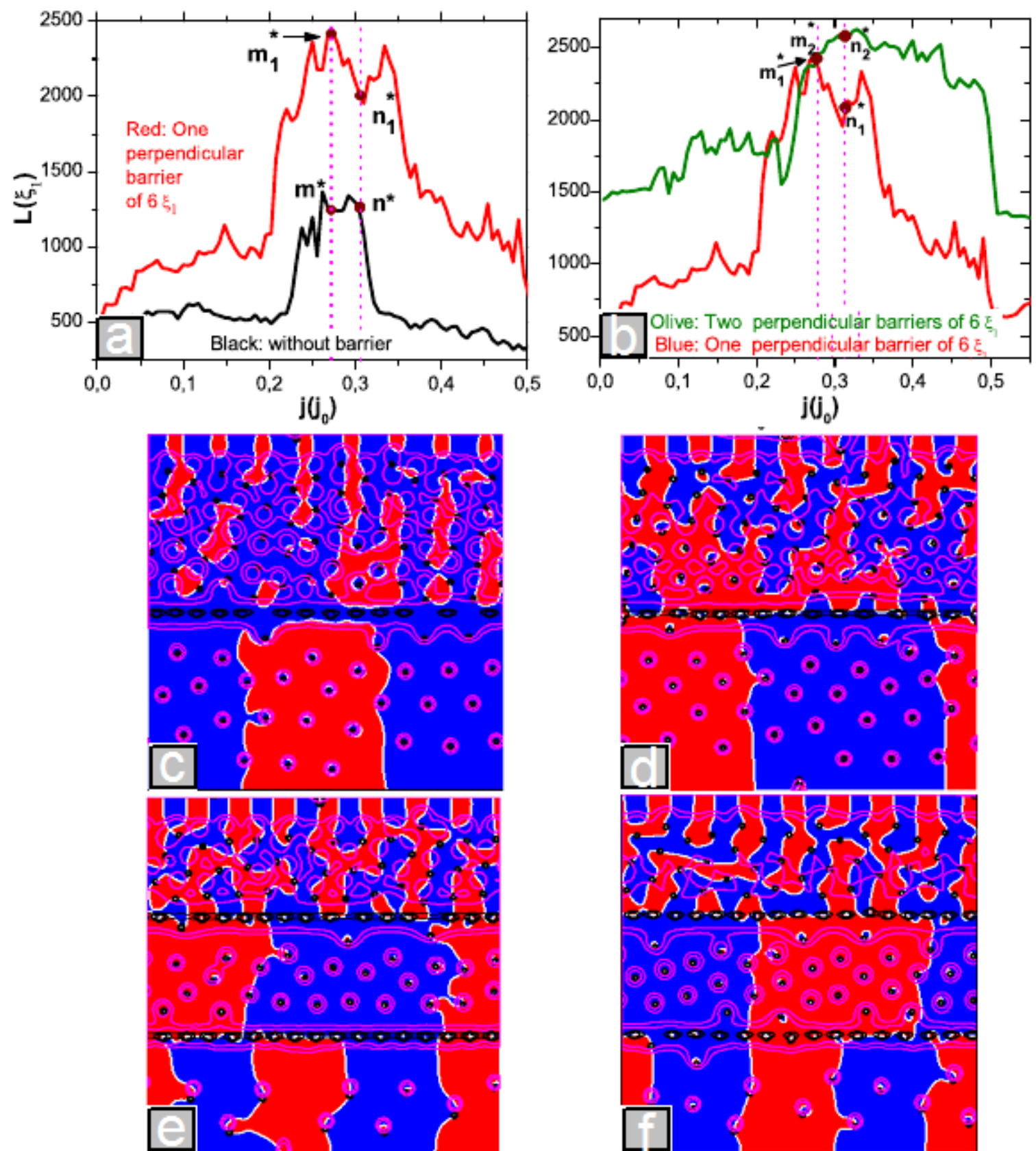

Figure 31: System with $W=300, L=300$ and $H=0.73 H_{c}$. (a) Length of domain walls for a free pinning system (black curve) and for a system with one linear barrier of length $D_{1}$ perpendicular to vortex move (red curve). (b) Length of domain walls for a systems with one (red curve) and two (olive curve) perpendicular linear barrier of length $D_{1}$. From (c) to (f):Domains of $g_{J}<0$ (red) and $g_{J}>0$ (blue). White colour corresponds to $g_{J}=0 . g_{J}$ is the Josephson energy defined in Eq. (2.9.1). The vortices in first (black line) and second (magenta line) bands are plotted. (c) Vortex configuration of state $m_{1}^{*}$ from $\mathrm{L}(\mathrm{j})$ curve in (a). (d) Vortex configuration of state $n_{1}^{*}$ from $\mathrm{L}(\mathrm{j})$ curve in (a). (e) Vortex configuration of state $m_{2}^{*}$ from $\mathrm{L}(\mathrm{j})$ curve in (b). (f) Vortex configuration of state $n_{2}^{*}$ from $\mathrm{L}(\mathrm{j})$ curve in (b).

Fig. 31.c show the vortex configuration for a point $m_{1}^{*}$ with same value current of point $m^{*}$ (Fig. 31.a). We can see that in the superior half of slab the interband vortex cores are dissociated and the external contour of vortices start to overlap, because their high vorticity. 
The inclusion of a linear pinning barrier allow to the domain walls cross the slab. However the vortices are dissociated only in the half superior of slab, since the vortex are dissociated as a consequence of its interaction with the boundaries. The difference in the penetration time leads to a dynamical separation or dissociation of composite vortices and thereby to creation of fractional vortices. When a vortice is trapped at the barrier a new one is emitted. As it does not exist at significant time difference for the emission of vortex in different bands, they are quickly recombined once liberated from the pinning barrier.

Fig. 31.d show the vortex configuration for a point $n_{1}^{*}$ with the same value of current of point $n^{*}$ (see Fig. 31.a). We can see that in the superior half of slab the second band is in advanced normal state and in the lower half the vortices are completely composite.

Introducing two perpendicular pinning barrier, the length of domain walls increase considerably, as we can see in Fig. 31.b. We take two states $m_{2}^{*}$ and $n_{2}^{*}$ in olive curve (system with two perpendicular barrier of length $D_{1}$ ), for the same values of current of points $m^{*}$ and $n^{*}$, respectively. The results are similar as those presented in the preceeding subsection. In the upper part of slab the second band is in normal state, in the middle part the vortices are composite and in the lower part are slightly deformed because they a closer to the lower boundary. Introducing more than one perpendicular barrier does not help the dissociation of vortices since once the vortices are emitted by the pinning barrier they can not interact with the boundary $(y=W)$ that is responsible for the dissociation of vortices.

\subsection{Linear pinning barrier parallel to direction of vortex movement}

Fig. 32.a show the length of domain walls for the same system studied in the previously, with two (olive curve) perpendicular linear barrier of length $D_{1}$ and for two parallel linear barrier of length $2 D_{1}$ (violet curve) as a function of the applied current, for $H=0.73 H_{c}$. The curves have similar behavior.

Now we take the states $m_{3}^{*}$ and $n_{3}^{*}$ in the same values of current of states $m_{2}^{*}$ and $n_{2}^{*}$ (see Fig. 32.a) respectively. We note that the domain walls cross all the slab with the dissociated vortices attached them (Figs. 32.b and 32.c). Some vortices attached to domain walls are composite (Fig. 32.b) because the current is not sufficiently enough for dissociate them. 

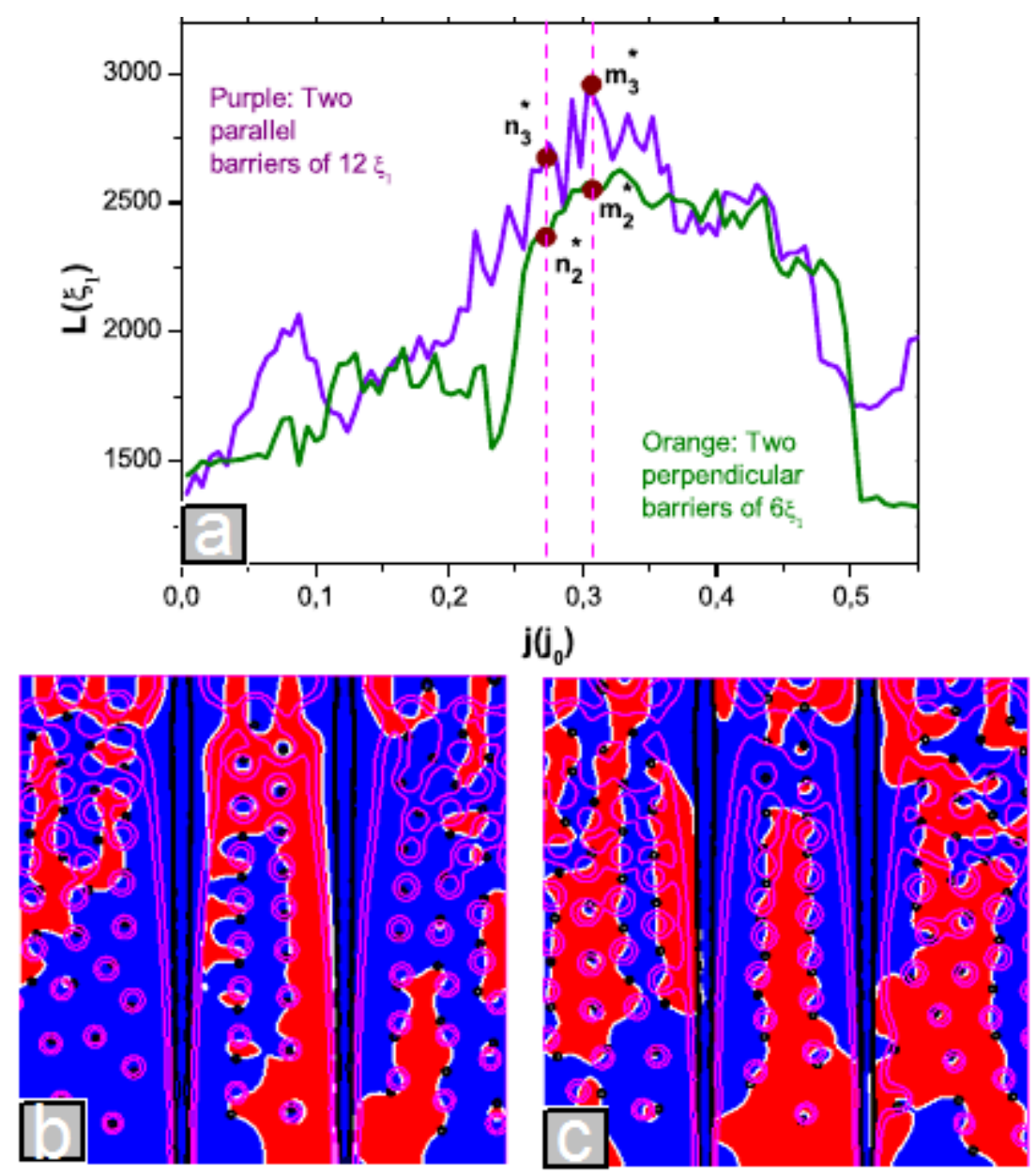

Figure 32: System with $W=300, L=300$ and $H=0.73 H_{c}$ (a)Length of domain walls for a systems with two (olive curve) perpendicular linear barrier of length $D_{1}$ and two parallel linear barrier of length $2 D_{1}$ (violet curve). (b) Domains of $g_{J}<0$ and $g_{J}>0$ are marked by red and blue, respectively. White colour corresponds to $g_{J}=0$. Here, $g_{J}$ is the Josephson energy defined in Eq. (2.9.1). The vortices in first (black line) and second (magenta line) bands are plotted. The domain walls cross the sample with some dissociate vortices attached them. Some vortices attached to domain walls are composite because the current is not sufficiently enough for dissociate them. (c) The domain walls cross the sample with dissociate vortices attached them.

\subsection{Summary}

We have studied the phase texture domain wall and vortex dissociation configurations for a two-band superconducting slab with applied magnetic field and dc current with and without pinning barriers, both perpendicular and parallel to the vortex movement direction. It was shown that for a pinning free slab fractional vortices and domain walls are only present in small regions of the sample. When linear pining barrier are introduced the domain wall cross all the sample (from $y=W$ to $y=0$ ). For pinning barriers perpendicular to the vortex move the vortices are dissociated only in the upper part of slab because the vortices are trapped in the barrier and further newly emitted. Then the interaction with the boundary that is responsible for creation of fractional vortices does not exist anymore. 
For the case of pinning barrier parallel to the vortex move, we get to dissociate the vortices in whole the sample. 


\section{Conclusions}

This work investigates the mechanism of the formation of phase textures in 2D two-band superconductors, placed in the magnetic field. In comparison with equilibrium vortex distribution, the dynamic vortex matter demonstrates a number of specific properties, in particular vortex splitting. Composite vortices split spatially and create fractional vortices, each carrying a noninteger part of the unit quantum flux. The interaction between fractional vortices is a complex pattern, being repulsive for vortices within the same band and attractive for those in different bands. This interaction is responsible for arranging vortices in nonequilibrium alternating patterns, which helps to create quasi-1D phase textures or domains with the changed phase difference between the band condensates. This mechanism for the formation of the interband phase textures, induced by the vortex splitting, differs from that considered previously. We have shown that such phase textures have a significant effect on the dissipation caused by the vortex motion, which deserves further investigation. We have also shown that for superconducting pinning free slab with a large dimension the vortex dissociation procces is delay, but the introduction of linear pinning barrier parallel to the vortex movement facilities the dissociation process. 


\section{References}

[1] Yasushi Matsunaga, Masanori Ichioka, and Kazushige Machida. Flux flow and pinning of the vortex sheet structure in a two-component superconductor. Phys. Rev. B, 70(10):100502, 2004.

[2] ŽL Jelić, M.V. Milošević, Joris Van de Vondel, and A.V. Silhanek. Stroboscopic phenomena in superconductors with dynamic pinning landscape. Scientific Reports, $5,2015$.

[3] J. Bardeen, L. N. Cooper, and J. R. Schrieffer. Theory of superconductivity. Phys. Rev., 108:1175-1204, Dec 1957.

[4] V. A. Moskalenko. Superconductivity of metals with overlapping of energetic bands. FMM, pages 503-513, 1959.

[5] H. Suhl, B. T. Matthias, and L. R. Walker. Bardeen-Cooper-Schrieffer theory of superconductivity in the case of overlapping bands. Phys. Rev. Lett., 3:65, 1959.

[6] T. Muranaka Y. Zenitani J. Akimitsu Nagamatsu J., N. Nakagawa. Superconductivity at $39 \mathrm{k}$ in magnesium diboride. Nature, 410:63, Mar 2001.

[7] S. Raghu, Xiao-Liang Qi, Chao-Xing Liu, D. J. Scalapino, and Shou-Cheng Zhang. Minimal two-band model of the superconducting iron oxypnictides. Phys. Rev. B, 77:220503, Jun 2008.

[8] M. Jourdan, A. Zakharov, M. Foerster, and H. Adrian. Evidence for multiband superconductivity in the heavy fermion compound $U \mathrm{Ni}_{2} A L_{3}$. Phys. Rev. Lett., 93:097001, Aug 2004.

[9] S. V. Shulga, S.-L. Drechsler, G. Fuchs, K.-H. Müller, K. Winzer, M. Heinecke, and K. Krug. Upper critical field peculiarities of superconducting $\mathrm{YNi}_{2} \mathrm{~B}_{2} \mathrm{C}$ and LuNi $i_{2} B_{2}$ C. Phys. Rev. Lett., 80:1730-1733, Feb 1998.

[10] A. J. Leggett. Number-phase fluctuations in two-band superconductors. Progress of Theoretical Physics, 36(5):901, 1966.

[11] Y. Tanaka. Soliton in two-band superconductor. Phys. Rev. Lett., 88:017002, Dec 2001. 
[12] Egor Babaev. Vortices with fractional flux in two-gap superconductors and in extended faddeev model. Phys. Rev. Lett., 89:067001, Jul 2002.

[13] Alexei A Abrikosov. Magnetic properties of superconductors of the second group. Sov. Phys.-JETP (Engl. Transl.);(United States), 5(6), 1957.

[14] Milind N. Kunchur. Unstable flux flow due to heated electrons in superconducting films. Phys. Rev. Lett., 89:137005, Sep 2002.

[15] Milind N. Kunchur, B. I. Ivlev, and J. M. Knight. Steps in the negative-differentialconductivity regime of a superconductor. Phys. Rev. Lett., 87:177001, Oct 2001.

[16] Milind N. Kunchur, Boris I. Ivlev, and James M. Knight. Shear fragmentation of unstable flux flow. Phys. Rev. B, 66:060505, Aug 2002.

[17] A. G. Sivakov, A. M. Glukhov, A. N. Omelyanchouk, Y. Koval, P. Müller, and A. V. Ustinov. Josephson behavior of phase-slip lines in wide superconducting strips. Phys. Rev. Lett., 91:267001, Dec 2003.

[18] John R. Clem, Yasunori Mawatari, G. R. Berdiyorov, and F. M. Peeters. Predicted field-dependent increase of critical currents in asymmetric superconducting nanocircuits. Phys. Rev. B, 85:144511, Apr 2012.

[19] Z. L. Xiao, P. Voss-de Haan, G. Jakob, Th. Kluge, P. Haibach, H. Adrian, and E. Y. Andrei. Flux-flow instability and its anisotropy in $\mathrm{Bi}_{2} \mathrm{Sr}_{2} \mathrm{CaCu}_{2} \mathrm{O}_{8+\delta}$ superconducting films. Phys. Rev. B, 59:1481-1490, Jan 1999.

[20] G. M. Maksimova, N. V. Zhelezina, and I. L. Maksimov. Critical current and negative magnetoresistance of superconducting film with edge barrier. EPL (Europhysics Letters), 53(5):639, Dec 2001.

[21] L. Kramer and R. J. Watts-Tobin. Theory of dissipative current-carrying states in superconducting filaments. Phys. Rev. Lett., 40:1041-1044, Apr 1978.

[22] D. Y. Vodolazov and F. M. Peeters. Rearrangement of the vortex lattice due to instabilities of vortex flow. Phys. Rev. B, 76:014521, Jul 2007.

[23] G. Grimaldi, A. Leo, P. Sabatino, G. Carapella, A. Nigro, S. Pace, V. V. Moshchalkov, and A. V. Silhanek. Speed limit to the abrikosov lattice in mesoscopic superconductors. Phys. Rev. B, 92:024513, Jul 2015.

[24] Laurence Jacobs and Claudio Rebbi. Interaction energy of superconducting vortices. Phys. Rev. B, 19:4486-4494, May 1979. 
[25] Egor Babaev and Martin Speight. Semi-meissner state and neither type-i nor typeii superconductivity in multicomponent superconductors. Phys. Rev. B, 72:180502, Nov 2005.

[26] Shi-Zeng Lin and Xiao Hu. Vortex states and the phase diagram of a multiplecomponent $G$ inzburg-Landau theory with competing repulsive and attractive vortex interactions. Phys. Rev. B, 84:214505, Dec 2011.

[27] Johan Carlström, Julien Garaud, and Egor Babaev. Length scales, collective modes, and type-1.5 regimes in three-band superconductors. Phys. Rev. B, 84(13):134518, Oct 2011.

[28] Egor Babaev, Johan Carlström, and Martin Speight. Type-1.5 superconducting state from an intrinsic proximity effect in two-band superconductors. Phys. Rev. Lett., 105:067003, Aug 2010.

[29] A. Chaves, L. Komendová, M. V. Milošević, J. S. Andrade, G. A. Farias, and F. M. Peeters. Conditions for nonmonotonic vortex interaction in two-band superconductors. Phys. Rev. B, 83:214523, Jun 2011.

[30] E B Bogomolny. The stability of classical solutions. Sov. J. Nucl. Phys., 24(4):449454, 1976.

[31] Akinobu Kanda, BJ Baelus, FM Peeters, Kazuo Kadowaki, and Youiti Ootuka. Experimental evidence for giant vortex states in a mesoscopic superconducting disk. Phys. Rev. Lett., 93(25):257002, Dec 2004.

[32] VA Schweigert, FM Peeters, and P Singha Deo. Vortex phase diagram for mesoscopic superconducting disks. Phys. Rev. Lett., 81(13):2783, Sep 1998.

[33] E. Babaev, A. Sudbø, and N. W. Ashcroft. Observability of a projected new state of matter: A metallic superfluid. Phys. Rev. Lett., 95:105301, Sep 2005.

[34] Shi-Zeng Lin and Lev N. Bulaevskii. Dissociation transition of a composite lattice of magnetic vortices in the flux-flow regime of two-band superconductors. Phys. Rev. Lett., 110:087003, Feb 2013.

[35] M.A. Silaev. Stable fractional flux vortices and unconventional magnetic state in two-component superconductors. Phys. Rev. B, 83(14):144519, Apr 2011.

[36] R.M. da Silva, M.V. Milošević, D. Domínguez, F.M. Peeters, and J. Albino Aguiar. Distinct magnetic signatures of fractional vortex configurations in multiband superconductors. Applied Physics Letters, 105(23):232601, Dec 2014. 
[37] L. F. Chibotaru, V. H. Dao, and A. Ceulemans. Thermodynamically stable noncomposite vortices in mesoscopic two-gap superconductors. EPL (Europhysics Letters), 78(4):47001, May 2007.

[38] R. Geurts, M. V. Milošević, and F. M. Peeters. Vortex matter in mesoscopic twogap superconducting disks: Influence of josephson and magnetic coupling. Phys. Rev. B, 81:214514, Jun 2010.

[39] San Gillis, Juha Jäykkä, and Milorad V. Milošević. Vortex states in mesoscopic three-band superconductors. Phys. Rev. B, 89:024512, Jan 2014.

[40] Juan C. Piña, Clécio C. de Souza Silva, and Milorad V. Milošević. Stability of fractional vortex states in a two-band mesoscopic superconductor. Phys. Rev. B, 86(2):024512, May 2012.

[41] P. E. Cladis, R. D. Parks, and J. M. Daniels. Phase incoherence in the dc superconducting transformer. Phys. Rev. Lett., 21:1521-1524, Nov 1968.

[42] Krishna K. Uprety and Daniel Domínguez. Simple model for the dc flux transformer in layered superconductors with josephson coupling. Phys. Rev. B, 51:5955-5964, Mar 1995.

[43] R. Busch, G. Ries, H. Werthner, G. Kreiselmeyer, and G. Saemann-Ischenko. New aspects of the mixed state from six-terminal measurements on $\mathrm{Bi}_{2} \mathrm{Sr}_{2} \mathrm{CaCu}_{2} \mathrm{O}_{x}$ single crystals. Phys. Rev. Lett., 69:522-525, Jul 1992.

[44] Y. M. Wan, S. E. Hebboul, D. C. Harris, and J. C. Garland. Interlayer Josephson coupling of thermally excited vortices in $\mathrm{Bi}_{2} \mathrm{Sr}_{2} \mathrm{CaCu}_{2} \mathrm{O}_{8-y}$. Phys. Rev. Lett., 71:157-160, Jul 1993.

[45] Shi-Zeng Lin and Charles Reichhardt. Stabilizing fractional vortices in multiband superconductors with periodic pinning arrays. Phys. Rev. B, 87:100508, Mar 2013.

[46] Ü Parts, EV Thuneberg, GE Volovik, JH Koivuniemi, VMH Ruutu, M Heinilä, JM Karimäki, and M Krusius. Vortex sheet in rotating superfluid a 3. Physical review letters, 72(24):3839, 1994.

[47] Manfred Sigrist and Daniel F Agterberg. The role of domain walls on the vortex creep dynamics in unconventional superconductors. Progress of Theoretical Physics, 102(5):965-981, 1999.

[48] Egor Babaev, Ludvig D Faddeev, and Antti J Niemi. Hidden symmetry and knot solitons in a charged two-condensate bose system. Physical Review B, 65(10):100512, 2002. 
[49] A. S. Mosquera Polo, R. M. da Silva, A. Vagov, A. A. Shanenko, C. E. Deluque Toro, and J. Albino Aguiar. Nonequilibrium interband phase textures induced by vortex splitting in two-band superconductors. Phys. Rev. B, 96:054517, Aug 2017.

[50] Fritz London. Une conception nouvelle de la supra-conductibilité: conférences faites à l'Institut Poincaré, Paris. Number 458. Hermann, 1937.

[51] John Bardeen. Two-fluid model of superconductivity. Phys. Rev. Lett., 1:399-400, Dec 1958.

[52] Lev Davidovich Landau and VL Ginzburg. On the theory of superconductivity. Zh. Eksp. Teor. Fiz., 20:1064, May 1950.

[53] M. Tinkham. Introduction to superconductivity. McGraw Hill, New York, 1975.

[54] Pierre Gilles De Gennes. Superconductivity of metals and alloys. Addison-Wesley, 1989.

[55] A.A. Abrikosov. The magnetic properties of superconducting alloys. Journal of Physics and Chemistry of Solids, 2(3):199-208, May 1957.

[56] P.W. Anderson. Theory of flux creep in hard superconductors. Phys. Rev. Lett., $9(7): 309$, Oct 1962.

[57] Philip W. Anderson and Y.B. Kim. Hard superconductivity: theory of the motion of abrikosov flux lines. Reviews of Modern Physics, 36(1):39, Jan 1964.

[58] J. Friedel, P.G. De Gennes, and J. Matricon. Nature of the driving force in flux creep phenomena. Applied Physics Letters, 2(6):119-121, Nov 1963.

[59] Nicole Bontemps, Yvan Bruynseraede, Guy Deutscher, and Aharon Kapitulnik. The Vortex State. Springer, 1994.

[60] E.B. Sonin and B. Horovitz. Thermal and quantum creep of vortices trapped by twin boundaries and columnar defects. Phys. Rev. B, 51(10):6526, Mar 1995.

[61] Ernst Helmut Brandt. Susceptibility of superconductor disks and rings with and without flux creep. Phys. Rev. B, 55(21):14513, Jun 1997.

[62] Ping Ao and David J. Thouless. Berrys phase and the Magnus force for a vortex line in a superconductor. Phys. Rev. Lett., 70(14):2158, Dec 1993.

[63] R.J. Creswick R. Prozorov C.P. Poole. Jr., H.A. Farach. Superconductivity, 2007.

[64] D-X Chen, J.J. Moreno, A. Hernando, A. Sanchez, and B-Z Li. Nature of the driving force on an Abrikosov vortex. Phys. Rev. B, 57(9):5059, Mar 1998. 
[65] C.J. Lobb, David W. Abraham, and M. Tinkham. Theoretical interpretation of resistive transition data from arrays of superconducting weak links. Phys. Rev. B, 27(1):150, Jan 1983.

[66] M.S. Rzchowski, S.P. Benz, M. Tinkham, and C.J. Lobb. Vortex pinning in josephson-junction arrays. Phys. Rev. B, 42(4):2041, Aug 1990.

[67] Jan E. Evetts. Concise encyclopedia of magnetic 83 superconducting materials. Pergamon Press, 1992.

[68] A. Castellanos, R. Wördenweber, G. Ockenfuss, A. vd Hart, and K. Keck. Preparation of regular arrays of antidots in $\mathrm{YBa}_{2} \mathrm{Cu}_{3} \mathrm{O}_{7}$ thin films and observation of vortex lattice matching effects. Applied Physics Letters, 71(7):962-964, May 1997.

[69] V.V. Moshchalkov, M. Baert, V.V. Metlushko, E Rosseel, M.J. Van Bael, Kristiaan Temst, R. Jonckheere, and Yvan Bruynseraede. Magnetization of multiple-quanta vortex lattices. Phys. Rev. B, 54(10):7385, Sep 1996.

[70] L. Van Look, BY Zhu, R. Jonckheere, B.R. Zhao, Z.X. Zhao, and V.V. Moshchalkov. Anisotropic vortex pinning in superconductors with a square array of rectangular submicron holes. Phys. Rev. B, 66(21):214511, Dec 2002.

[71] MJ Van Bael, Kristiaan Temst, VV Moshchalkov, and Yvan Bruynseraede. Magnetic properties of submicron co islands and their use as artificial pinning centers. Physical Review B, 59(22):14674, 1999.

[72] A. Hoffmann, P. Prieto, and Ivan K. Schuller. Periodic vortex pinning with magnetic and nonmagnetic dots: The influence of size. Phys. Rev. B, 61(10):6958, Mar 2000.

[73] Martin Lange, Margriet J. Van Bael, Yvan Bruynseraede, and Victor V. Moshchalkov. Nanoengineered magnetic-field-induced superconductivity. Phys. Rev. Lett., 90(19):197006, May 2003.

[74] O. Daldini, P. Martinoli, J.L. Olsen, and G. Berner. Vortex-line pinning by thickness modulation of superconducting films. Phys. Rev. Lett., 32(5):218, Feb 1974.

[75] A. Hebard, A. Fiory, and S. Somekh. Critical currents in al films with a triangular lattice of $1 \mu \mathrm{m}$ holes. IEEE Transactions on Magnetics, 13(1):589-592, Jan 1977.

[76] A.T. Fiory A.F. Hebard and S. Somekh. Critical currents associated with the interaction of commensurate flux-line sublattices in a perforated ai film. Appl. Phys. Lett, 32:73, Oct 1978. 
[77] V. Metlushko, U. Welp, G.W. Crabtree, R. Osgood, S.D. Bader, L.E. DeLong, Zhao Zhang, S.R.J. Brueck, B. Ilic, K. Chung, et al. Interstitial flux phases in a superconducting niobium film with a square lattice of artificial pinning centers. Phys. Rev. B, 60(18):R12585, Nov 1999.

[78] G.S. Mkrtchyan and V.V. Shmidt. Interaction between a cavity and a vortex in a superconductor of the second kind. Soviet Journal of Experimental and Theoretical Physics, 34:195, Jan 1972.

[79] H. Nordborg and V.M. Vinokur. Interaction between a vortex and a columnar defect in the London approximation. Phys. Rev. B, 62(18):12408, Nov 2000.

[80] I.B. Khalfin and B. Ya Shapiro. Relaxation of magnetic flux in a superconductor with a system of columnar defects. Physica C: Superconductivity, 207(3-4):359-365, Mar 1993.

[81] John Michael Kosterlitz and David James Thouless. Ordering, metastability and phase transitions in two-dimensional systems. Journal of Physics C: Solid State Physics, 6(7):1181, Nov 1973.

[82] Philip Warren Anderson. Basic notions of condensed matter physics. BenjaminCummings, 1984.

[83] Paul M Chaikin and Tom C Lubensky. Principles of condensed matter physics. Cambridge University Press, 2000.

[84] Peter Olsson and Petter Minnhagen. On the helicity modulus, the critical temperature and monte carlo simulations for the two-dimensional XY-model. Physica Scripta, 43(2):203, Dec 1991.

[85] Lawrence Yun Lung Shen, N. M. Senozan, and Norman E. Phillips. Evidence for two energy gaps in high-purity superconducting nb, ta, and v. Phys. Rev. Lett., 14:1025-1027, Jun 1965.

[86] Daniel R. Slocombe, Vladimir L. Kuznetsov, Wojciech Grochala, Robert J. P. Williams, and Peter P. Edwards. Superconductivity in transition metals. Philosophical transactions. Series A, Mathematical, physical, and engineering sciences, 373(2037), March 2015.

[87] L. F. Mattheiss. Band structures of transition-metal-dichalcogenide layer compounds. Phys. Rev. B, 8:3719-3740, Oct 1973.

[88] Y. Koike, H. Suematsu, K. Higuchi, and S. Tanuma. Superconductivity in graphitealkali metal intercalation compounds. Physica B+C, 99(1):503 - 508, 1980. 
[89] N. B. Hannay, T. H. Geballe, B. T. Matthias, K. Andres, P. Schmidt, and D. MacNair. Superconductivity in graphitic compounds. Phys. Rev. Lett., 14:225-226, Feb 1965.

[90] Roger Chevrel, Marcel Sergent, and Jacques Prigent. Sur de nouvelles phases sulfurées ternaires du molybdène. Journal of Solid State Chemistry, 3(4):515 - 519, 1971.

[91] Hideo Ihara, Kazuyasu Tokiwa, Hirotomo Ozawa, Masayuki Hirabayashi, Hirofumi Matuhata, Akira Negishi, and Young Seok Song. New high- $T_{c}$ superconductor $A g_{1-x}$ $\mathrm{Cu}_{x} \mathrm{Ba}_{2} \mathrm{Ca}_{n-1} \mathrm{Cu}_{n} \mathrm{O}_{2 n+3-\delta}$ family with $\mathrm{T}_{c}>117 \mathrm{k}$. Japanese Journal of Applied Physics, 33(3A):L300, 1994.

[92] N. Hamada and H. Ihara. Electronic band structures of $\mathrm{CuBa} \mathrm{Ca}_{\mathrm{n}-1} \mathrm{Cu} \mathrm{n}_{\mathrm{n}} \mathrm{O} 2_{\mathrm{n}+2}$ and $\mathrm{CuBa} a_{2} \mathrm{Ca}_{\mathrm{n}-1} C u_{\mathrm{n}} \mathrm{O}_{2 \mathrm{n}+1} F(n=3-5)$. Physica $C$ : Superconductivity, 357-360(Part 1):108 - 111, 2001.

[93] Y. Tokunaga, K. Ishida, Y. Kitaoka, K. Asayama, K. Tokiwa, A. Iyo, and H. Ihara. Effect of carrier distribution on superconducting characteristics of the multilayered high- $T_{c}$ cuprate $\left(C u_{0.6} C_{0.4}\right) B a_{2} C a_{3} C u_{4} O_{12+y}:{ }^{63} \mathrm{Cu}-$ NMR study. Phys. Rev. B, 61:9707-9710, Apr 2000.

[94] Y. Tanaka, A. Iyo, N. Shirakawa, M. Ariyama, M. Tokumoto, S.I. Ikeda, and H. Ihara. Specific heat study on $\mathrm{Cu}_{x} \mathrm{Ba}_{2} C a_{\mathrm{n}-1} C u_{\mathrm{n}} \mathrm{O}_{y}$. Physica $C$ : Superconductivity, 357-360(Part 1):222 - 225, 2001.

[95] Yoichi Kamihara, Hidenori Hiramatsu, Masahiro Hirano, Ryuto Kawamura, Hiroshi Yanagi, Toshio Kamiya, and Hideo Hosono. Iron-based layered superconductor:LaOFeP. Journal of the American Chemical Society, 128(31):10012-10013, 2006. PMID: 16881620.

[96] J. Peretti. Superconductivity of transition elements. Physics Letters, 2:275-276, October 1962.

[97] Takashi Yanagisawa and K. Hyobanshi. Quarks and fractionally quantized vortices in superconductors: an analogy between two worlds. Recent Advances in Quarks Research, pages 113-46, 2013.

[98] Y.M. Cho and P.M. Zhang. Topological objects in two-gap superconductor. The European Physical Journal B-Condensed Matter and Complex Systems, 65(2):155178, Sep 2008. 
[99] Egor Babaev, Ludvig D Faddeev, and Antti J Niemi. Hidden symmetry and knot solitons in a charged two-condensate bose system. Physical Review B, 65(10):100512, Feb 2002.

[100] Y.M. Cho and Pengming Zhang. Non-abrikosov vortex and topological knot in two-gap superconductors. Physical Review B, 73(18):180506, May 2006.

[101] Minoru Eto, Yuji Hirono, Muneto Nitta, and Shigehiro Yasui. Vortices and other topological solitons in dense quark matter. Progress of Theoretical and Experimental Physics, 2014(1), Jan 2014.

[102] IP Ivanov. General two-order-parameter ginzburg-landau model with quadratic and quartic interactions. Physical Review E, 79(2):021116, Feb 2009.

[103] YS Yerin and AN Omelyanchouk. Coherent current states in a two-band superconductor. Low Temperature Physics, 33(5):401-407, Jun 2007.

[104] AG Shepelev. Gap anisotropy in the energy spectrum of superconductors. PhysicsUspekhi, 11(5):690-711, Dec 1969.

[105] Alan J Bennett. Theory of the anisotropic energy gap in superconducting lead. Physical Review, 140(6A):A1902, Dec 1965.

[106] A Sanna, G Profeta, A Floris, A Marini, EKU Gross, and S Massidda. Anisotropic gap of superconducting cac 6: a first-principles density functional calculation. Physical Review B, 75(2):020511, Dec 2007.

[107] VG Kogan and Joerg Schmalian. Ginzburg-Landau theory of two-band superconductors: Absence of type-1.5 superconductivity. Phys. Rev. B, 83(5):054515, Feb 2011.

[108] William D. Gropp, Hans G. Kaper, Gary K. Leaf, David M. Levine, Mario Palumbo, and Valerii M. Vinokur. Numerical simulation of vortex dynamics in type-II superconductors. Journal of Computational Physics, 123(2):254-266, Feb 1996.

[109] L.P. Gor'Kov and G.M. Eliashberg. Generalization of the Ginzburg-Landau equations for non-stationary problems in the case of alloys with paramagnetic impurities. Soviet Journal of Experimental and Theoretical Physics, 27:328, Feb 1968.

[110] LF Chibotaru and Vu Hung Dao. Stable fractional flux vortices in mesoscopic superconductors. Physical Review B, 81(2):020502, 2010.

[111] T. Winiecki and C.S. Adams. A fast semi-implicit finite-difference method for the tdgl equations. Journal of Computational Physics, 179(1):127-139, Jun 2002. 
[112] V.V. Shmidt and G.S. Mkrtchyan. Vortices in type-II superconductors. PhysicsUspekhi, 17(2):170-185, Dec 1974.

[113] Joseph Lee Rodgers and W Alan Nicewander. Thirteen ways to look at the correlation coefficient. The American Statistician, 42(1):59-66, 1988.

[114] LF Chibotaru and Vu Hung Dao. Stable fractional flux vortices in mesoscopic superconductors. Phys. Rev. B, 81(2):020502, Jan 2010.

[115] A.I. Larkin and Y.U.N. Ovchinnikov. Nonlinear conductivity of superconductors in the mixed state. Sov. Phys. JETP, 41(5):960-965, May 1975.

[116] A. Gurevich and V.M. Vinokur. Interband phase modes and nonequilibrium soliton structures in two-gap superconductors. Physical Rev. Lett., 90(4):047004, Jan 2003.

[117] Paul C. Canfield and George W. Crabtree. Magnesium diboride: better late than never. Physics Today, 56(3):34-40, Mar 2003.

[118] Johnpierre Paglione and Richard L. Greene. High-temperature superconductivity in iron-based materials. Nature Physics, 6(9):645-658, Sep 2010.

[119] Y. Lubashevsky, E. Lahoud, K. Chashka, D. Podolsky, and A. Kanigel. Shallow pockets and very strong coupling superconductivity in fesexte1-x. Nature Physics, 8(4):309-312, Mar 2012.

[120] M. Zehetmayer. A review of two-band superconductivity: materials and effects on the thermodynamic and reversible mixed-state properties. Superconductor Science and Technology, 26(4):043001, Feb 2013.

[121] G. Blumberg, A. Mialitsin, B.S. Dennis, M.V. Klein, N.D. Zhigadlo, and J. Karpinski. Observation of Leggetts collective mode in a multiband $M g B_{2}$ superconductor. Phys. Rev. Lett., 99(22):227002, Nov 2007.

[122] Valentin Stanev and Zlatko Tešanović. Three-band superconductivity and the order parameter that breaks time-reversal symmetry. Phys. Rev. B, 81(13):134522, Apr 2010.

[123] Julien Garaud, Johan Carlström, and Egor Babaev. Topological solitons in threeband superconductors with broken time reversal symmetry. Phys. Rev. Lett., 107(19):197001, Nov 2011.

[124] Shi-Zeng Lin and Xiao Hu. Phase solitons in multi-band superconductors with and without time-reversal symmetry. New Journal of Physics, 14(6):063021, Jun 2012. 
[125] Julien Garaud and Egor Babaev. Domain walls and their experimental signatures in s+ i s superconductors. Phys. Rev. Lett., 112(1):017003, Jan 2014.

[126] Xiao Hu and Zhi Wang. Stability and josephson effect of time-reversal-symmetrybroken multicomponent superconductivity induced by frustrated intercomponent coupling. Phys.Rev. B, 85(6):064516, Feb 2012.

[127] Zhao Huang and Xiao Hu. Fractional flux plateau in magnetization curve of multicomponent superconductor loop. Phys. Rev. B, 92(21):214516, Dec 2015.

[128] Shi-Zeng Lin and Xiao Hu. Phase solitons in multi-band superconductors with and without time-reversal symmetry. New Journal of Physics, 14(6):063021, Jun 2012.

[129] B. Maiorov, S.A. Baily, H. Zhou, O. Ugurlu, J.A. Kennison, P.C. Dowden, T.G. Holesinger, S.R. Foltyn, and L. Civale. Synergetic combination of different types of defect to optimize pinning landscape using $\mathrm{BaZrO}_{3}$-doped $\mathrm{YBa}_{2} \mathrm{Cu}_{3} \mathrm{O}_{7}$. Nature Materials, 8(5):398, May 2009.

[130] Peidong Yang and Charles M Lieber. Nanostructured high-temperature superconductors: Creation of strong-pinning columnar defects in nanorod/superconductor composites. Journal of materials research, 12(11):2981-2996, Jan 1997.

[131] Victor Moshchalkov, Roger Woerdenweber, and Wolfgang Lang. Nanoscience and engineering in superconductivity. Springer Science \& Business Media, 2010.

[132] R.B.G Kramer, A.V. Silhanek, W Gillijns, and V.V. Moshchalkov. Imaging the statics and dynamics of superconducting vortices and antivortices induced by magnetic microdisks. Phys. Rev. X, 1(2):021004, 2011.

[133] MM Salomaa and GE Volovik. Half-quantum vortices in superfluid he 3-a. Physical review letters, 55(11):1184, 1985.

[134] Anthony J Leggett. A theoretical description of the new phases of liquid he 3 . Reviews of Modern Physics, 47(2):331, 1975.

[135] Manfred Sigrist and Kazuo Ueda. Phenomenological theory of unconventional superconductivity. Reviews of Modern physics, 63(2):239, 1991.

[136] Suk Bum Chung, Hendrik Bluhm, and Eun-Ah Kim. Stability of half-quantum vortices in p x+ i p y superconductors. Physical review letters, 99(19):197002, 2007.

[137] Vadim B Geshkenbein, Anatoly I Larkin, and Antonio Barone. Vortices with half magnetic flux quanta in heavy-fermion superconductors. Physical Review B, $36(1): 235,1987$. 
[138] Hans Hilgenkamp, Henk-Jan H Smilde, Dave HA Blank, Guus Rijnders, Horst Rogalla, John R Kirtley, Chang C Tsuei, et al. Ordering and manipulation of the magnetic moments in large-scale superconducting $\pi$-loop arrays. Nature, 422(6927):50$53,2003$.

[139] John Bardeen. Quantization of flux in a superconducting cylinder. Physical Review Letters, 7(5):162, 1961.

[140] AK Geim, SV Dubonos, IV Grigorieva, KS Novoselov, FM Peeters, and VA Schweigert. Non-quantized penetration of magnetic field in the vortex state of superconductors. Nature, 407(6800):55-57, 2000.

[141] Jorge Berger and Jacob Rubinstein. Connectivity and superconductivity, volume 62. Springer Science \& Business Media, 2001. 